\title{
Pd-Catalyzed Alkyl to Aryl Migration and Cyclization: An Efficient Synthesis of Fused Polycycles via Multiple C-H Activation
}

Qinhua Huang, Alessia Fazio, Guangxiu Dai, Marino A. Campo and Richard C. Larock* Department of Chemistry, Iowa State University, Ames, IA 50011 larock@iastate.edu

General procedures. All ${ }^{1} \mathrm{H}$ and ${ }^{13} \mathrm{C}$ NMR spectra were recorded at 300 and 75.5 MHz or 400 and $100 \mathrm{MHz}$ respectively. Thin-layer chromatography was performed using commercially prepared 60-mesh silica gel plates (Whatman K6F), and visualization was effected with short wavelength UV light ( $254 \mathrm{~nm}$ ), a basic $\mathrm{KMnO}_{4}$ solution [3 $\mathrm{g}$ of $\mathrm{KMnO}_{4}+20 \mathrm{~g}$ of $\mathrm{K}_{2} \mathrm{CO}_{3}+5 \mathrm{~mL}$ of $\mathrm{NaOH}(5 \%)+300 \mathrm{~mL}$ of $\left.\mathrm{H}_{2} \mathrm{O}\right]$ and/or an acidic $p-$ anisaldehyde stain $\left[657 \mathrm{~mL}\right.$ of EtOH $(99 \%)+25 \mathrm{~mL}$ of conc. $\mathrm{H}_{2} \mathrm{SO}_{4}+7.5 \mathrm{~mL}$ of glacial $\mathrm{CH}_{3} \mathrm{CO}_{2} \mathrm{H}+10.5 \mathrm{~mL}$ of $p$-anisaldehyde]. High resolution mass spectra were recorded on a Kratos MS50TC double focusing magnetic sector mass spectrometer using EI at $70 \mathrm{eV}$. 2-Iodo-5-methoxyphenol, ${ }^{1}$ 2-iodo-5-nitrophenol, ${ }^{2}$ 2,5-diiodo-1,4-dihydroquinone ${ }^{3}$ and compound $\mathbf{2 2}^{4}$ were prepared according to literature procedures.
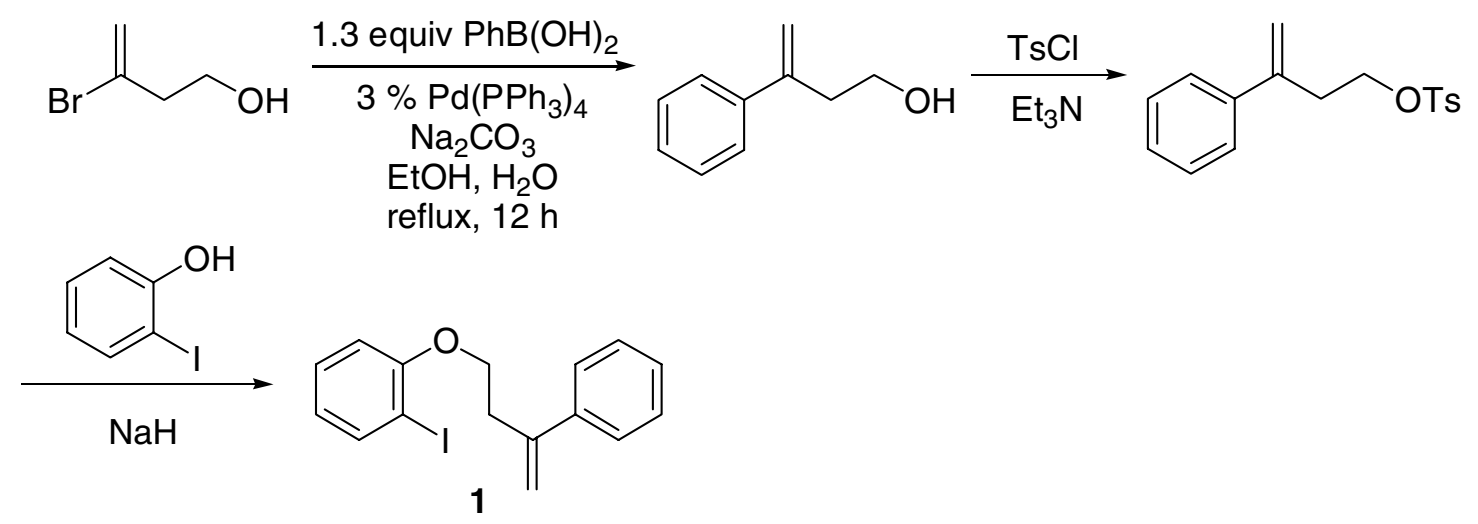

3-Phenyl-3-buten-1-ol. This compound was prepared by a modified literature procedure. ${ }^{5}$ To a stirred mixture of $\mathrm{Pd}\left(\mathrm{PPh}_{3}\right)_{4}(0.346 \mathrm{~g}, 0.3 \mathrm{mmol}), \mathrm{Na}_{2} \mathrm{CO}_{3}(2 \mathrm{M}$ aq 
solution, $10 \mathrm{~mL}, 20 \mathrm{mmol})$, benzene $(20 \mathrm{~mL})$ and 3-bromo-3-buten-1-ol $(1.51 \mathrm{~g}, 10 \mathrm{mmol})$ was added dropwise a clear solution of $\mathrm{PhB}(\mathrm{OH})_{2}(1.57 \mathrm{~g}, 13 \mathrm{mmol})$ in $\mathrm{EtOH}(10 \mathrm{~mL})$ at $25{ }^{\circ} \mathrm{C}$. The resulting mixture was then heated to reflux under an $\mathrm{Ar}$ atmosphere (oil bath temperature is $\left.70{ }^{\circ} \mathrm{C}\right)$. After $12 \mathrm{~h}$, the mixture was cooled to $25^{\circ} \mathrm{C}$ and $1.5 \mathrm{~mL}$ of $30 \%$ aq $\mathrm{H}_{2} \mathrm{O}_{2}$ was added dropwise. The resulting mixture was stirred at $25^{\circ} \mathrm{C}$ for $30 \mathrm{~min}$, diluted with $\mathrm{Et}_{2} \mathrm{O}(30 \mathrm{~mL})$ and washed with brine $(50 \mathrm{~mL})$. The organic layer was dried over $\mathrm{Na}_{2} \mathrm{SO}_{4}$, concentrated, and the residue was chromatographed (2:1 hexane/EtOAc) to afford $1.16 \mathrm{~g}$ of the indicated compound $\left(78 \%\right.$ yield) as a yellow oil: ${ }^{1} \mathrm{H} \mathrm{NMR}\left(\mathrm{CDCl}_{3}\right)$ $\delta 1.42(\mathrm{~s}, 1 \mathrm{H}), 2.80(\mathrm{t}, J=6.4 \mathrm{~Hz}, 2 \mathrm{H}), 3.73(\mathrm{t}, J=6.4 \mathrm{~Hz}, 2 \mathrm{H}), 5.17(\mathrm{~d}, J=0.8 \mathrm{~Hz}, 1 \mathrm{H})$, $5.42(\mathrm{~d}, J=0.8 \mathrm{~Hz}, 1 \mathrm{H}), 7.26-7.36(\mathrm{~m}, 3 \mathrm{H}), 7.41-7.43(\mathrm{~m}, 2 \mathrm{H})$.

3-Phenyl-3-butenyl tosylate. To a solution of TsCl (0.96 g, $5.05 \mathrm{mmol})$ in $\mathrm{CH}_{2} \mathrm{Cl}_{2}$ $(10 \mathrm{~mL})$ was added dropwise a solution of 3-phenyl-3-buten-1-ol $(0.74 \mathrm{~g}, 5.0 \mathrm{mmol})$ in $\mathrm{CH}_{2} \mathrm{Cl}_{2}(5 \mathrm{~mL})$ at $0{ }^{\circ} \mathrm{C}$ under an $\mathrm{Ar}$ atmosphere. $\mathrm{Et}_{3} \mathrm{~N}(0.7 \mathrm{~mL}, 5.05 \mathrm{~mol})$ was added slowly and the reaction mixture was stirred at $25{ }^{\circ} \mathrm{C}$ until the reaction reached completion as indicated by thin layer chromatography. The reaction mixture was diluted with $\mathrm{Et}_{2} \mathrm{O}$ (20 mL), washed with brine $(30 \mathrm{~mL})$, and the organic layer was dried over $\mathrm{Na}_{2} \mathrm{SO}_{4}$. The solvent was removed under reduced pressure and the residue was chromatographed (5:1 hexane/EtOAc) to afford $1.23 \mathrm{~g}$ of the indicated compound ( $82 \%$ yield) as a brown oil: ${ }^{1} \mathrm{H} \mathrm{NMR}\left(\mathrm{CDCl}_{3}\right) \delta 2.44(\mathrm{~s}, 3 \mathrm{H}), 2.86(\mathrm{dt}, J=0.8,7.2 \mathrm{~Hz}, 2 \mathrm{H}), 4.10(\mathrm{t}, J=7.2 \mathrm{~Hz}, 2 \mathrm{H})$, $5.08(\mathrm{~s}, 1 \mathrm{H}), 5.34(\mathrm{~s}, 1 \mathrm{H}), 7.25-7.31(\mathrm{~m}, 7 \mathrm{H}), 7.72-7.74(\mathrm{~m}, 2 \mathrm{H}) ;{ }^{13} \mathrm{C} \mathrm{NMR}\left(\mathrm{CDCl}_{3}\right) \delta$ 21.8, 35.0, 68.9, 115.5, 126.2, 128.0, 128.1, 128.7, 130.0, 133.3, 139.9, 143.0, 144.9. 2-Iodophenyl 3-phenyl-3-butenyl ether (1). To a suspension of NaH (60\% in mineral oil, $44 \mathrm{mg}, 1.12 \mathrm{mmol})$ in dry $\mathrm{DMF}(1.5 \mathrm{~mL})$ was added a solution of 2- 
iodophenol $(0.23 \mathrm{~g}, 1.02 \mathrm{mmol})$ in dry $\operatorname{DMF}(2.0 \mathrm{~mL})$ at $0{ }^{\circ} \mathrm{C}$ under an $\mathrm{Ar}$ atmosphere. Lots of bubbles were generated. After $20 \mathrm{~min}$, a solution of 3-phenyl-3-butenyl tosylate $(0.28 \mathrm{~g}, 0.93 \mathrm{mmol})$ in dry DMF $(2.0 \mathrm{~mL})$ was added slowly. The reaction mixture was allowed to warm to and stirred at $25{ }^{\circ} \mathrm{C}$ for $1 \mathrm{~d}$, diluted with $\mathrm{Et}_{2} \mathrm{O}(20 \mathrm{~mL})$, and washed with brine $(20 \mathrm{~mL})$. The organic layer was dried over $\mathrm{Na}_{2} \mathrm{SO}_{4}$, filtered, and concentrated under reduce pressure. The residue was chromatographed (35:1 hexane/EtOAc) to afford $0.15 \mathrm{~g}$ of the indicated compound (46\% yield) as a yellow oil: ${ }^{1} \mathrm{H} \mathrm{NMR}\left(\mathrm{CDCl}_{3}\right) \delta 3.07$ $(\mathrm{dt}, J=0.9,6.9 \mathrm{~Hz}, 2 \mathrm{H}), 4.10(\mathrm{t}, J=6.9 \mathrm{~Hz}, 2 \mathrm{H}), 5.25-5.27(\mathrm{~m}, 1 \mathrm{H}), 5.43(\mathrm{~d}, J=1.2 \mathrm{~Hz}$, 1H), 6.65-6.71 (m, 1H), $6.73(\mathrm{dd}, J=1.2,8.4 \mathrm{~Hz}, 1 \mathrm{H}), 7.21-7.37(\mathrm{~m}, 4 \mathrm{H}), 7.45-7.48(\mathrm{~m}$, 2H), $7.76(\mathrm{dd}, J=2.0,8.4 \mathrm{~Hz}, 1 \mathrm{H}) ;{ }^{13} \mathrm{C} \mathrm{NMR}\left(\mathrm{CDCl}_{3}\right) \delta 35.3,68.2,86.9,112.5,115.2$, 122.7, 126.4, 127.9, 128.7, 129.6, 139.7, 140.8, 144.5, 157.6; IR (neat, $\mathrm{cm}^{-1}$ ) 3080, 3056, 2946, 1581, 1464; HRMS Calcd for $\mathrm{C}_{16} \mathrm{H}_{15} \mathrm{IO}: 350.0168$. Found: 350.0171.

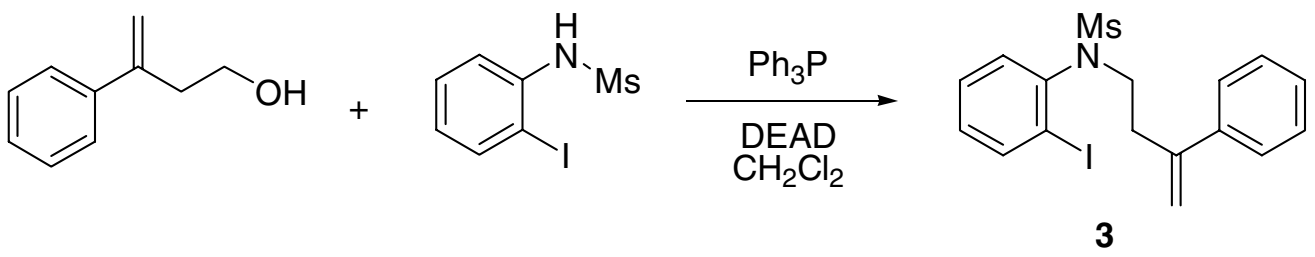

$N$-(2-Iodophenyl)- $N$-(3-phenyl-3-butenyl)methanesulfonamide (3). To a stirred mixture of 3-phenyl-3-buten-1-ol (0.296 g, $1.3 \mathrm{mmol}), \mathrm{PPh}_{3}(0.34 \mathrm{~g}, 1.3 \mathrm{mmol}), \mathrm{N}-(2-$ iodophenyl)methanesulfonamide $(0.297 \mathrm{~g}, 1.0 \mathrm{mmol})$ and $\mathrm{CH}_{2} \mathrm{Cl}_{2}(10 \mathrm{~mL})$ was added slowly a solution of diethyl azodicarboxylate (DEAD) $(0.20 \mathrm{~mL}, 1.3 \mathrm{mmol})$ in $\mathrm{CH}_{2} \mathrm{Cl}_{2}(2$ $\mathrm{mL}$ ) at $0{ }^{\circ} \mathrm{C}$ under an $\mathrm{Ar}$ atmosphere. The resulting yellow solution was stirred at $0{ }^{\circ} \mathrm{C}$ for $2 \mathrm{~h}$, diluted with $\mathrm{Et}_{2} \mathrm{O}(20 \mathrm{~mL})$, and washed with brine $(20 \mathrm{~mL})$. The organic layer was dried over $\mathrm{Na}_{2} \mathrm{SO}_{4}$ and the solvent was removed under reduced pressure. The 
residue was chromatographed (3:1 hexane/EtOAc) to afford $0.14 \mathrm{~g}$ of the indicated compound (34 \% yield) as a colorless oil: ${ }^{1} \mathrm{H}$ NMR $\left(\mathrm{CDCl}_{3}\right) \delta$ 2.77-2.92 (m, 2H), $3.04(\mathrm{~s}$, 3H), 3.59-3.68 (m, 1H), 3.79-3.88 (m, 1H), $5.07(\mathrm{~s}, 1 \mathrm{H}), 5.35(\mathrm{~s}, 1 \mathrm{H}), 7.07-7.12(\mathrm{~m}, 1 \mathrm{H})$, 7.25-7.33 (m, 5H), 7.40-7.44 (m, 1H), $7.47(\mathrm{dd}, J=1.6,7.6 \mathrm{~Hz}, 1 \mathrm{H}), 7.94-7.96(\mathrm{~m}, 1 \mathrm{H})$; ${ }^{13} \mathrm{C} \mathrm{NMR}\left(\mathrm{CDCl}_{3}\right) \delta 34.8,41.0,50.9,101.6,114.5,126.1,128.0,128.7,129.5,130.4$, 132.7, 140.0, 140.7, 141.2, 144.7; IR $\left(\mathrm{CDCl}_{3}, \mathrm{~cm}^{-1}\right)$ 3081, 3055, 3023, 2932, 1763, 1721, 1626, 1466, 1340; HRMS Calcd for $\mathrm{C}_{17} \mathrm{H}_{18} \mathrm{INO}_{2} \mathrm{~S}$ : 427.0103. Found: 427.0114.
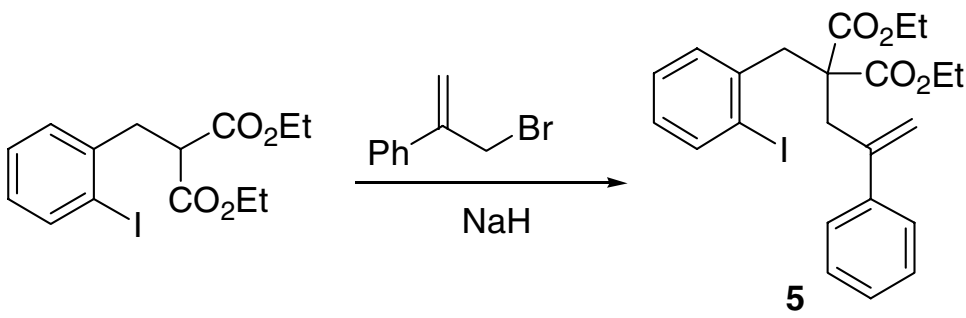

Diethyl 2-(2-iodobenzyl)-2-(2-phenylallyl)malonate (5). Using the procedure used to prepare compound $\mathbf{1}$, but employing diethyl 2-(2-iodobenzyl)malonate and 2phenylallyl bromide, afforded compound 5 in an $82 \%$ yield (19:1 hexane/EtOAc) as a yellow oil: ${ }^{1} \mathrm{H} \mathrm{NMR}\left(\mathrm{CDCl}_{3}\right) \delta 1.05(\mathrm{t}, J=7.2 \mathrm{~Hz}, 6 \mathrm{H}), 3.33(\mathrm{~s}, 2 \mathrm{H}), 3.40(\mathrm{~s}, 2 \mathrm{H}), 3.76-$ $3.90(\mathrm{~m}, 4 \mathrm{H}), 5.80(\mathrm{~d}, J=1.2 \mathrm{~Hz}, 1 \mathrm{H}), 5.28(\mathrm{~d}, J=1.2 \mathrm{~Hz}, 1 \mathrm{H}), 6.81-6.87(\mathrm{~m}, 1 \mathrm{H}), 7.17-$ $7.36(\mathrm{~m}, 7 \mathrm{H}), 7.79(\mathrm{dd}, J=1.2,8.1 \mathrm{~Hz}, 1 \mathrm{H}) ;{ }^{13} \mathrm{C} \mathrm{NMR}\left(\mathrm{CDCl}_{3}\right) \delta 13.9,39.6,43.0,58.3$, 61.5, 103.4, 118.7, 127.0, 127.7, 128.1, 128.2, 128.3, 130.0, 139.8, 140.5, 141.7, 144.4, 170.7; IR $\left(\mathrm{CDCl}_{3}, \mathrm{~cm}^{-1}\right) 3057,2981,1728,1626,1468,1444$; HRMS Calcd for $\mathrm{C}_{23} \mathrm{H}_{25} \mathrm{IO}_{4}$ : 492.0798. Found: 492.0809 . 


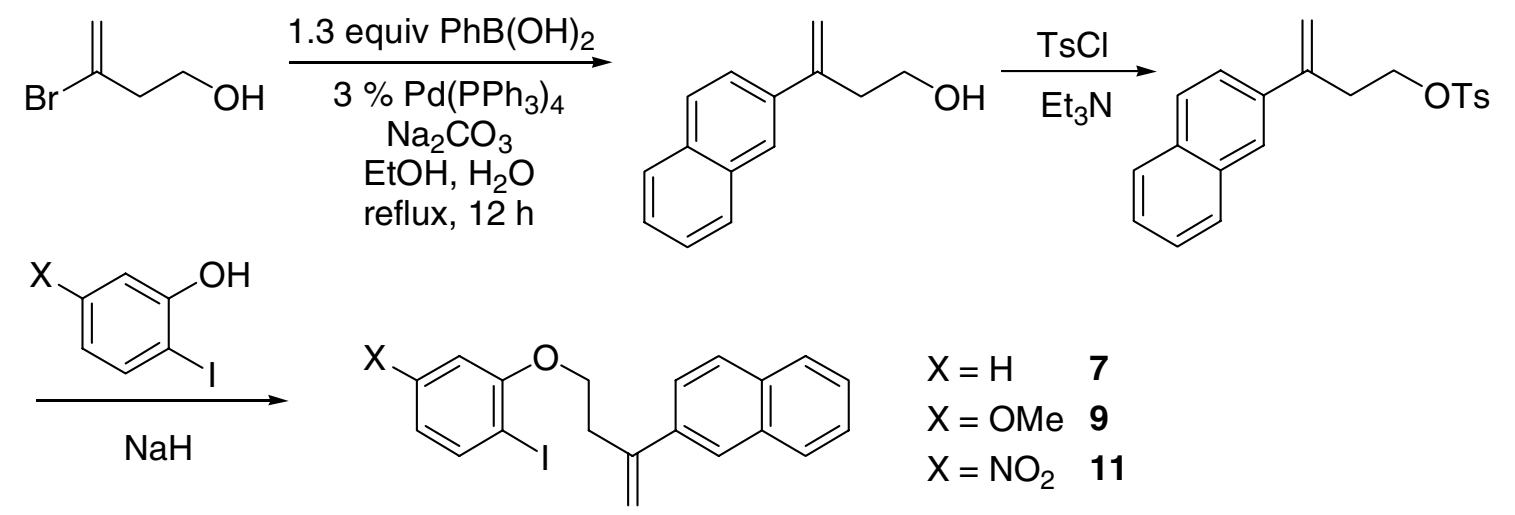

3-(2-Naphthyl)-3-buten-1-ol. Using the procedure used to prepare 3-phenyl-3buten-1-ol, but employing 3-bromo-3-buten-1-ol and 2-naphthylboronic acid, afforded the indicated compound in a $76 \%$ yield (4:1 hexane/EtOAc) as a yellow solid: $\mathrm{mp} 45-46$ ${ }^{\circ} \mathrm{C} ;{ }^{1} \mathrm{H}$ NMR $\left(\mathrm{CDCl}_{3}\right) \delta 1.58(\mathrm{br} \mathrm{s}, 1 \mathrm{H}), 2.92(\mathrm{dt}, J=0.9,6.4 \mathrm{~Hz}, 2 \mathrm{H}), 3.79(\mathrm{t}, J=6.4 \mathrm{~Hz}$, 2H), $5.28(\mathrm{~d}, J=1.0 \mathrm{~Hz}, 1 \mathrm{H}), 5.57(\mathrm{~d}, J=1.0 \mathrm{~Hz}, 1 \mathrm{H}), 7.45-7.52(\mathrm{~m}, 2 \mathrm{H}), 7.59(\mathrm{dd}, J=$ 1.7, 8.7 Hz, 1H), 7.80-7.85 (m, 4H); ${ }^{13} \mathrm{C} \mathrm{NMR}\left(\mathrm{CDCl}_{3}\right)$ 38.8, 61.3, 115.4, 124.7, 125.1, $126.3,126.5,127.8,128.3,128.4,133.1,133.6,137.8,144.8$.

3-(2-Naphthyl)-3-butenyl tosylate. Using the procedure used to prepare 3-phenyl-3butenyl tosylate, but employing 3-(2-naphthyl)-3-buten-1-ol, afforded the indicated compound in a $60 \%$ yield $\left(7: 3\right.$ hexane/EtOAc) as a yellow oil: ${ }^{1} \mathrm{H}$ NMR $\left(\mathrm{CDCl}_{3}\right) \delta 2.35$ (s, 3H), $2.98(\mathrm{dt}, J=0.9,6.9 \mathrm{~Hz}, 2 \mathrm{H}), 4.15(\mathrm{t}, J=6.9 \mathrm{~Hz}, 2 \mathrm{H}), 5.19(\mathrm{~d}, J=0.9 \mathrm{~Hz}, 1 \mathrm{H})$, $5.49(\mathrm{~s}, 1 \mathrm{H}), 7.17-7.20(\mathrm{~m}, 2 \mathrm{H}), 7.42-7.48(\mathrm{~m}, 3 \mathrm{H}), 7.66-7.69(\mathrm{~m}, 3 \mathrm{H}), 7.73-7.78(\mathrm{~m}, 3 \mathrm{H})$;

${ }^{13} \mathrm{C} \mathrm{NMR}\left(\mathrm{CDCl}_{3}\right) \delta 21.7,35.0,68.9,116.1,124.4,124.9,126.3,126.5,127.2,127.7$, $128.0,128.3,128.3,129.9,133.1,133.4,137.0,142.8,144.8$.

2-Iodophenyl 3-(2-naphthyl)-3-butenyl ether (7). Using the procedure used to prepare compound 1, but employing 2-iodophenol and 3-(2-naphthyl)-3-butenyl tosylate, afforded the indicated compound in a $58 \%$ yield (30:1 hexane/EtOAc) as a colorless oil: 
${ }^{1} \mathrm{H} \mathrm{NMR}\left(\mathrm{CDCl}_{3}\right) \delta 3.18(\mathrm{t}, J=6.9 \mathrm{~Hz}, 2 \mathrm{H}), 4.14(\mathrm{t}, J=6.9 \mathrm{~Hz}, 2 \mathrm{H}), 5.36(\mathrm{~s}, 1 \mathrm{H}), 5.57(\mathrm{~s}$, 1H), 6.64-6.72 (m, 2H), 7.18-7.23 (m, 1H), 7.44-7.48 (m, 2H), $7.62(\mathrm{dd}, J=1.5,8.4 \mathrm{~Hz}$, 1H), 7.73-7.88 (m, 5H); ${ }^{13} \mathrm{C} \mathrm{NMR}\left(\mathrm{CDCl}_{3}\right)$ 35.3, 68.3, 86.9, 112.5, 115.7, 122.7, 124.8, 125.0, 126.2, 126.4, 127.7, 128.2, 128.4, 129.6, 133.1, 133.6, 137.9, 139.7, 144.3, 157.6; IR $\left(\mathrm{CHCl}_{3}, \mathrm{~cm}^{-1}\right)$ 3056, 3009, 2931, 1581, 1477, 1459; HRMS Calcd for $\mathrm{C}_{20} \mathrm{H}_{17} \mathrm{IO}$ : 400.0324. Found: 400.0332 .

2-Iodo-5-methoxyphenyl 3-(2-naphthyl)-3-butenyl ether (9). Using the procedure used to prepare compound $\mathbf{1}$, but employing 2-iodo-5-methoxyphenol ${ }^{1}$ and 3-(2naphthyl)-3-butenyl tosylate, afforded the indicated compound in a $56 \%$ yield (24:1 hexane/EtOAc) as a white solid: $\mathrm{mp} 72-73{ }^{\circ} \mathrm{C} ;{ }^{1} \mathrm{H} \mathrm{NMR}\left(\mathrm{CDCl}_{3}\right) \delta 3.18(\mathrm{t}, J=7.2 \mathrm{~Hz}$, 2H), $3.70(\mathrm{~s}, 3 \mathrm{H}), 4.11(\mathrm{t}, J=7.2 \mathrm{~Hz}, 2 \mathrm{H}), 5.36(\mathrm{~s}, 1 \mathrm{H}), 5.57(\mathrm{~s}, 1 \mathrm{H}), 6.28(\mathrm{dd}, J=1.6$, $8.4 \mathrm{~Hz}, 1 \mathrm{H}), 6.31(\mathrm{~d}, J=2.8 \mathrm{~Hz}, 1 \mathrm{H}), 7.42-7.49(\mathrm{~m}, 2 \mathrm{H}), 7.59(\mathrm{~d}, J=8.8 \mathrm{~Hz}, 1 \mathrm{H}), 7.62$ $(\mathrm{dd}, J=1.6,8.8 \mathrm{~Hz}, 1 \mathrm{H}), 7.79-7.84(\mathrm{~m}, 3 \mathrm{H}), 7.88(\mathrm{~s}, 1 \mathrm{H}) ;{ }^{13} \mathrm{C} \mathrm{NMR}\left(\mathrm{CDCl}_{3}\right)$ 35.1, 55.5, 68.1, 75.5, 100.2, 107.4, 115.6, 124.6, 124.9, 126.1, 126.3, 127.6, 128.1, 128.3, 132.9, 133.4, 137.7, 139.2, 144.0, 158.2, 161.3; IR $\left(\mathrm{CHCl}_{3}, \mathrm{~cm}^{-1}\right) 3057,3013,2937,1577,1483$, 1304; HRMS Calcd for $\mathrm{C}_{21} \mathrm{H}_{19} \mathrm{IO}_{2}$ : 430.0430. Found: 430.0435 .

2-Iodo-5-nitrophenyl 3-(2-naphthyl)-3-butenyl ether (11). Using the procedure used to prepare compound 1, but employing 2-iodo-5-nitrophenol ${ }^{2}$ and 3-(2-naphthyl)-3butenyl tosylate, afforded the indicated compound in a $30 \%$ yield (35:1 hexane/EtOAc) as a pale yellow solid: $\mathrm{mp} 94-96{ }^{\circ} \mathrm{C} ;{ }^{1} \mathrm{H} \mathrm{NMR}\left(\mathrm{CDCl}_{3}\right) \delta 3.23(\mathrm{t}, J=6.6 \mathrm{~Hz}, 2 \mathrm{H}), 4.24(\mathrm{t}$, $J=6.6 \mathrm{~Hz}, 2 \mathrm{H}), 5.39(\mathrm{~d}, J=0.6 \mathrm{~Hz}, 1 \mathrm{H}), 5.60(\mathrm{~s}, 1 \mathrm{H}), 7.43-7.56(\mathrm{~m}, 4 \mathrm{H}), 7.62(\mathrm{dd}, J=$ 1.8, 9.7 Hz, 1H), 7.81-7.94 (m, $5 \mathrm{H}),{ }^{13} \mathrm{C} \mathrm{NMR}\left(\mathrm{CDCl}_{3}\right) \delta 35.1,68.9,95.7,106.2,116.2$, 117.0, 124.7, 125.1, 126.3, 126.6, 127.8, 128.4, 128.4, 133.1, 133.6, 137.6, 139.9, 143.9, 
149.3, 158.2; IR $\left(\mathrm{CHCl}_{3}, \mathrm{~cm}^{-1}\right) 3018,2927,1569,1525,1349$; HRMS Calcd for $\mathrm{C}_{20} \mathrm{H}_{16} \mathrm{INO}_{5}$ : 445.0175. Found: 445.0186.<smiles>COCOc1cccc2ccccc12</smiles><smiles>[R]C(C)(C)Br</smiles><smiles>COCOc1c(I)ccc2ccccc12</smiles>

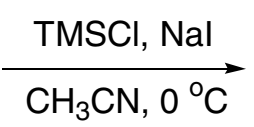<smiles>Oc1c(I)ccc2ccccc12</smiles>

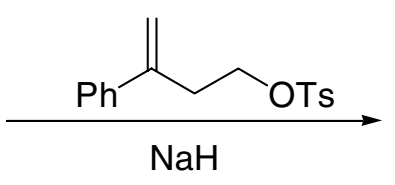<smiles>C=C(CCOc1c(I)ccc2ccccc12)c1ccccc1</smiles>

2-Iodo-1-(methoxymethyloxy)naphthalene. To a stirred solution of 1(methoxymethyloxy)naphthalene (1.75 g, $9.3 \mathrm{mmol})$ in dry THF $(45 \mathrm{~mL})$ was added $n$ $\operatorname{BuLi}(2.5 \mathrm{M}$ in hexane, $4.5 \mathrm{~mL}, 11.2 \mathrm{mmol})$ at $-78{ }^{\circ} \mathrm{C}$ under an $\mathrm{Ar}$ atmosphere. The reaction mixture was then warmed to and stirred at $25{ }^{\circ} \mathrm{C}$. After $30 \mathrm{~min}$, the resulting deep orange solution was cooled to $-78{ }^{\circ} \mathrm{C}$ and a solution of $\mathrm{I}_{2}(4.0 \mathrm{~g}, 11.2 \mathrm{mmol})$ in dry THF $(20 \mathrm{~mL})$ was added dropwise. The resulting deep blue solution was stirred at $-78{ }^{\circ} \mathrm{C}$ for another $10 \mathrm{~min}$ and then warmed to $25{ }^{\circ} \mathrm{C}$. The reaction mixture was diluted with $\mathrm{Et}_{2} \mathrm{O}(30 \mathrm{~mL})$ and excess $\mathrm{I}_{2}$ was destroyed by satd aq $\mathrm{Na}_{2} \mathrm{~S}_{2} \mathrm{O}_{3}(30 \mathrm{~mL})$. The organic layer was dried over $\mathrm{Na}_{2} \mathrm{SO}_{4}$ and the solvent was removed under reduced pressure. The residue was chromatographed (19:1 hexane/EtOAc) on silica gel to afford $2.0 \mathrm{~g}$ of the indicated compound in a $68 \%$ yield as a yellow oil: ${ }^{1} \mathrm{H}$ NMR $\left(\mathrm{CDCl}_{3}\right) \delta 3.76(\mathrm{~s}, 3 \mathrm{H})$, $5.25(\mathrm{~s}, 2 \mathrm{H}), 7.39(\mathrm{~s}, J=8.7 \mathrm{~Hz}, 1 \mathrm{H}), 7.48-7.54(\mathrm{~m}, 2 \mathrm{H}), 7.79(\mathrm{~d}, J=8.7 \mathrm{~Hz}, 1 \mathrm{H}), 7.81-$ $7.83(\mathrm{~m}, 1 \mathrm{H}), 8.15-8.19(\mathrm{~m}, 1 \mathrm{H})$. 
2-Iodo-1-naphthol. To a stirred mixture of NaI (0.91 mg, 6.0 mmol), 2-iodo-1(methoxymethyloxy)naphthalene $(0.96 \mathrm{~g}, 3.0 \mathrm{mmol})$ and $\mathrm{CH}_{3} \mathrm{CN}(30 \mathrm{~mL})$ was added dropwise $\mathrm{TMSCl}(0.57 \mathrm{~mL}, 4.5 \mathrm{mmol})$ at $0{ }^{\circ} \mathrm{C}$ under an $\mathrm{Ar}$ atmosphere. The mixture was stirred at $0{ }^{\circ} \mathrm{C}$ for $10 \mathrm{~min}$ and then warmed to $25^{\circ} \mathrm{C}$. As the reaction proceeded, the reaction mixture became more and more cloudy. After $2 \mathrm{~h}$, the reaction was diluted with $\mathrm{Et}_{2} \mathrm{O}(30 \mathrm{~mL})$ and washed with satd aq $\mathrm{Na}_{2} \mathrm{~S}_{2} \mathrm{O}_{3}(30 \mathrm{~mL})$. The organic layer was dried over $\mathrm{Na}_{2} \mathrm{SO}_{4}$, filtered, and the solvent was removed under reduced pressure. The residue was chromatographed (4:1 hexane/EtOAc) on silica gel to afford $0.35 \mathrm{~g}$ of the indicated compound in a $43 \%$ yield as a yellow solid: $\mathrm{mp} 54-55{ }^{\circ} \mathrm{C} ;{ }^{1} \mathrm{H}$ NMR $\left(\mathrm{CDCl}_{3}\right) \delta 5.72(\mathrm{~s}$, 1H), $7.20(\mathrm{~d}, J=8.7 \mathrm{~Hz}, 1 \mathrm{H}), 7.46-7.54(\mathrm{~d}, J=2 \mathrm{H}), 7.61(\mathrm{~d}, J=9.7 \mathrm{~Hz}, 1 \mathrm{H}), 7.75-7.79$ (m, $1 \mathrm{H}), 8.22-8.25(\mathrm{~m}, 1 \mathrm{H}) ;{ }^{13} \mathrm{C} \mathrm{NMR}\left(\mathrm{CDCl}_{3}\right) \delta 79.5,122.2,122.8,123.9,126.3,127.3$, $127.7,133.8,134.7,151.3$.

2-Iodonaphth-1-yl 3-phenyl-3-butenyl ether (13). Using the procedure used to prepare compound 1, but employing 2-iodo-1-naphthol and 3-phenyl-3-butenyl tosylate, afforded compound $\mathbf{1 3}$ in a $36 \%$ yield (49:1 hexane/EtOAc) as a white solid: mp 74-75 ${ }^{\circ} \mathrm{C} ;{ }^{1} \mathrm{H}$ NMR $\left(\mathrm{CDCl}_{3}\right) \delta 3.26(\mathrm{dt}, J=0.9,6.9 \mathrm{~Hz}, 2 \mathrm{H}), 4.15(\mathrm{t}, J=6.9 \mathrm{~Hz}, 2 \mathrm{H}), 5.31(\mathrm{~d}, J$ $=1.2 \mathrm{H}, 1 \mathrm{H}), 5.48(\mathrm{~d}, J=1.2 \mathrm{~Hz}, 1 \mathrm{H}), 7.27-7.49(\mathrm{~m}, 6 \mathrm{H}), 7.51-7.55(\mathrm{~m}, 2 \mathrm{H}), 7.74(\mathrm{~d}, J=$ 8.7 Hz, 1H), 7.77-7.80 (m, 1H), 7.91-7.94 (m, 1H); ${ }^{13} \mathrm{C} \mathrm{NMR}\left(\mathrm{CDCl}_{3}\right) \delta 36.3,73.0,87.8$, $114.9,122.4,125.8,126.4,126.7,126.9,127.9,128.1,128.7,128.7,134.9,135.4,140.7$, 144.7, 155.6; IR $\left(\mathrm{CHCl}_{3}, \mathrm{~cm}^{-1}\right)$ 3056, 3016, 2928, 1598, 1497, 1361; HRMS Calcd for $\mathrm{C}_{20} \mathrm{H}_{17} \mathrm{IO}: 400.0324$. Found: 400.0331 . 

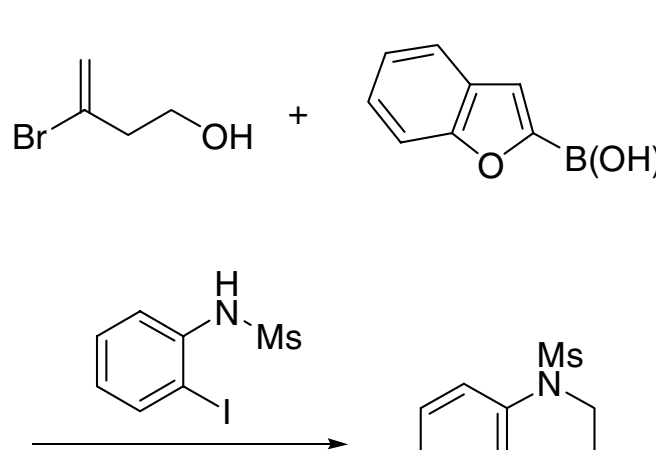

$\mathrm{Ph}_{3} \mathrm{P}, \mathrm{DEAD}$ $\mathrm{CH}_{2} \mathrm{Cl}_{2}$

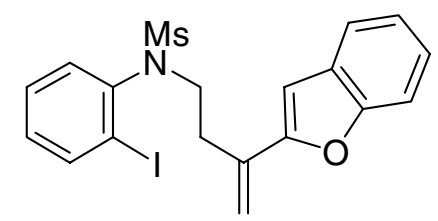

15

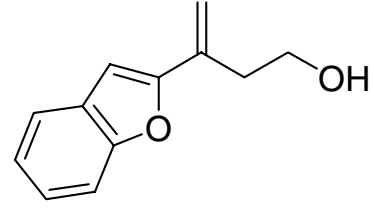

reflux

3-Benzofuran-2-yl-3-buten-1-ol. Using the procedure used to prepare 3-phenyl-3buten-1-ol, but employing benzofuran-2-boronic acid and 3-bromo-3-buten-1-ol, afforded the indicated compound in a $78 \%$ yield $\left(4: 1\right.$ hexane/EtOAc) as a yellow oil: ${ }^{1} \mathrm{H}$ NMR $\left(\mathrm{CDCl}_{3}\right) \delta 1.83(\mathrm{br} \mathrm{s}, 1 \mathrm{H}), 2.73(\mathrm{t}, J=5.6 \mathrm{~Hz}, 2 \mathrm{H}), 3.87(\mathrm{dt}, J=0.8,5.6 \mathrm{~Hz}, 2 \mathrm{H}), 5.27(\mathrm{~s}$, 1H), $5.94(\mathrm{~s}, 1 \mathrm{H}), 6.69(\mathrm{~s}, 1 \mathrm{H}), 7.19-7.22(\mathrm{~m}, 1 \mathrm{H}), 7.26-7.30(\mathrm{~m}, 1 \mathrm{H}), 7.46(\mathrm{~d}, J=8.0 \mathrm{~Hz}$, $1 \mathrm{H}), 7.53(\mathrm{~d}, J=8.0 \mathrm{~Hz}, 1 \mathrm{H}) ;{ }^{13} \mathrm{C} \mathrm{NMR}\left(\mathrm{CDCl}_{3}\right) 36.7,61.6,103.2,111.2,114.9,121.2$, 123.0, 124.9, 129.0, 134.1, 154.9, 155.9.

\section{$N$-(3-Benzofuran-2-yl-3-butenyl)- $N$-(2-iodophenyl)methanesulfonamide (15).}

Using the procedure used to prepare compound $\mathbf{3}$, but employing 3-benzofuran-2-yl-3buten-1-ol and $N$-(2-iodophenyl)methanesulfonamide, afforded the indicated compound in a $78 \%$ yield (3:1 hexane/EtOAc) as a yellow oil: ${ }^{1} \mathrm{H} \mathrm{NMR}\left(\mathrm{CDCl}_{3}\right) \delta 2.72-2.80(\mathrm{~m}$, 1H), 2.84-2.91 (m, 1H), $3.10(\mathrm{~s}, 3 \mathrm{H}), 3.70-3.74(\mathrm{~m}, 1 \mathrm{H}), 3.97-4.02(\mathrm{~m}, 1 \mathrm{H}), 5.18(\mathrm{~s}, 1 \mathrm{H})$, $5.85(\mathrm{~s}, 1 \mathrm{H}), 6.68(\mathrm{~s}, 1 \mathrm{H}), 7.11-7.15(\mathrm{~m}, 1 \mathrm{H}), 7.17-7.21(\mathrm{~m}, 1 \mathrm{H}), 7.24-7.28(\mathrm{~m}, 1 \mathrm{H}), 7.41$ $(\mathrm{dd}, J=0.4,8.0 \mathrm{~Hz}, 1 \mathrm{H}), 7.44-7.48(\mathrm{~m}, 1 \mathrm{H}), 7.52-7.54(\mathrm{~m}, 2 \mathrm{H}), 7.98(\mathrm{dd}, J=1.2,8.0 \mathrm{~Hz}$, $1 \mathrm{H}) ;{ }^{13} \mathrm{C} \mathrm{NMR}\left(\mathrm{CDCl}_{3}\right) 33.4,41.2,51.7,101.3,103.4,111.1,114.9,121.4,123.0,125.0$, 
129.0, 129.6, 130.6, 132.9, 133.9, 140.8, 141.1, 154.9, 155.4; IR (neat, $\mathrm{cm}^{-1}$ ) 3060, 2980, 1799, 1722, 1467, 1339; HRMS Calcd for $\mathrm{C}_{19} \mathrm{H}_{18} \mathrm{INO}_{3} \mathrm{~S}: 467.0052$. Found: 467.0062.<smiles>COCOc1ccc(Oc2ccccc2)cc1</smiles><smiles>C=C(C)CCOc1ccc(Oc2ccccc2)cc1I</smiles>

3-Iodo-4-(methoxymethyloxy)phenyl phenyl ether. Using the procedure used to prepare 2-iodo-1-(methoxymethyloxy)naphthalene, but employing 4(methoxymethyloxy)phenyl phenyl ether, afforded the indicated compound in a $70 \%$ yield (15:1 hexane/EtOAc) as a colorless oil: ${ }^{1} \mathrm{H}$ NMR $\left(\mathrm{CDCl}_{3}\right) \delta 3.53(\mathrm{~s}, 3 \mathrm{H}), 5.20(\mathrm{~s}$, 2H), 6.95-6.99 (m, 3H), 7.04 (d, $J=8.8 \mathrm{~Hz}, 1 \mathrm{H}), 7.07-7.11(\mathrm{~m}, 1 \mathrm{H}), 7.30-7.34(\mathrm{~m}, 2 \mathrm{H})$, $7.45(\mathrm{~d}, J=2.8 \mathrm{~Hz}, 1 \mathrm{H}) ;{ }^{13} \mathrm{C} \mathrm{NMR}\left(\mathrm{CDCl}_{3}\right) 56.7,87.5,95.8,115.9,118.4,120.4,123.4$, $130.0,130.3,152.1,152.7,157.8$.

2-Iodo-4-phenoxyphenol. Using the procedure used to prepare 2-iodo-1-naphthol, but employing 3-iodo-4-(methoxymethyloxy)phenyl phenyl ether, afforded the indicated compound in a $100 \%$ yield $\left(7: 1\right.$ hexane/EtOAc) as a yellow oil: ${ }^{1} \mathrm{H} \mathrm{NMR}\left(\mathrm{CDCl}_{3}\right) \delta$ $5.11(\mathrm{~s}, 1 \mathrm{H}), 6.93-6.97(\mathrm{~m}, 4 \mathrm{H}), 7.05-7.11(\mathrm{~m}, 1 \mathrm{H}), 7.29-7.35(\mathrm{~m}, 3 \mathrm{H}) ;{ }^{13} \mathrm{C} \mathrm{NMR}\left(\mathrm{CDCl}_{3}\right)$ $85.3,115.5,118.1,121.8,123.2,129.0,130.0,150.8,151.5,158.1$. 
2-Iodo-4-phenoxyphenyl 3-methyl-3-butenyl ether (17). Using the procedure used to prepare compound 1, but employing 2-iodo-4-phenoxyphenol and 3-methyl-3-butenyl tosylate, afforded compound 17 in a $62 \%$ yield (30:1 hexane/EtOAc) as a yellow oil: ${ }^{1} \mathrm{H}$ $\operatorname{NMR}\left(\mathrm{CDCl}_{3}\right) \delta 1.85(\mathrm{~s}, 3 \mathrm{H}), 2.57(\mathrm{t}, J=6.8 \mathrm{~Hz}, 2 \mathrm{H}), 4.10(\mathrm{t}, J=6.8 \mathrm{~Hz}, 2 \mathrm{H}), 6.78(\mathrm{~d}, J$ $=9.2 \mathrm{~Hz}, 1 \mathrm{H}), 6.93-6.96(\mathrm{~m}, 2 \mathrm{H}), 6.99(\mathrm{dd}, J=2.8,9.2 \mathrm{~Hz}, 1 \mathrm{H}), 7.05-7.09(\mathrm{~m}, 1 \mathrm{H}), 7.30-$ $7.34(\mathrm{~m}, 2 \mathrm{H}), 7.47(\mathrm{~d}, J=2.8 \mathrm{~Hz}, 1 \mathrm{H}) ;{ }^{13} \mathrm{C} \mathrm{NMR}\left(\mathrm{CDCl}_{3}\right) \delta 23.3,37.4,68.9,86.9,112.6$, $112.7,118.1,120.5,123.1,129.9,130.7,142.3,150.9,154.3,158.1$; IR (neat, $\mathrm{cm}^{-1}$ ) 3072, 2967, 1649. 1587, 1482; HRMS Calcd for $\mathrm{C}_{17} \mathrm{H}_{17} \mathrm{IO}_{2}$ : 380.0273. Found: 380.0282 .<smiles>Oc1cc(I)c(O)cc1I</smiles>
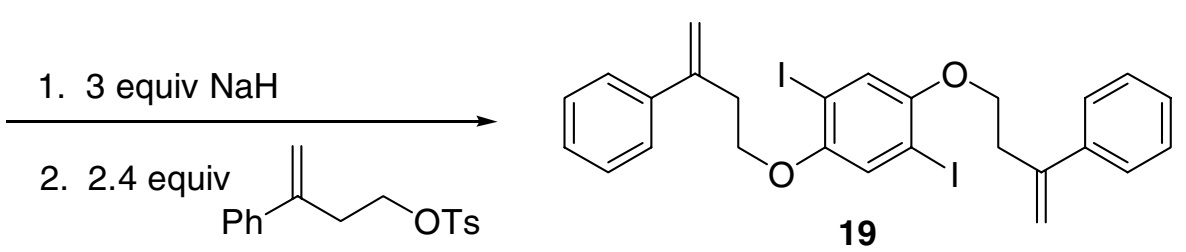

1,4-Diiodo-2,5-bis(3-phenyl-3-butenyloxy)benzene (19). Using the procedure used to prepare compound 1, but employing 2,5-diiodo-1,4-diphenol ${ }^{3}$ and 3-phenyl-3-butenyl tosylate, afforded compound 19 in a $44 \%$ yield (9:1 hexane/EtOAc) as a white solid: mp 118-119 ${ }^{\circ} \mathrm{C} ;{ }^{1} \mathrm{H}$ NMR $\left(\mathrm{CDCl}_{3}\right) \delta 3.02(\mathrm{t}, J=6.8 \mathrm{~Hz}, 4 \mathrm{H}), 4.00(\mathrm{t}, J=6.8 \mathrm{~Hz}, 4 \mathrm{H}), 5.23(\mathrm{~s}$, 2H), $5.41(\mathrm{~s}, 2 \mathrm{H}), 7.08(\mathrm{~s}, 2 \mathrm{H}), 7.26-7.29(\mathrm{~m}, 2 \mathrm{H}), 7.32-7.36(\mathrm{~m}, 4 \mathrm{H}), 7.44(\mathrm{~d}, J=7.6 \mathrm{~Hz}$, $4 \mathrm{H}) ;{ }^{13} \mathrm{C} \mathrm{NMR}\left(\mathrm{CDCl}_{3}\right) \delta 35.3,69.2,86.5,115.2,123.2,126.4,127.9,128.7,140.6$, 144.3, 152.9; IR $\left(\mathrm{CHCl}_{3}, \mathrm{~cm}^{-1}\right)$ 3089, 3019, 2933, 2914, 1622, 1571, 1484, 1448; HRMS Calcd for $\mathrm{C}_{26} \mathrm{H}_{24} \mathrm{IO}_{2}$ : 621.9866. Found: 621.9880 . 


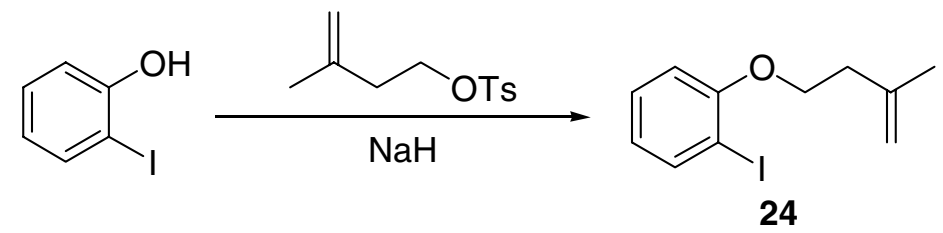

2-Iodophenyl 3-methyl-3-butenyl ether (24). Using the procedure used to prepare compound 1, but employing 2-iodophenol and 3-methyl-3-butenyl tosylate, afforded compound 24 in an $80 \%$ yield (30:1 hexane/EtOAc) as a colorless oil: ${ }^{1} \mathrm{H} \mathrm{NMR}\left(\mathrm{CDCl}_{3}\right)$ $\delta 1.84(\mathrm{~s}, 3 \mathrm{H}), 2.57(\mathrm{t}, J=6.9 \mathrm{~Hz}, 2 \mathrm{H}), 4.12(\mathrm{t}, J=6.9 \mathrm{~Hz}, 2 \mathrm{H}), 4.84-4.85(\mathrm{~m}, 1 \mathrm{H}), 4.85-$ $4.86(\mathrm{~m}, 1 \mathrm{H}), 6.70(\mathrm{dt}, J=1.5,7.5 \mathrm{~Hz}, 1 \mathrm{H}), 6.81(\mathrm{dd}, J=1.2,8.4 \mathrm{~Hz}, 1 \mathrm{H}), 7.25-7.31(\mathrm{~m}$, $1 \mathrm{H}), 7.76(\mathrm{dd}, J=1.5,7.8 \mathrm{~Hz}, 1 \mathrm{H}) ;{ }^{13} \mathrm{C} \mathrm{NMR}\left(\mathrm{CDCl}_{3}\right) \delta 23.2,37.3,68.2,86.9,112.3$, 112.6, 122.7, 129.6, 139.7, 142.3, 157.7; IR $\left(\mathrm{CHCl}_{3}, \mathrm{~cm}^{-1}\right) 3021,1610$; HRMS Calcd for $\mathrm{C}_{11} \mathrm{H}_{13} \mathrm{IO}: 273.9855$. Found: 273.9860 .

General procedure for the 1,4-palladium alkyl to aryl migration. A mixture of aryl halide $(0.25 \mathrm{mmol}), \mathrm{Pd}(\mathrm{OAc})_{2}(2.8 \mathrm{mg}, 0.0125 \mathrm{mmol})$, bis(diphenylphosphino)methane ( $4.8 \mathrm{mg}, 0.0125 \mathrm{mmol})$, cesium pivalate $(0.1170 \mathrm{~g}, 0.5$ $\mathrm{mmol}$ ), and the indicated amounts of alkene (if employed) in dry DMF (4 mL) was quickly flushed with argon and stirred at the indicated reaction temperature. The reaction was monitored by TLC and gas chromatographic analysis. After the reaction was complete, it was diluted with ethyl ether, washed with satd aq $\mathrm{NaHCO}_{3}$, dried over $\mathrm{Na}_{2} \mathrm{SO}_{4}$, concentrated, and purified by flash chromatography on silica gel.

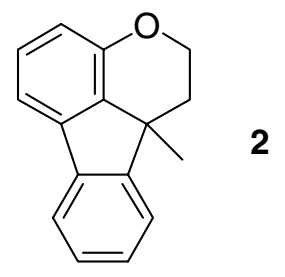


10b-Methyl-1,10b-dihydro-2H-indeno[1,2,3-de]chromene (2). The reaction mixture was chromatographed using 40:1 hexane/EtOAc to afford $49 \mathrm{mg}$ of the indicated

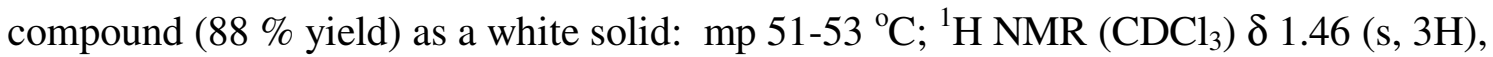
$1.63-1.70(\mathrm{ddd}, J=8.0,11.2,11.2 \mathrm{~Hz}, 1 \mathrm{H}), 2.29-2.33(\mathrm{ddd}, J=0.8,8.0,11.2 \mathrm{~Hz}, 1 \mathrm{H})$, $4.56(\mathrm{ddd}, J=0.8,8.0,13.2 \mathrm{~Hz}, 1 \mathrm{H}), 4.65(\mathrm{ddd}, J=8.0,11.2,13.2 \mathrm{~Hz}, 1 \mathrm{H}), 6.71-6.75$ (m, 1H), 7.22-7.26 (m, 2H), 7.29 (dd, $J=1.2,7.2 \mathrm{~Hz}, 1 \mathrm{H}), 7.33-7.37(\mathrm{~m}, 1 \mathrm{H}), 7.42-7.44(\mathrm{~m}$, 1H), 7.69-7.72 (m, 1H); ${ }^{13} \mathrm{C} \mathrm{NMR}\left(\mathrm{CDCl}_{3}\right) \delta 25.7,31.0,42.6,65.7,112.5,113.8,121.3$, 123.2, 127.1, 127.5, 129.5, 134.9, 140.4, 140.6, 153.0, 153.9; IR $\left(\mathrm{CHCl}_{3}, \mathrm{~cm}^{-1}\right) 3053$, 3010, 2963, 2919, 1614, 1592, 1486, 1447; HRMS Calcd for $\mathrm{C}_{16} \mathrm{H}_{14} \mathrm{O}$ : 222.1045. Found: 222.1048

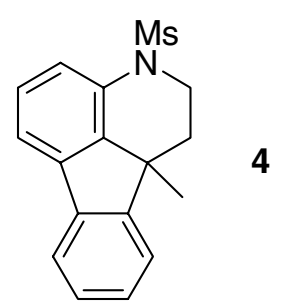

10b-Methyl-3-trifluoromethanesulfonyl-1,2,3,10b-tetrahydroindeno[1,2,3-

de]quinoline (4). The reaction mixture was chromatographed using 40:1 hexane/EtOAc to afford $71 \mathrm{mg}$ of the indicated compound (95\% yield) as a white solid: $\mathrm{mp} 111-112{ }^{\circ} \mathrm{C}$; ${ }^{1} \mathrm{H}$ NMR $\left(\mathrm{CDCl}_{3}\right) \delta 1.42(\mathrm{~s}, 3 \mathrm{H}), 1.45-1.58(\mathrm{~m}, 1 \mathrm{H}), 2.49-2.55(\mathrm{~m}, 1 \mathrm{H}), 3.94-4.11(\mathrm{~m}$, 2H), 7.29-7.40 (m, 3H), 7.43-7.45 (m, 2H), $7.57(\mathrm{~d}, J=8.1 \mathrm{~Hz}, 1 \mathrm{H}), 7.71-7.74(\mathrm{~m}, 1 \mathrm{H})$;

${ }^{13} \mathrm{C} \mathrm{NMR}\left(\mathrm{CDCl}_{3}\right) \delta 22.7,31.9,37.7,44.5,45.4,115.8,118.1,121.2,123.1,127.6,127.8$, $129.4,134.4,139.6,139.8,152.6$ (one $\mathrm{sp}^{2}$ carbon missing due to overlap); IR $\left(\mathrm{CHCl}_{3}\right.$, 
$\mathrm{cm}^{-1}$ ) 3023, 2967, 2927, 1591, 1451, 1346; HRMS Calcd for $\mathrm{C}_{17} \mathrm{H}_{17} \mathrm{NO}_{2} \mathrm{~S}: 299.0980$.

Found: 299.0987.

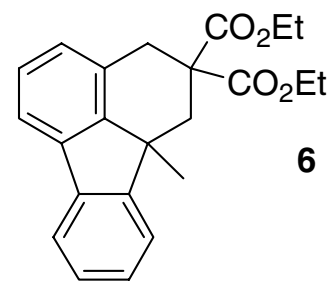

Dimethyl 10b-methyl-3,10b-dihydrofluoranthene-2,2(1H)-dicarboxylate (6). The reaction mixture was chromatographed using 15:1 hexane/EtOAc to afford $75 \mathrm{mg}$ of the indicated compound (82\% yield) as a colorless oil: ${ }^{1} \mathrm{H}$ NMR $\left(\mathrm{CDCl}_{3}\right) \delta 1.06(\mathrm{t}, J=6.9$ $\mathrm{Hz}, 3 \mathrm{H}), 1.33$ (t, $J=7.2 \mathrm{~Hz}, 3 \mathrm{H}), 1.89$ (d, $J=14.7 \mathrm{~Hz}, 1 \mathrm{H}), 3.09$ (d, $J=14.7 \mathrm{~Hz}, 1 \mathrm{H})$, $3.47(\mathrm{~d}, J=1.2 \mathrm{~Hz}, 2 \mathrm{H}), 3.90-4.00(\mathrm{~m}, 2 \mathrm{H}), 4.32(\mathrm{dq}, J=1.2,7.2 \mathrm{~Hz}, 2 \mathrm{H}), 7.15(\mathrm{~d}, J=$ 7.5 Hz, 1H), 7.25-7.37 (m, 3H), 7.48-7.51 (m, 1H), 7.56 (d, J = 7.5 Hz, 1H), 7.70-7.72 (m, $1 \mathrm{H}) ;{ }^{13} \mathrm{C} \mathrm{NMR}\left(\mathrm{CDCl}_{3}\right) \delta 14.0,14.3,24.3,33.3,38.6,46.2,56.6,61.7,62.0,118.8,121.0$, $123.4,126.0,127.3,127.5,128.0,132.6,138.2,140.2,149.4,154.4,172.0,172.9 ;$ IR (neat, $\mathrm{cm}^{-1}$ ) 2978, 2930, 1732, 1446; HRMS Calcd for $\mathrm{C}_{23} \mathrm{H}_{24} \mathrm{O}_{4}$ : 364.1674. Found: 364.1684 .

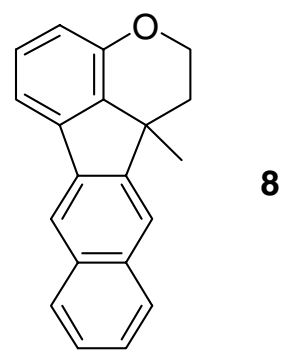

12b-Methyl-1,12b-dihydro-2H-benzo[5,6]indeno[1,2,3-de $]$ chromene (8). The reaction mixture was chromatographed using 30:1 hexane/EtOAc to afford $57 \mathrm{mg}$ of the 
indicated compound $\left(84 \%\right.$ yield) as a white solid: $\mathrm{mp} 154-156{ }^{\circ} \mathrm{C} ;{ }^{1} \mathrm{H} \mathrm{NMR}\left(\mathrm{CDCl}_{3}\right) \delta$ $1.52(\mathrm{~s}, 3 \mathrm{H}), 1.76-1.87(\mathrm{~m}, 1 \mathrm{H}), 2.41(\mathrm{dd}, J=3.6,12.9 \mathrm{~Hz}, 1 \mathrm{H}), 4.59(\mathrm{dd}, J=6.0,11.4$ $\mathrm{Hz}, 1 \mathrm{H}), 4.63-4.73(\mathrm{~m}, 1 \mathrm{H}), 6.76(\mathrm{~d}, J=11.1 \mathrm{~Hz}, 1 \mathrm{H}), 7.25-7.30(\mathrm{~m}, 1 \mathrm{H}), 7.37$ (d, $J=7.5$ $\mathrm{Hz}, 1 \mathrm{H}), 7.45-7.48(\mathrm{~m}, 2 \mathrm{H}), 7.81(\mathrm{~s}, 1 \mathrm{H}), 7.81-7.85(\mathrm{~m}, 1 \mathrm{H}), 7.88-7.91(\mathrm{~m}, 1 \mathrm{H}), 8.11(\mathrm{~s}$, $1 \mathrm{H}) ;{ }^{13} \mathrm{C} \mathrm{NMR}\left(\mathrm{CDCl}_{3}\right) \delta 26.9,31.5,42.1,65.6,113.1,114.3,119.5,121.6,125.9,125.9$, $128.1,128.5,129.7,133.1,133.6,135.4,139.1,140.0,151.9,153.0 ; \mathrm{IR}\left(\mathrm{CHCl}_{3}, \mathrm{~cm}^{-1}\right)$ 3017, 2965, 1614, 1591, 1484; HRMS Calcd for $\mathrm{C}_{20} \mathrm{H}_{16} \mathrm{O}: 272.1201$. Found: 272.1208 .

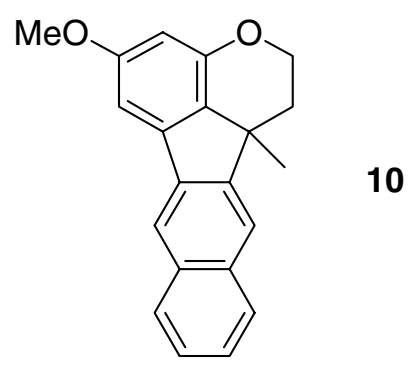

\section{5-Methoxy-12b-methyl-1,12b-dihydro-2H-benzo[5,6]indeno[1,2,3-de]chromene}

(10). The reaction mixture was chromatographed using 10:1 hexane/EtOAc to afford 69 mg of the indicated compound (91\% yield) as a white solid: $\mathrm{mp} 58-61{ }^{\circ} \mathrm{C} ;{ }^{1} \mathrm{H}$ NMR $\left(\mathrm{CDCl}_{3}\right) \delta 1.50(\mathrm{~s}, 3 \mathrm{H}), 1.71-1.83(\mathrm{~m}, 1 \mathrm{H}), 2.34-2.39(\mathrm{~m}, 1 \mathrm{H}), 4.53-4.58(\mathrm{~m}, 1 \mathrm{H}), 4.61-$ $4.70(\mathrm{~m}, 1 \mathrm{H}), 6.36(\mathrm{~d}, J=2.1 \mathrm{H}, 1 \mathrm{H}), 6.95(\mathrm{~d}, J=2.1 \mathrm{~Hz}, 1 \mathrm{H}), 7.44-7.47(\mathrm{~m}, 2 \mathrm{H}), 7.78(\mathrm{~s}$, 1H), 7.81-7.84 (m, 1H), 7.87-7.91 (m, 1H), $8.07(\mathrm{~s}, 1 \mathrm{H}) ;{ }^{13} \mathrm{C} \mathrm{NMR}\left(\mathrm{CDCl}_{3}\right) \delta$ 27.1, 31.9, 41.6, 56.0, 65.8, 99.1, 100.4, 119.5, 121.6, 125.9, 126.0, 128.2, 128.5, 133.2, 133.6, 139.1, 140.4, 152.4, 153.5, 162.2 (one sp ${ }^{2}$ carbon missing due to overlap); IR $\left(\mathrm{CHCl}_{3}, \mathrm{~cm}^{-1}\right)$ 3018, 2963, 2922, 1612, 1596, 1483; HRMS Calcd for $\mathrm{C}_{21} \mathrm{H}_{18} \mathrm{O}_{2}$ : 302.1307. Found: 302.1312 . 


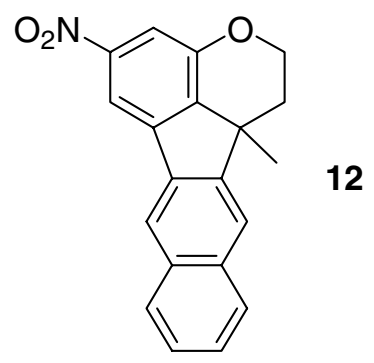

12b-Methyl-5-nitro-1,12b-dihydro-2H-benzo[5,6]indeno[1,2,3-de]chromene (12).

The reaction mixture was chromatographed using 15:1 hexane/EtOAc to afford $18 \mathrm{mg}$ of the indicated compound (21\% yield) as a yellow solid: mp 192-194 ${ }^{\circ} \mathrm{C} ;{ }^{1} \mathrm{H}$ NMR $\left(\mathrm{CDCl}_{3}\right) \delta 1.55(\mathrm{~s}, 3 \mathrm{H}), 1.79-1.90(\mathrm{~m}, 1 \mathrm{H}), 2.50(\mathrm{dd}, J=3.3,12.9 \mathrm{~Hz}, 1 \mathrm{H}), 4.65-4.79(\mathrm{~m}$, 2H), 7.50-7.55 (m, 1H), $7.65(\mathrm{~d}, J=1.5 \mathrm{~Hz}, 1 \mathrm{H}), 7.87-7.89(\mathrm{~m}, 2 \mathrm{H}), 7.94-7.97(\mathrm{~m}, 1 \mathrm{H})$, 8.22-8.23 (m, 2H); ${ }^{13} \mathrm{C} \mathrm{NMR}\left(\mathrm{CDCl}_{3}\right) \delta 26.7,31.0,42.5,66.3,108.5,110.2,120.8,122.1$, $126.5,126.8,128.2,128.8,133.5,133.6,137.0,140.5,141.6,150.3,150.9,152.8 ;$ IR $\left(\mathrm{CHCl}_{3}, \mathrm{~cm}^{-1}\right) 3019,2971,1617,1525$; HRMS Calcd for $\mathrm{C}_{20} \mathrm{H}_{15} \mathrm{O}_{3}: 317.1052$. Found: 317.1058.

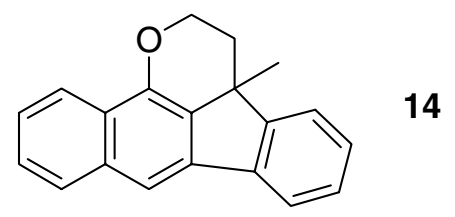

3a-Methyl-3,3a-dihydro-2H-benzo $[h]$ indeno[1,2,3-de $]$ chromene (14). The reaction mixture was chromatographed using 19:1 hexane/EtOAc to afford $65 \mathrm{mg}$ of the indicated compound (95\% yield) as a yellow oil: ${ }^{1} \mathrm{H}$ NMR $\left(\mathrm{CDCl}_{3}\right) \delta 1.50(\mathrm{~s}, 3 \mathrm{H}), 1.73-$ $1.84(\mathrm{~m}, 1 \mathrm{H}), 2.36-2.42(\mathrm{~m}, 1 \mathrm{H}), 4.72-4.87(\mathrm{~m}, 2 \mathrm{H}), 7.29-7.48(\mathrm{~m}, 5 \mathrm{H}), 7.67(\mathrm{~s}, 1 \mathrm{H})$, 7.81-7.88 (m, 2H), 8.10-8.13 (m, 1H); ${ }^{13} \mathrm{C} \mathrm{NMR}\left(\mathrm{CDCl}_{3}\right) \delta$ 27.0, 31.2, 42.2, 66.1, 110.8, $121.3,121.8,123.5,124.5,124.9,126.0,127.7,127.8,128.0,128.6,135.8,138.8,140.0$, 
147.6, 154.2; IR $\left(\mathrm{CDCl}_{3}, \mathrm{~cm}^{-1}\right)$ 3053, 2963, 2920, 1646, 1587, 1509; HRMS Calcd for $\mathrm{C}_{20} \mathrm{H}_{14} \mathrm{O}: 272.1201$. Found: 272.1207 .

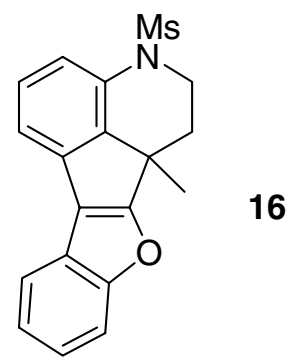

\section{6a-Methyl-4-trifluoromethanesulfonyl-4,5,6,6a-}

tetrahydro[1]benzofuro[3',2':4,5]cyclopenta[1,2,3-de]quinoline (16). The reaction mixture was chromatographed using 4:1 hexane/EtOAc to afford $65 \mathrm{mg}$ of the indicated compound (76\% yield) as a yellow oil: ${ }^{1} \mathrm{H} \mathrm{NMR}\left(\mathrm{CDCl}_{3}\right) \delta 1.55-1.62(\mathrm{~m}, 4 \mathrm{H}), 2.58(\mathrm{dd}$, $J=1.2 \mathrm{~Hz}, 5.7 \mathrm{~Hz}, 1 \mathrm{H}), 3.95-4.11(\mathrm{~m}, 2 \mathrm{H}), 7.31-7.38(\mathrm{~m}, 4 \mathrm{H}), 7.51-7.58(\mathrm{~m}, 2 \mathrm{H}), 7.77-$ $7.81(\mathrm{~m}, 1 \mathrm{H}) ;{ }^{13} \mathrm{C} \mathrm{NMR}\left(\mathrm{CDCl}_{3}\right) \delta 19.3,30.4,38.0,41.3,44.9,112.7,115.7,116.9,120.3$, 122.3, 123.6, 123.8, 124.2, 129.5, 134.2, 135.2, 138.2, 160.5, 172.2; IR $\left(\mathrm{CHCl}_{3}, \mathrm{~cm}^{-1}\right)$ 3020, 2927, 1722, 1610, 1592, 1487; HRMS Calcd for $\mathrm{C}_{19} \mathrm{H}_{17} \mathrm{O}_{3} \mathrm{SN}$ : 339.0929. Found: 339.0935 .

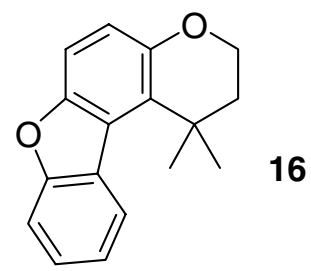

1,1-Dimethyl-2,3-dihydro-1 $H$-[1] benzofuro[3,2-f]chromene (16). The reaction mixture was chromatographed using 30:1 hexane/EtOAc to afford $52 \mathrm{mg}$ of the indicated compound $\left(83 \%\right.$ yield) as a colorless oil: ${ }^{1} \mathrm{H}$ NMR $\left(\mathrm{CDCl}_{3}\right) \delta 1.69(\mathrm{~s}, 6 \mathrm{H}), 1.98-2.02(\mathrm{~m}$, 
2H), 4.14-4.18 (m, 2H), $7.99(\mathrm{~d}, J=9.0 \mathrm{~Hz}, 1 \mathrm{H}), 7.34(\mathrm{~d}, J=9.0 \mathrm{~Hz}, 1 \mathrm{H}), 7.31-7.36(\mathrm{~m}$, 1H), 7.40-7.46 (m, 1H), 7.55-7.58 (m, 1H), $8.21(\mathrm{dd}, J=0.9,8.4 \mathrm{~Hz}, 1 \mathrm{H}) ;{ }^{13} \mathrm{C} \mathrm{NMR}$ $\left(\mathrm{CDCl}_{3}\right) \delta 28.4,31.7,41.6,62.9,110.7,112.0,118.2,122.0,122.0,124.1,125.8,126.3$, 126.4, 150.5, 151.8, 157.0; IR (neat, $\mathrm{cm}^{-1}$ ) 3069, 2959, 1622, 1585, 1472, 1415; HRMS Calcd for $\mathrm{C}_{17} \mathrm{H}_{16} \mathrm{O}_{2}: 252.1150$. Found: 252.1156 .

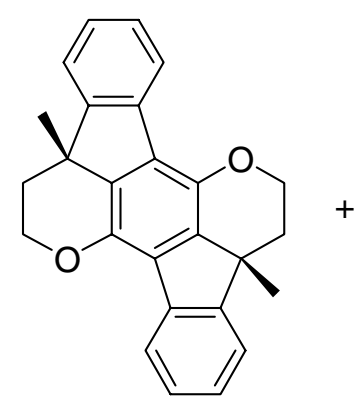

21

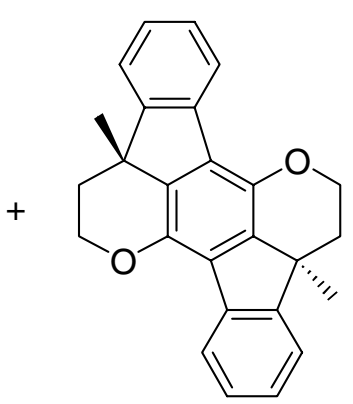

22

3a,10a-Dimethyl-2,3,3a,9,10,10a-hexahydro-1,8-dioxarubicenes (21 and 22). The reaction mixture was chromatographed using 30:1 hexane/EtOAc to afford $52 \mathrm{mg}$ of the indicated compounds as a 60:40 mixture (48\% yield) as a yellow solid. Compound 21 was confirmed by enantiomeric separation on HPLC using Cyclobond I 2000 RSP (250 x $4.6 \mathrm{~mm})$. Compound 21: ${ }^{1} \mathrm{H}$ NMR $\left(\mathrm{CDCl}_{3}\right) \delta 1.53(\mathrm{~s}, 6 \mathrm{H}), 1.64(\mathrm{ddd}, J=6.0,13.2,13.2$ $\mathrm{Hz}, 2 \mathrm{H}), 2.30(\mathrm{dd}, J=3.0,13.2 \mathrm{~Hz}, 2 \mathrm{H}), 4.62(\mathrm{dd}, J=6.0,10.4 \mathrm{~Hz}, 2 \mathrm{H}), 4.69-4.79(\mathrm{~m}$, 2H), 7.20-7.25 (m, 2H), 7.31-7.36 (m, 2H), $7.40(\mathrm{~d}, J=7.5 \mathrm{~Hz}, 2 \mathrm{H}), 7.96(\mathrm{~d}, J=7.5 \mathrm{~Hz}$, $2 \mathrm{H}) ;{ }^{13} \mathrm{C} \mathrm{NMR}\left(\mathrm{CDCl}_{3}\right) \delta 26.2,31.6,43.5,65.3,122.9,123.9,126.2,126.9,127.9,136.9$, 139.5, 143.9, 153.9; IR $\left(\mathrm{CDCl}_{3}, \mathrm{~cm}^{-1}\right)$ 2956, 1505, 1437; HRMS Calcd for $\mathrm{C}_{26} \mathrm{H}_{22} \mathrm{O}_{2}$ : 366.1620. Found: 366.1626. Compound 22: ${ }^{1} \mathrm{H}$ NMR $\left(\mathrm{CDCl}_{3}\right) \delta 1.46(\mathrm{~s}, 6 \mathrm{H}), 1.83-$ $1.94(\mathrm{~m}, 2 \mathrm{H}), 2.39(\mathrm{ddd}, J=1.2,3.6,13.2 \mathrm{~Hz}, 2 \mathrm{H}), 4.59-4.79(\mathrm{~m}, 4 \mathrm{H}), 7.18-7.26(\mathrm{~m}, 2 \mathrm{H})$, 
7.31-7.36 (m, 2H), $7.40(\mathrm{~d}, J=7.5 \mathrm{~Hz}, 2 \mathrm{H}), 7.93(\mathrm{~d}, J=7.5 \mathrm{~Hz}, 2 \mathrm{H})$; HRMS Calcd for $\mathrm{C}_{26} \mathrm{H}_{22} \mathrm{O}_{2}$ : 366.1620. Found: 366.1626 .

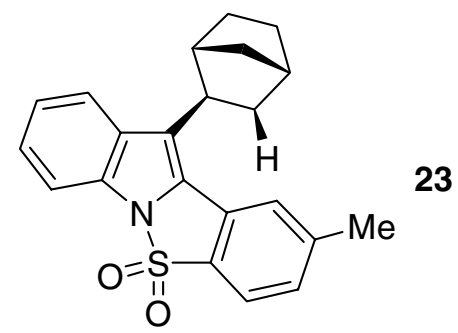

\section{8-Methyl-1H-indole[1,2-b]benzo[d]isothiazole-10-(endo-norbornan-2-yl)-5,5-}

dioxide (23). Compound $\mathbf{2 2}^{4}$ (99.3 mg, $0.25 \mathrm{mmol}$ ) and norbornene (25.9 mg, 0.275 mmol) were allowed to react under our standard reaction conditions for $1 \mathrm{~d}$. The reaction mixture was diluted with diethyl ether $(30 \mathrm{~mL})$ and washed with brine $(30 \mathrm{~mL})$. The organic layer was dried $\left(\mathrm{Na}_{2} \mathrm{SO}_{4}\right)$, filtered, and the solvent removed under reduced pressure to afford $68.0 \mathrm{~g}$ (75\% yield) of the indicated compound $\mathbf{2 3}$ as a yellow solid, which is easily recrystallized from $\mathrm{CHCl}_{3} /$ hexanes: $\mathrm{mp} 255-256{ }^{\circ} \mathrm{C} ;{ }^{1} \mathrm{H} \mathrm{NMR}\left(\mathrm{CDCl}_{3}\right) \delta$ 1.42-1.49 (m, 3H), 1.71-1.75 (m, 2H), 1.89-1.94 (m, 2H), 2.07-2.08 (m, 1H), 2.51 (br s, 4H), 2.65 (br s, 1H), 3.28-3.31 (m, 1H), 7.18-7.23 (m, 1H), 7.26-7.28 (m, 1H), 7.31-7.36 (m, 1H), $7.60(\mathrm{~s}, 1 \mathrm{H}), 7.68-7.72(\mathrm{~m}, 2 \mathrm{H}), 7.83(\mathrm{~d}, J=8.4 \mathrm{~Hz}, 1 \mathrm{H}) ;{ }^{13} \mathrm{C} \mathrm{NMR}\left(\mathrm{CDCl}_{3}\right) \delta$ $22.3,29.4,30.7,36.5,37.8,37.9,41.1,42.7,112.0,122.6,122.8,123.0,123.2,123.5$, $125.5,128.7,129.2,132.2,133.0,135.5,145.0$ (one $\mathrm{sp}^{2}$ carbon missing due to overlap); IR (neat, $\mathrm{cm}^{-1}$ ) 2952, 2866, 1597, 1326, 1179; HRMS Calcd for $\mathrm{C}_{22} \mathrm{H}_{21} \mathrm{NO}_{2} \mathrm{~S}: 363.1293$. Found: 363.1300 . 


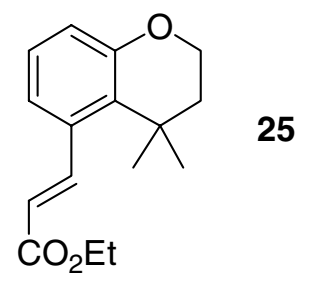

Ethyl (2E)-3-(4,4-dimethyl-3,4-dihydro-2H-chromen-5-yl)propenoate (25).

Compound $24(68.5 \mathrm{mg}, 0.25 \mathrm{mmol})$ and ethyl acrylate $(0.375 \mathrm{mmol})$ were allowed to react under our standard reaction conditions for $18 \mathrm{~h}$. The reaction mixture was diluted with diethyl ether $(30 \mathrm{~mL})$ and washed with brine $(30 \mathrm{~mL})$. The organic layer was dried $\left(\mathrm{Na}_{2} \mathrm{SO}_{4}\right)$, filtered and the solvent removed under reduced pressure. The residue was chromatographed (8:1 hexane/EtOAc) to afford $40.3 \mathrm{mg}$ of the indicated compound (62 $\%$ yield) as a yellow oil: ${ }^{1} \mathrm{H}$ NMR $\left(\mathrm{CDCl}_{3}\right) \delta 1.34(\mathrm{t}, J=7.2 \mathrm{~Hz}, 3 \mathrm{H}), 1.46(\mathrm{~s}, 6 \mathrm{H}), 1.84(\mathrm{t}, J$ $=7.2 \mathrm{~Hz}, 2 \mathrm{H}), 4.15(\mathrm{t}, J=7.2 \mathrm{~Hz}, 1 \mathrm{H}), 4.27(\mathrm{q}, J=7.2 \mathrm{~Hz}, 2 \mathrm{H}), 6.18(\mathrm{~d}, J=15.6 \mathrm{~Hz}, 1 \mathrm{H}), 6.84$ $(\mathrm{dd}, J=1.5,7.5 \mathrm{~Hz}, 1 \mathrm{H}), 6.98-7.10(\mathrm{~m}, 2 \mathrm{H}), 8.32(\mathrm{~d}, J=15.6 \mathrm{~Hz}, 1 \mathrm{H}) ;{ }^{13} \mathrm{C} \mathrm{NMR}\left(\mathrm{CDCl}_{3}\right) \delta$ $14.5,30.4,31.6,41.1,60.7,62.5,119.5,119.6,121.6,127.4,129.9,136.0,146.3,154.7,169.2$ IR $\left(\mathrm{CHCl}_{3}, \mathrm{~cm}^{-1}\right)$ 3018, 2966, 1712; HRMS Calcd for $\mathrm{C}_{16} \mathrm{H}_{20} \mathrm{O}_{3}:$ 260.1412. Found: 260.1416 .

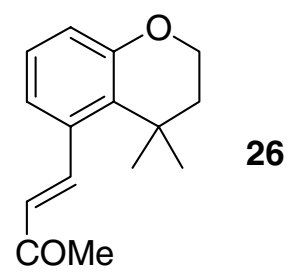

(3E)-4-(4,4-Dimethyl-3,4-dihydro-2H-chromen-5-yl)but-3-en-2-one (26). Compound 24 (68.5 mg, $0.25 \mathrm{mmol})$ and methyl vinyl ketone $(0.375 \mathrm{mmol})$ were allowed to react under our standard reaction conditions for $18 \mathrm{~h}$. The reaction mixture was diluted with diethyl ether $(30 \mathrm{~mL})$ and washed with brine $(30 \mathrm{~mL})$. The organic 
layer was dried $\left(\mathrm{Na}_{2} \mathrm{SO}_{4}\right)$, filtered and the solvent removed under reduced pressure. The residue was chromatographed (3:1 hexane/EtOAc) to afford $42.0 \mathrm{mg}$ of the indicated compound (73\% yield) as a yellow oil: ${ }^{1} \mathrm{H} \operatorname{NMR}\left(\mathrm{CDCl}_{3}\right) \delta 1.47(\mathrm{~s}, 6 \mathrm{H}), 1.85(\mathrm{t}, J=5.2 \mathrm{~Hz}$, 2H), $2.38(\mathrm{~s}, 3 \mathrm{H}), 4.15(\mathrm{t}, J=5.2 \mathrm{~Hz}, 2 \mathrm{H}), 6.47(\mathrm{~d}, J=15.0 \mathrm{~Hz}, 1 \mathrm{H}), 6.86(\mathrm{dd}, J=0.9,6.0 \mathrm{~Hz}$, 1H), $7.01(\mathrm{dd}, J=1.2,5.7 \mathrm{~Hz}, 1 \mathrm{H}), 7.09(\mathrm{t}, J=5.7 \mathrm{~Hz}, 1 \mathrm{H}), 8.17(\mathrm{~d}, J=15.0 \mathrm{~Hz}, 1 \mathrm{H}) ;{ }^{13} \mathrm{C} \mathrm{NMR}$ $\left(\mathrm{CDCl}_{3}\right) \delta 28.2,30.5,31.6,41.1,62.5,119.8,121.6,127.5,128.3,130.1,135.9,144.8,154.7$,

198.3; IR $\left(\mathrm{CHCl}_{3}, \mathrm{~cm}^{-1}\right) 3020,2964,1705$; HRMS Calcd for $\mathrm{C}_{15} \mathrm{H}_{18} \mathrm{O}_{2}$ : 230.1307. Found: 230.1311.

\section{References}

(1) Tsukayama, M.; Utsumi, H.; Kunugi, A.; Nozaki, H. Heterocycles 1997, 45, 1131.

(2) Zhu, G.-D.; Staeger, M. A.; Boyd, S. A. Org. Lett. 2000, 2, 3345.

(3) Ramos, A. M.; Rispens, M. T.; van Duren, J. K. J.; Hummelen, J. C.; Janssen, R. A. J. J. Am. Chem. Soc. 2001, 123, 6714.

(4) (a) Campo, M. A.; Larock, R. C. Org. Lett. 2000, 2, 3675. (b) Campo, M. A.; Larock, R. C. J. Org. Chem. 2002, 67, 5616.

(5) Padwa, A.; Brodney, M. A.; Dimitroff, M.; Liu, B.; Wu, T. J. Org. Chem. 2001, $66,3119$. 

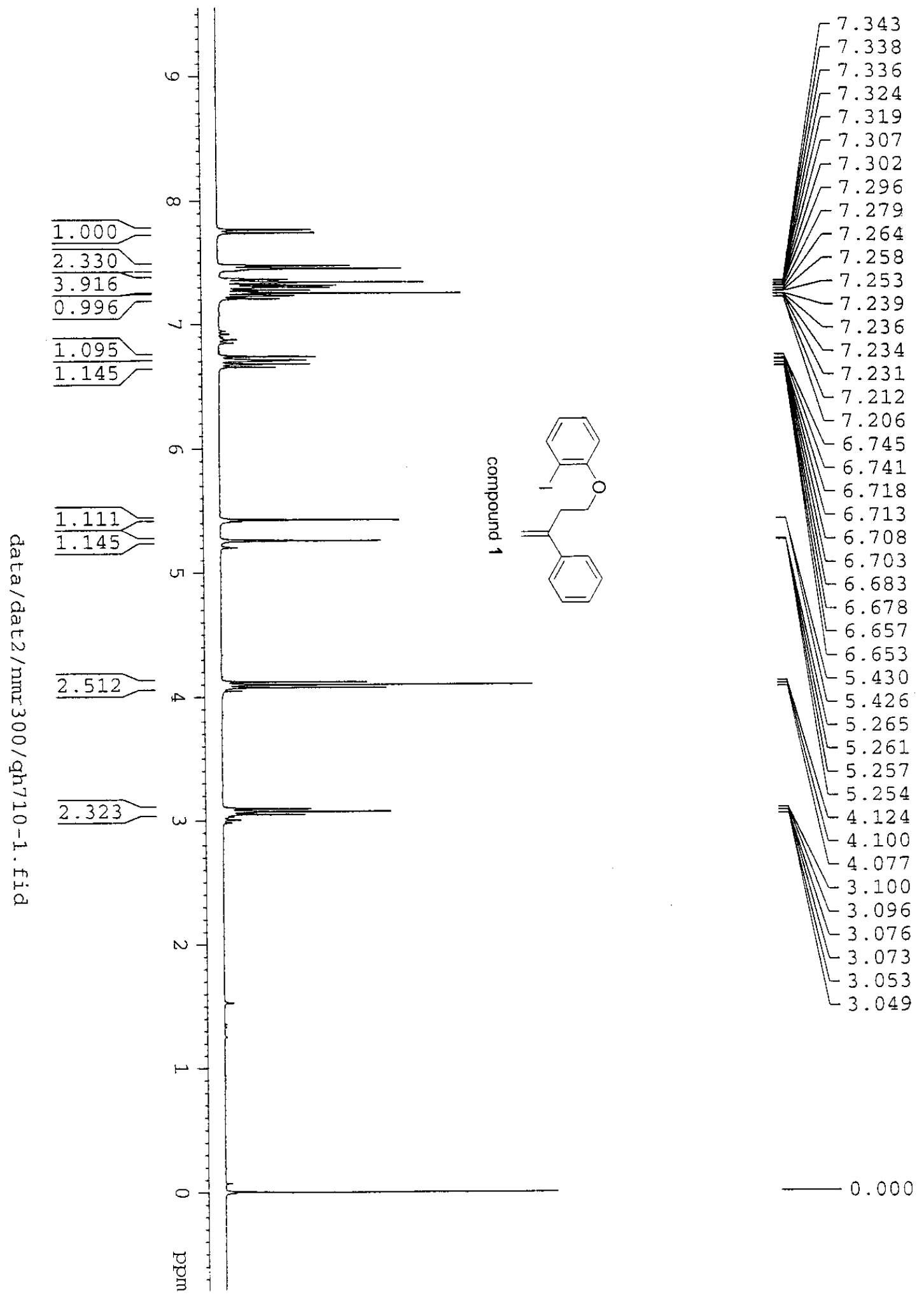


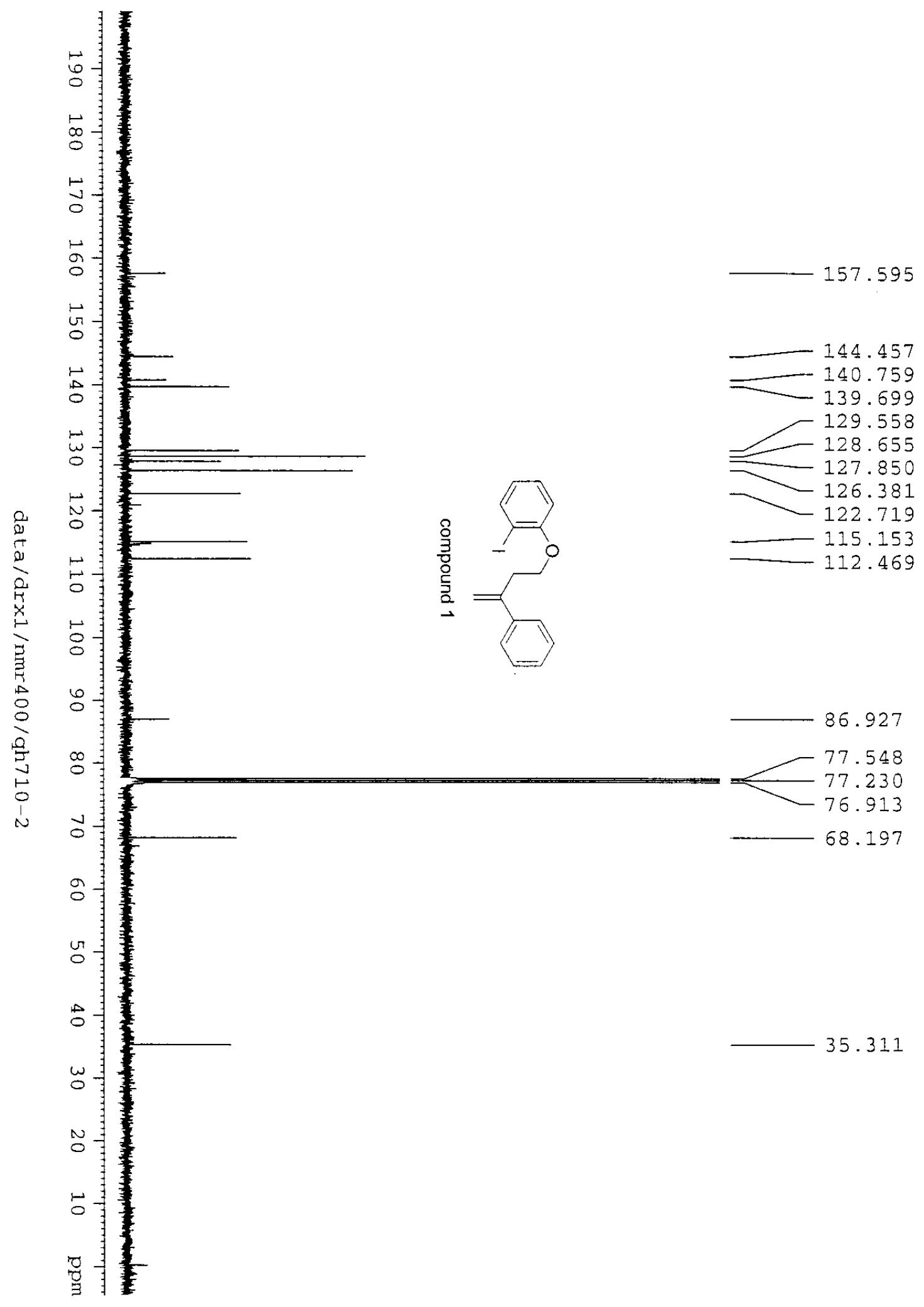




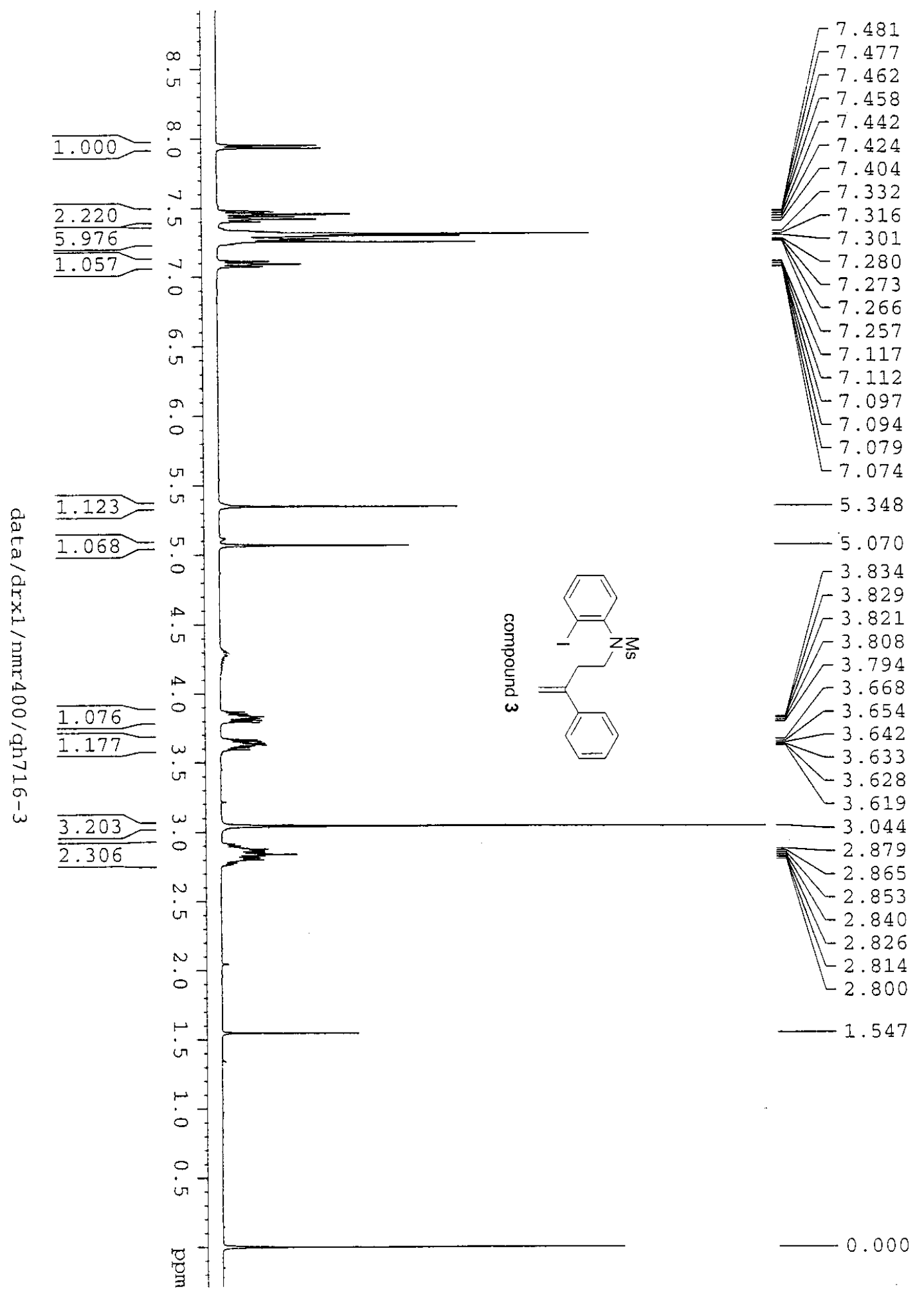




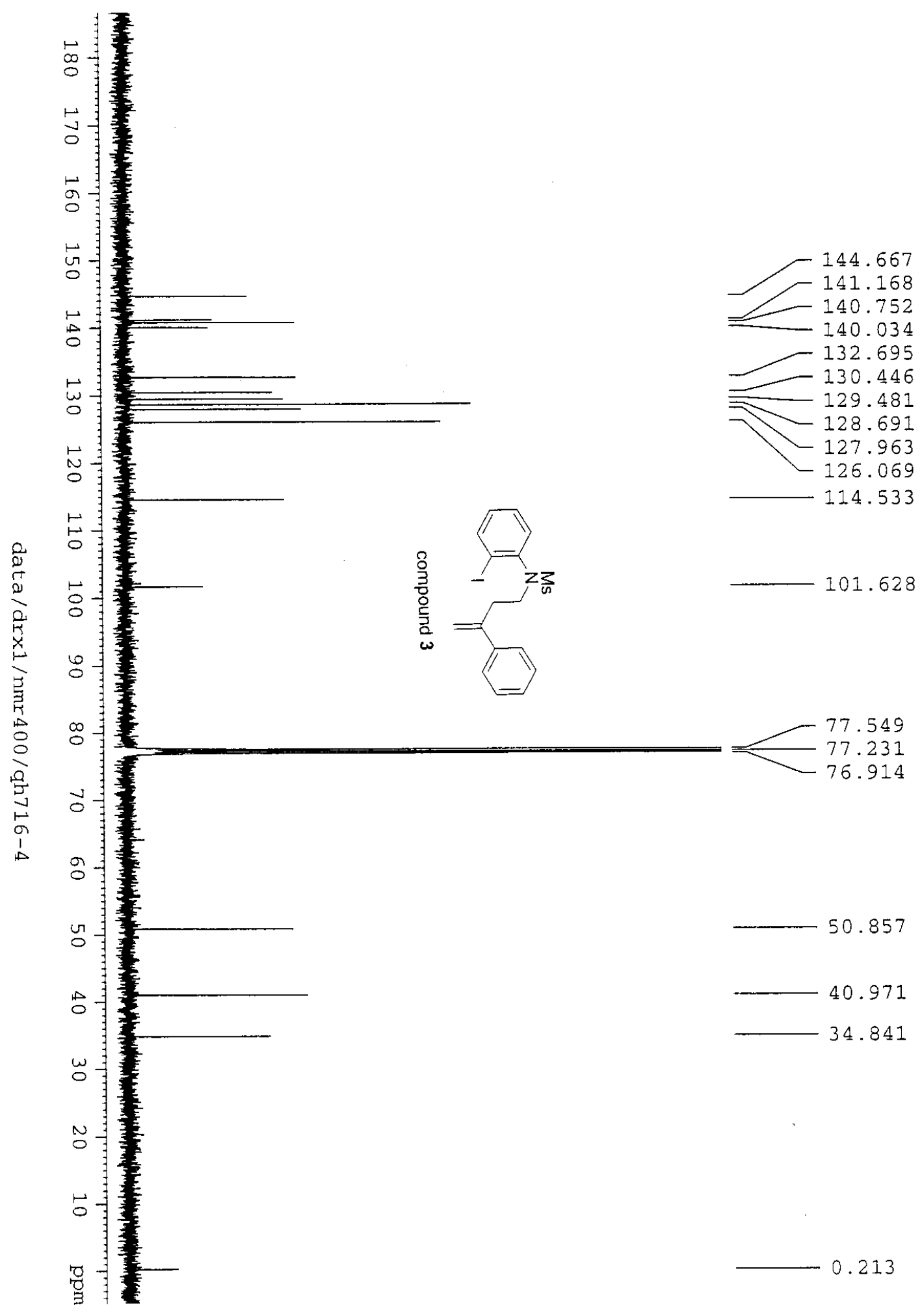




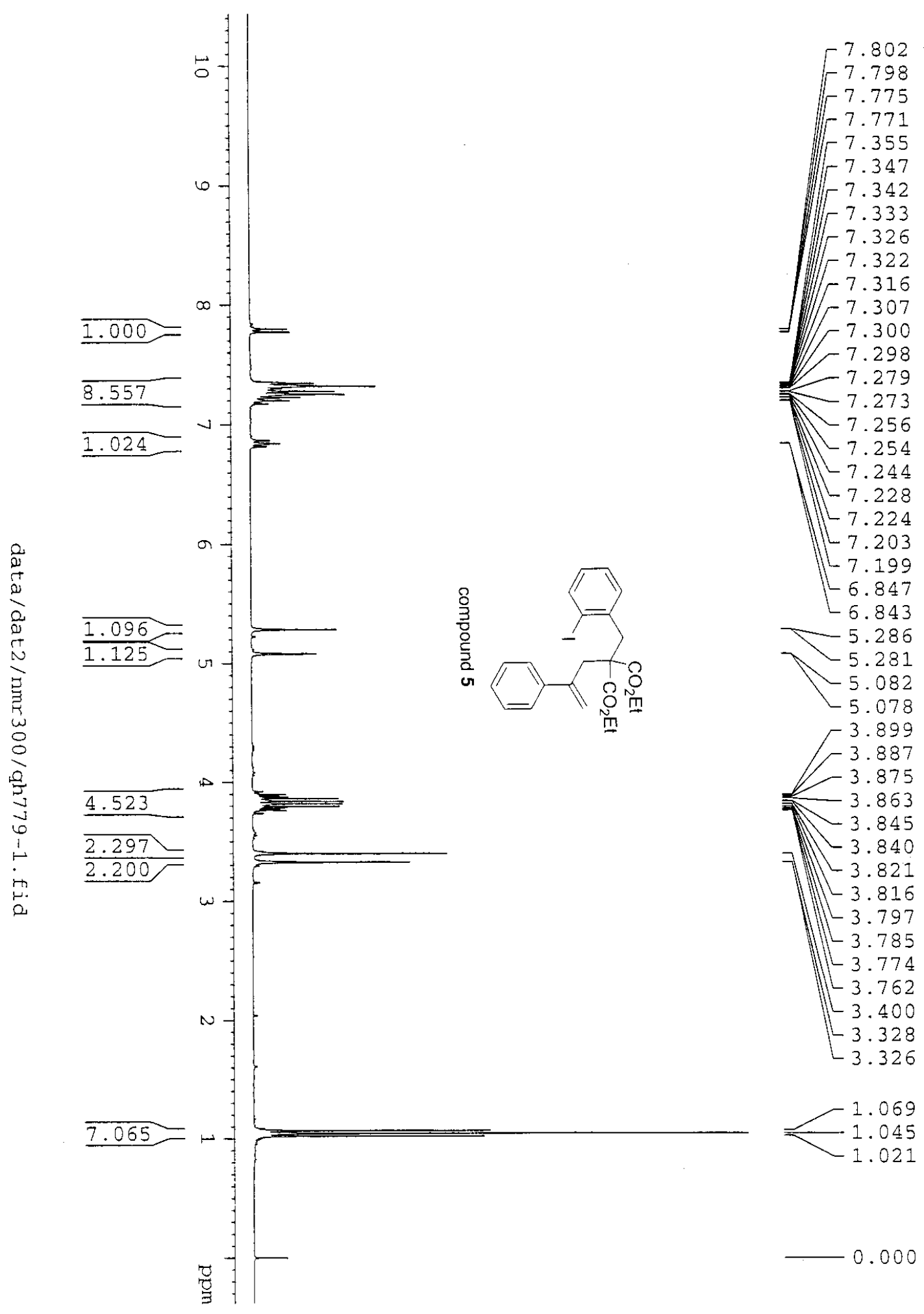




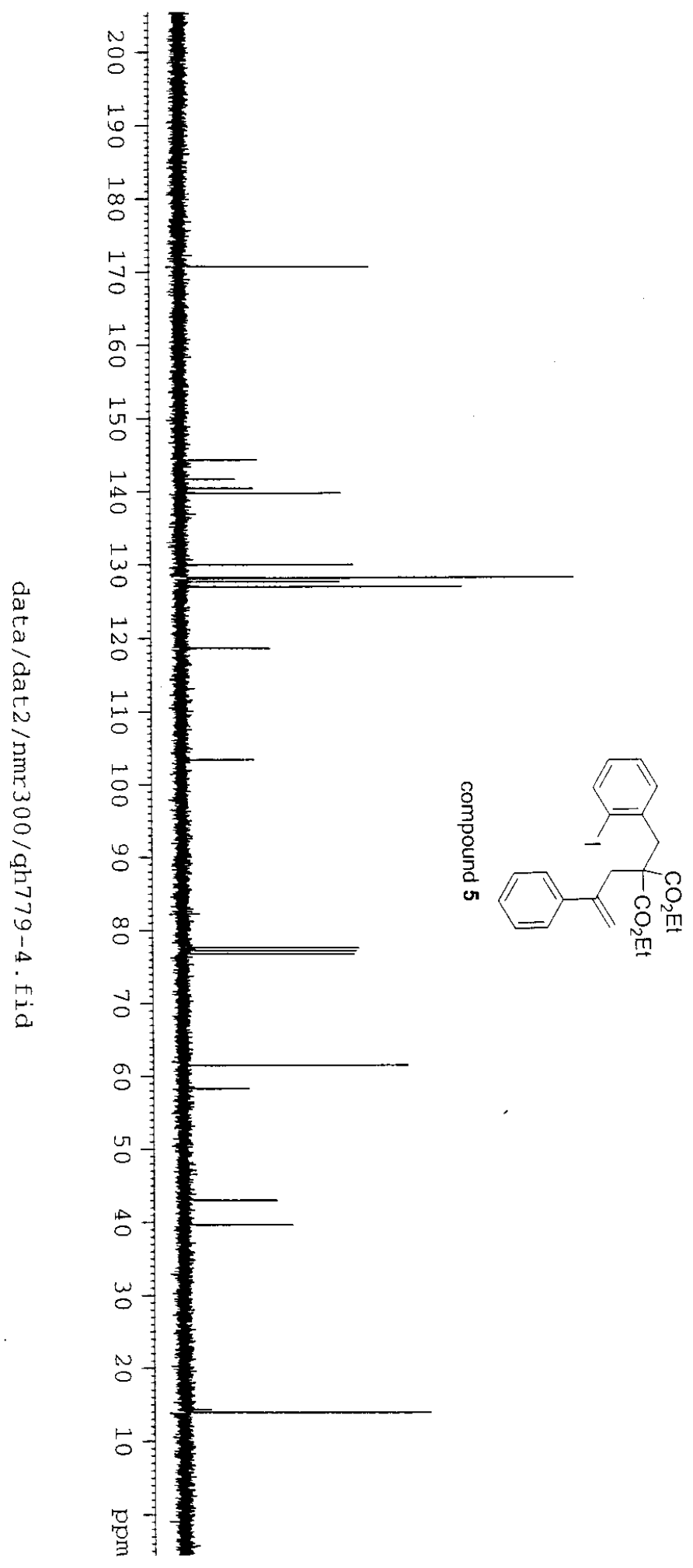

170.732
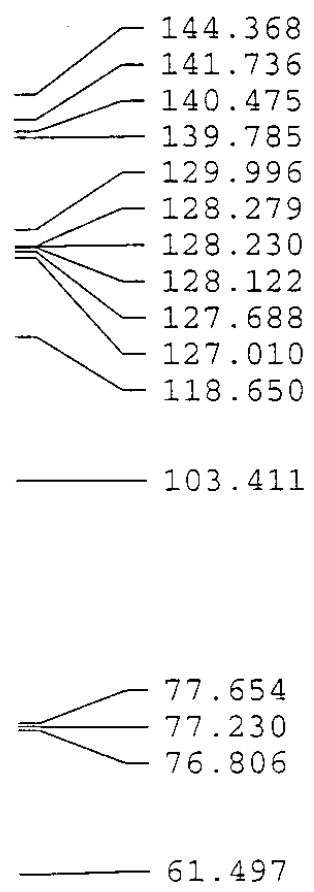

58.265

43.009

$\square$
$\longrightarrow$ 9.645

13.863 

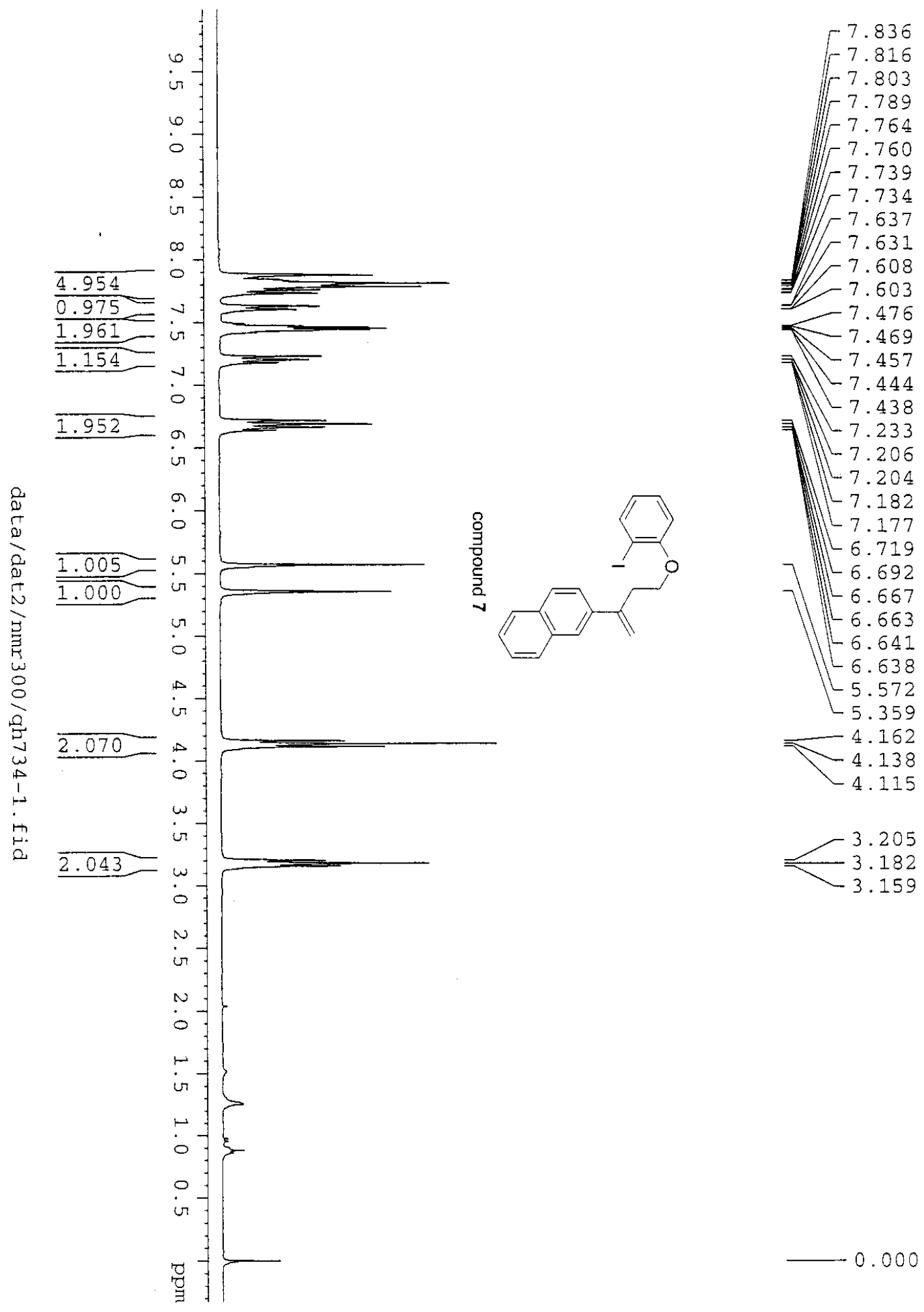

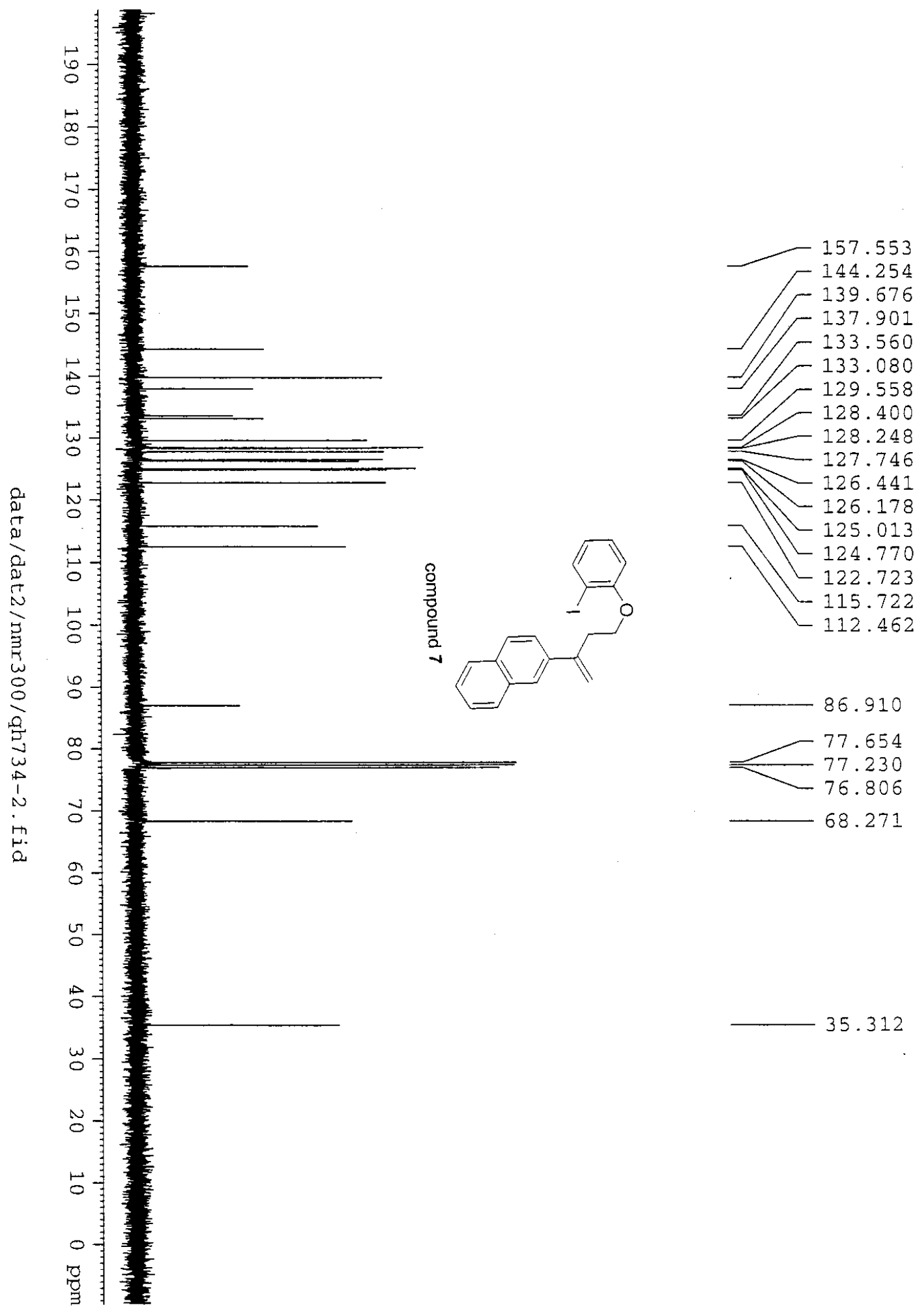


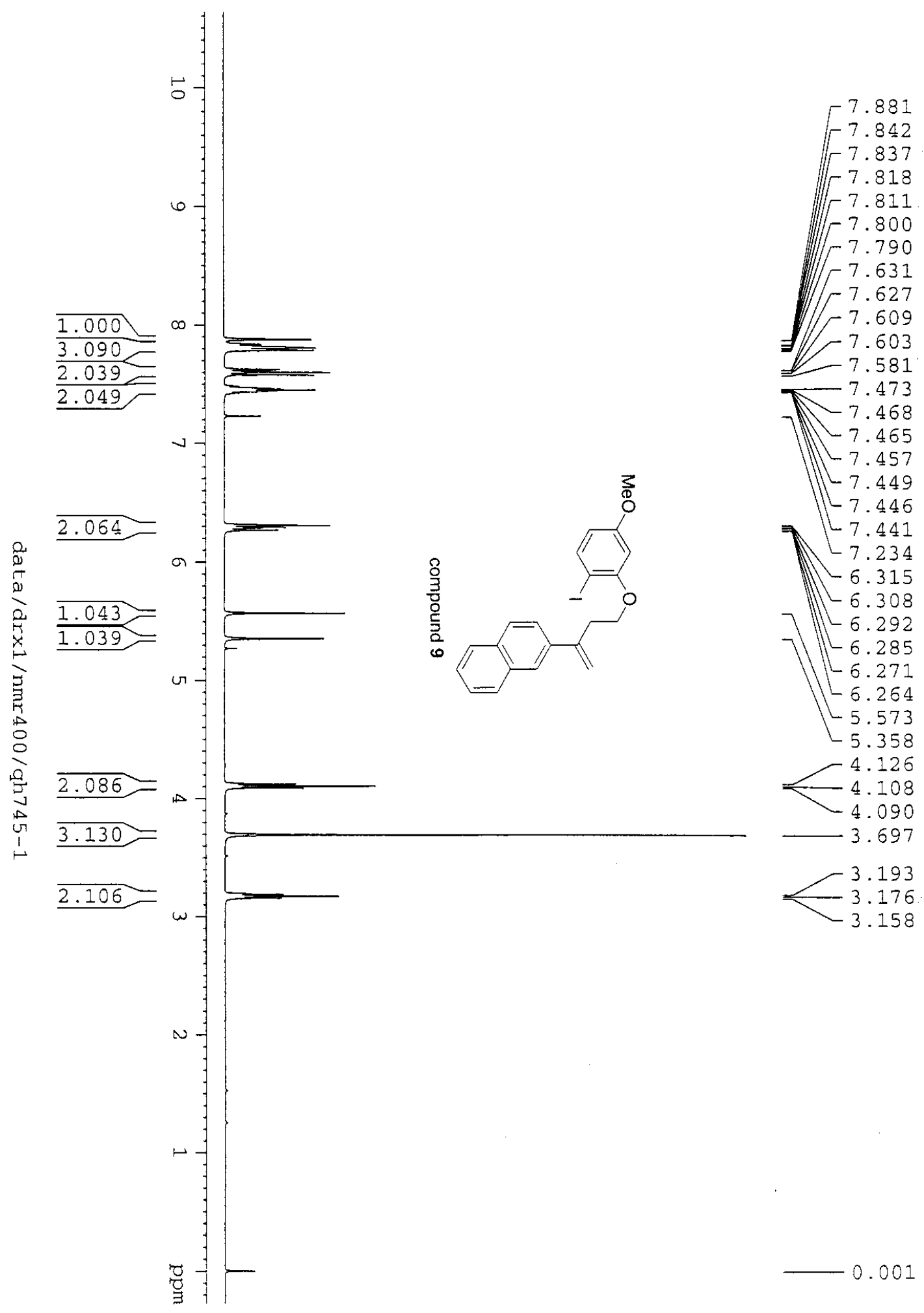




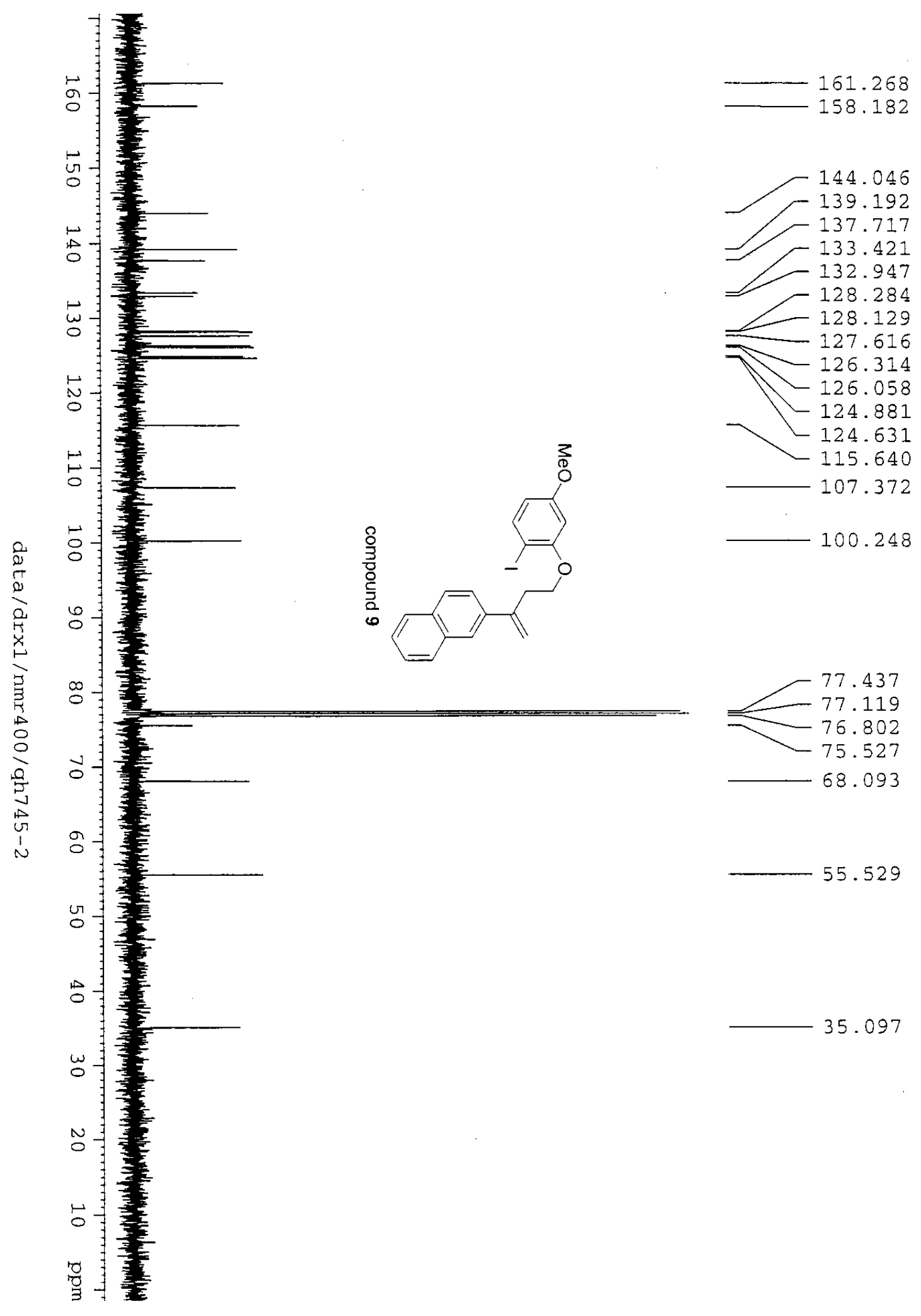



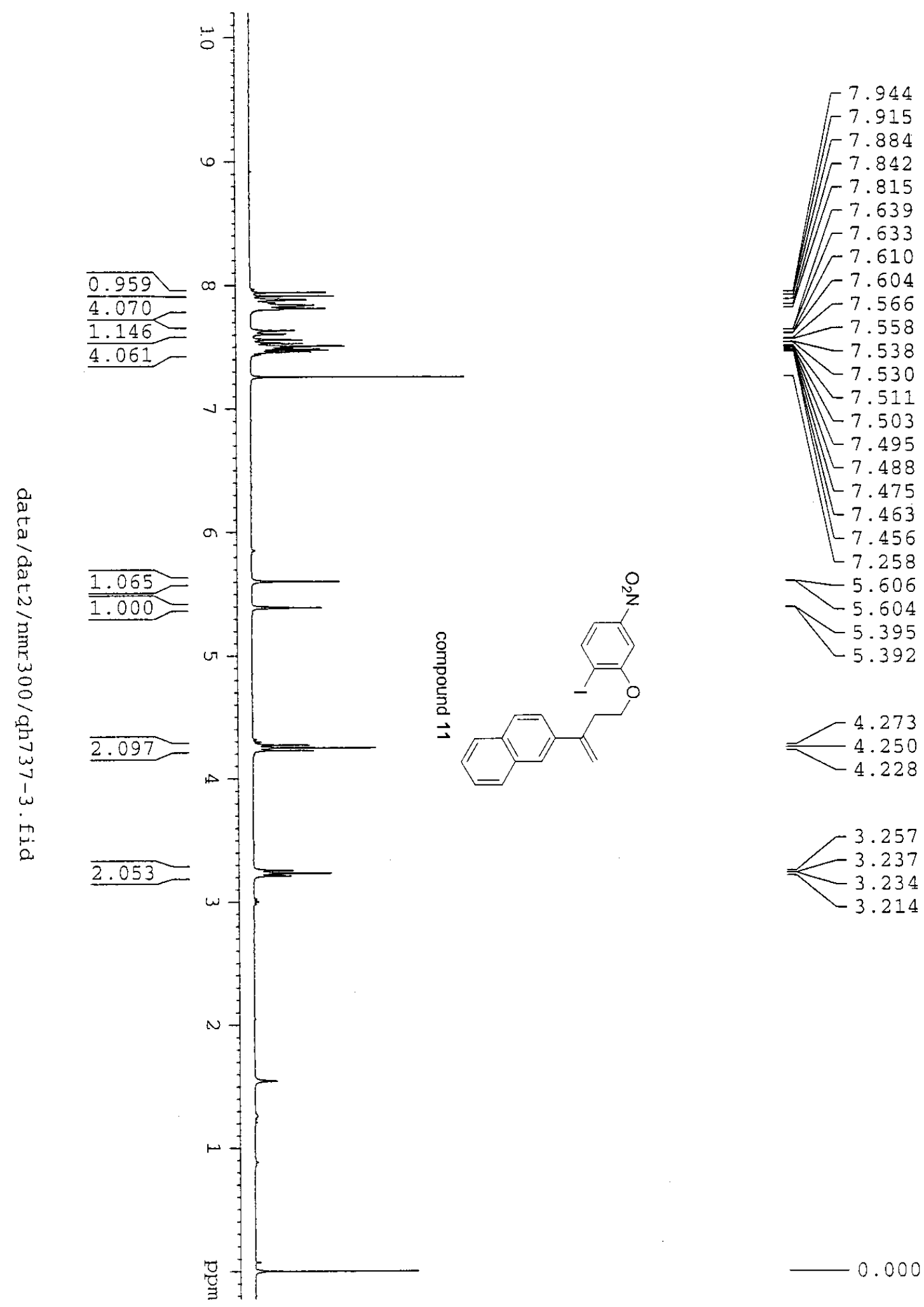


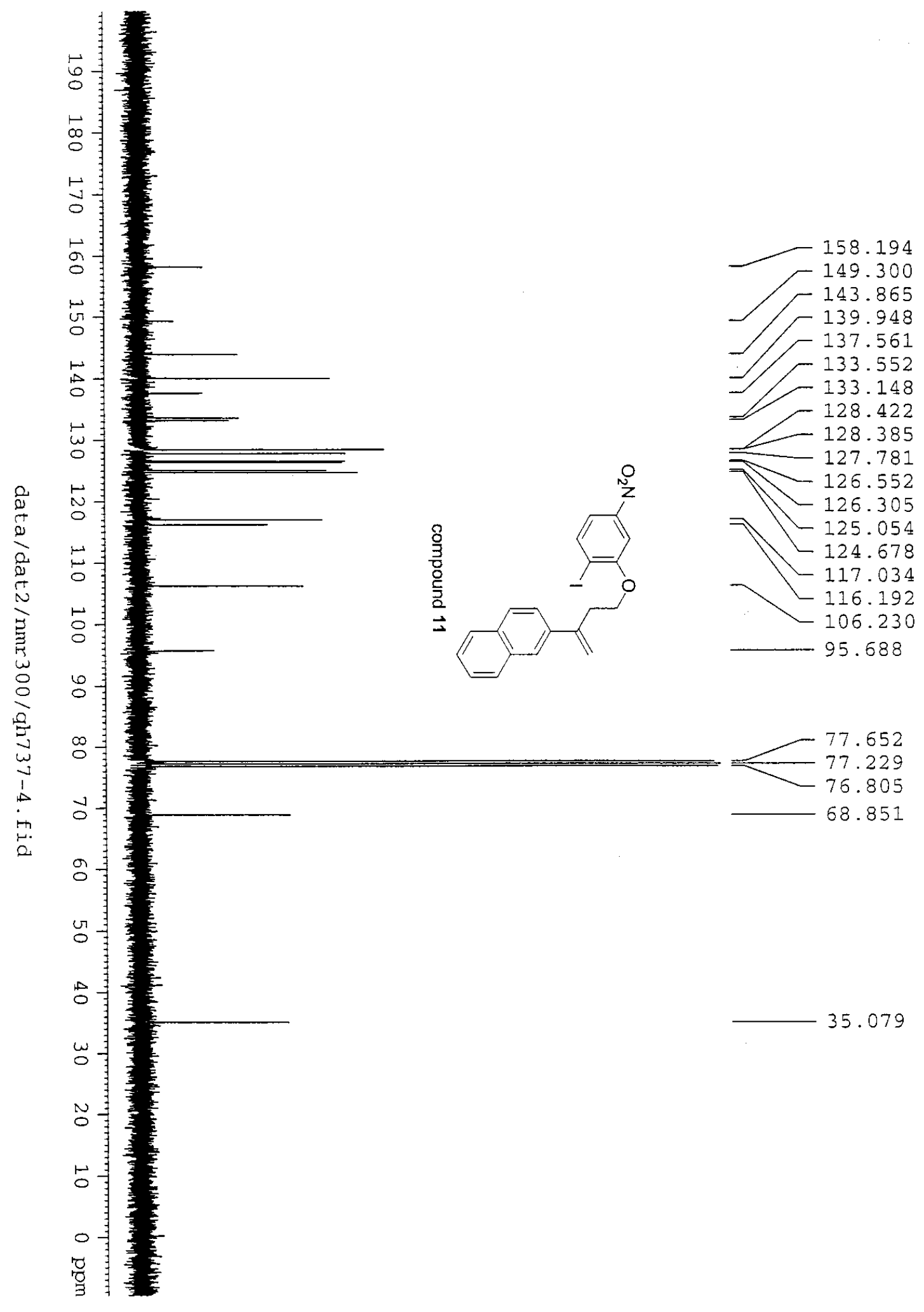



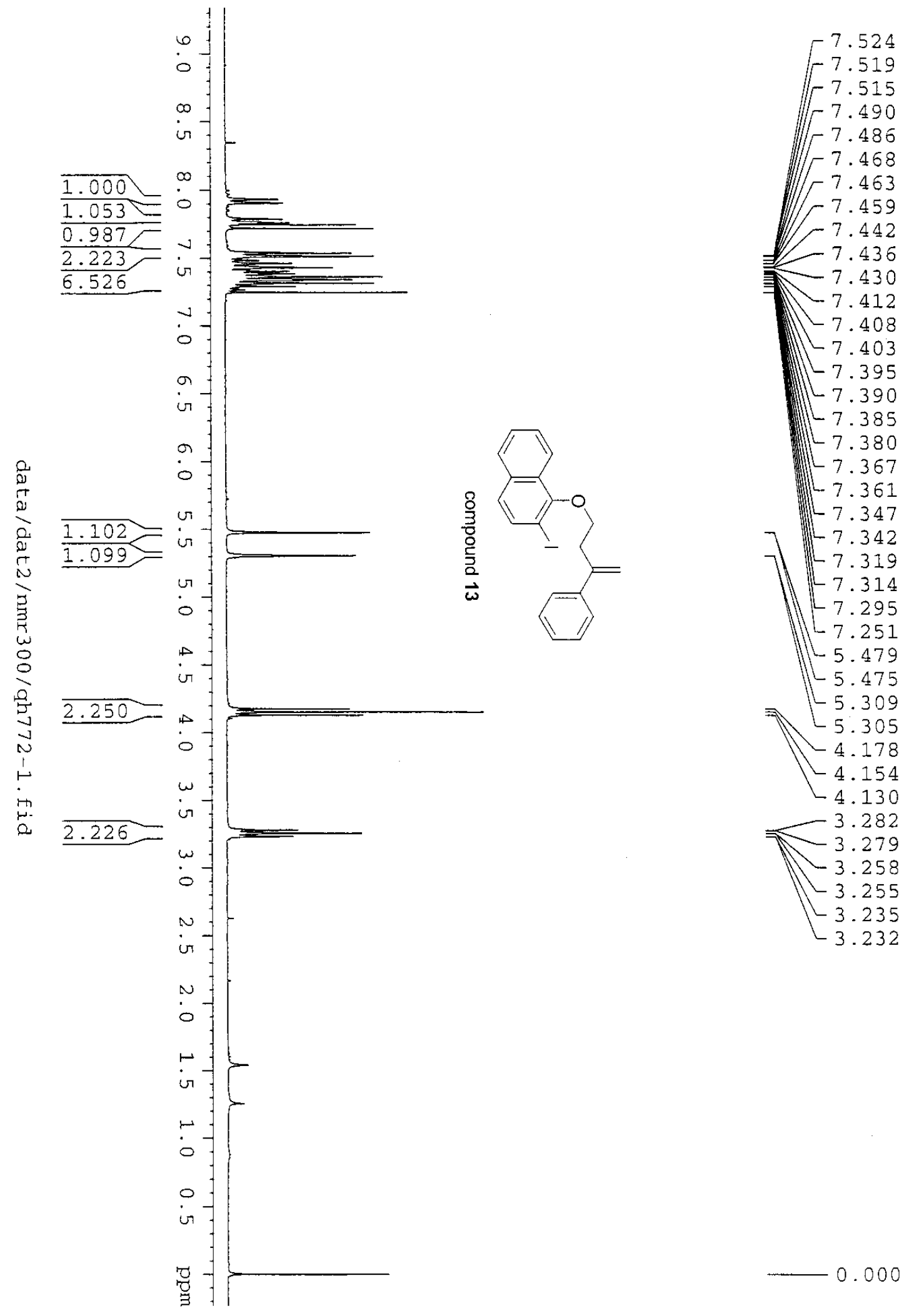


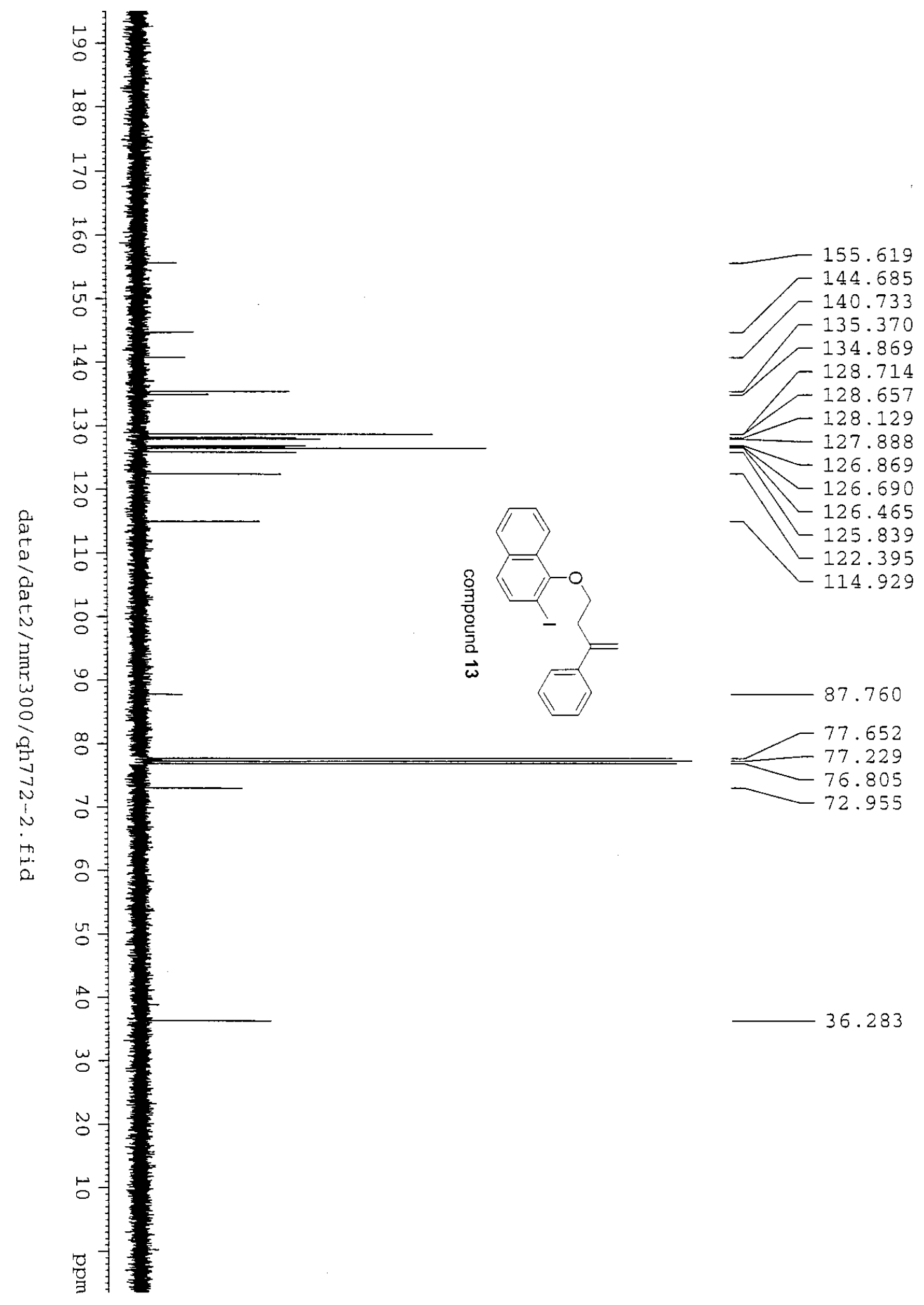




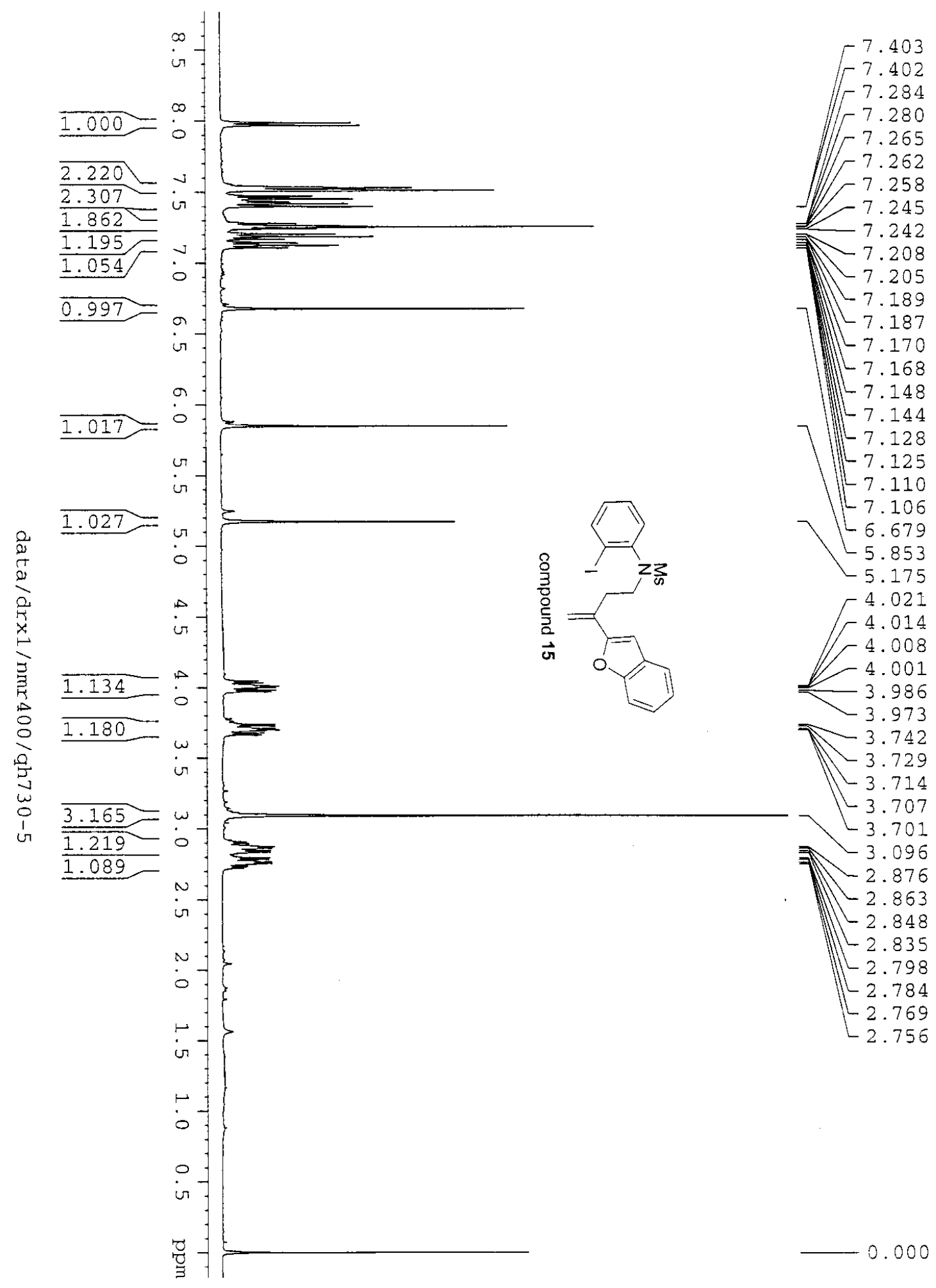




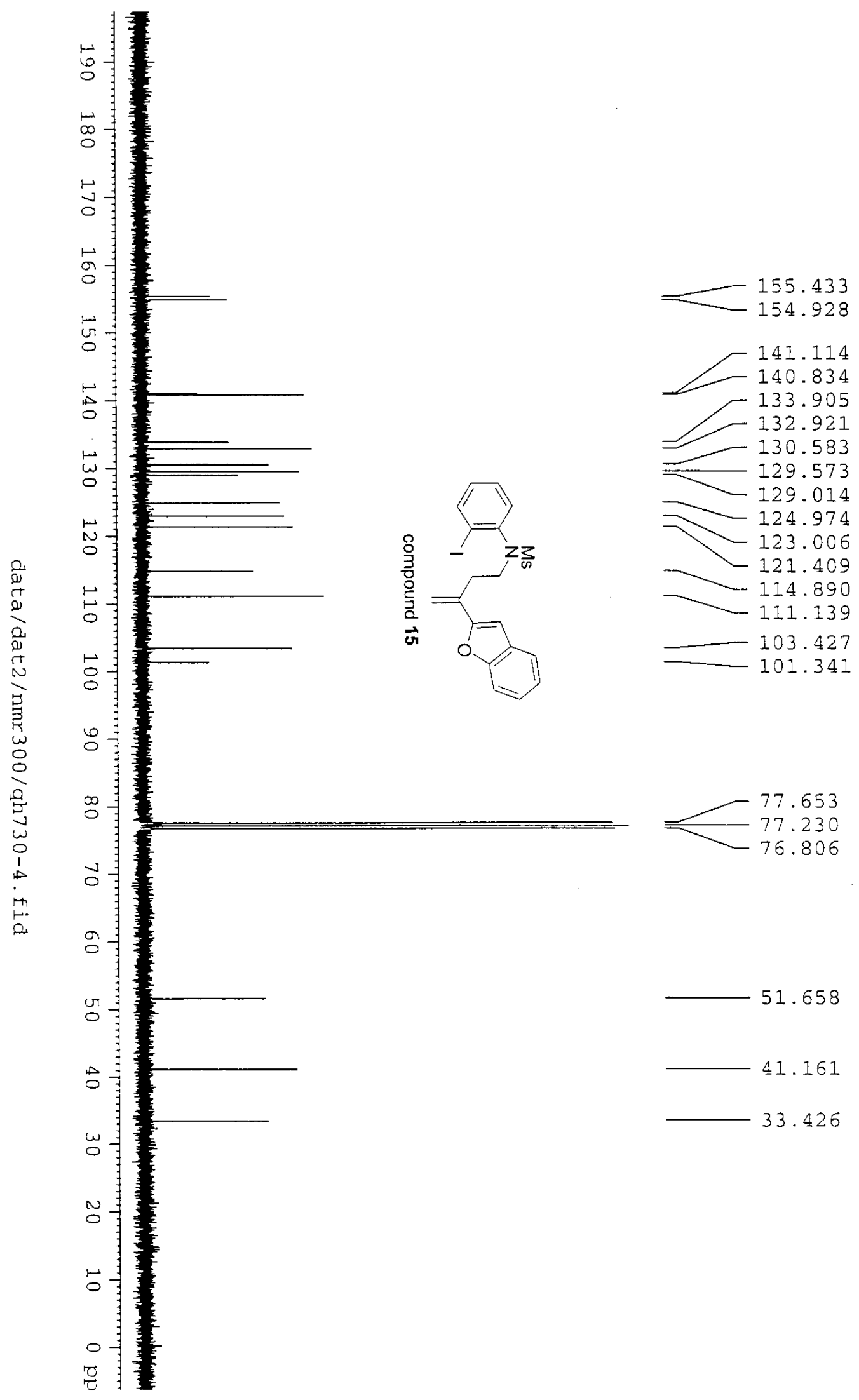



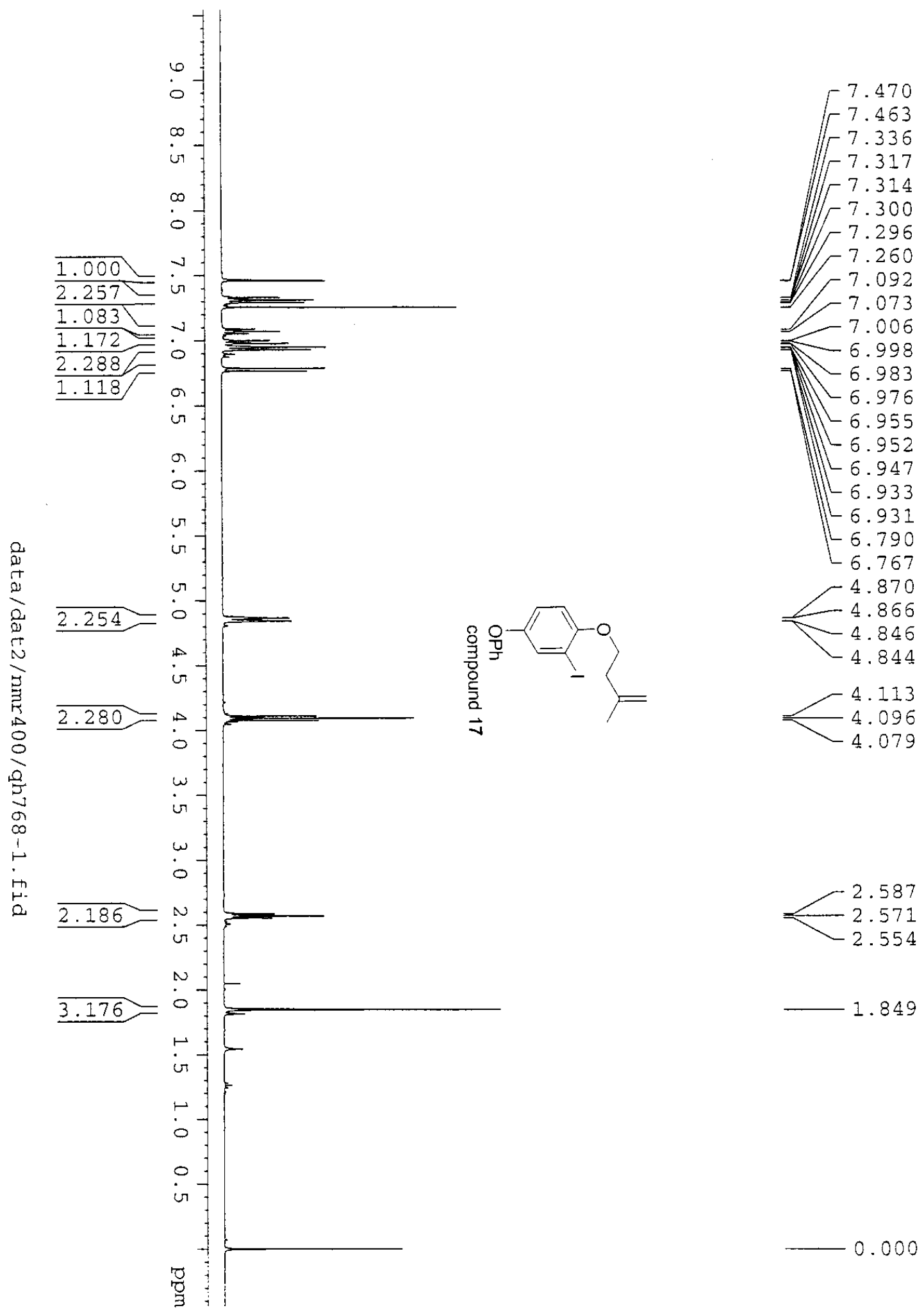


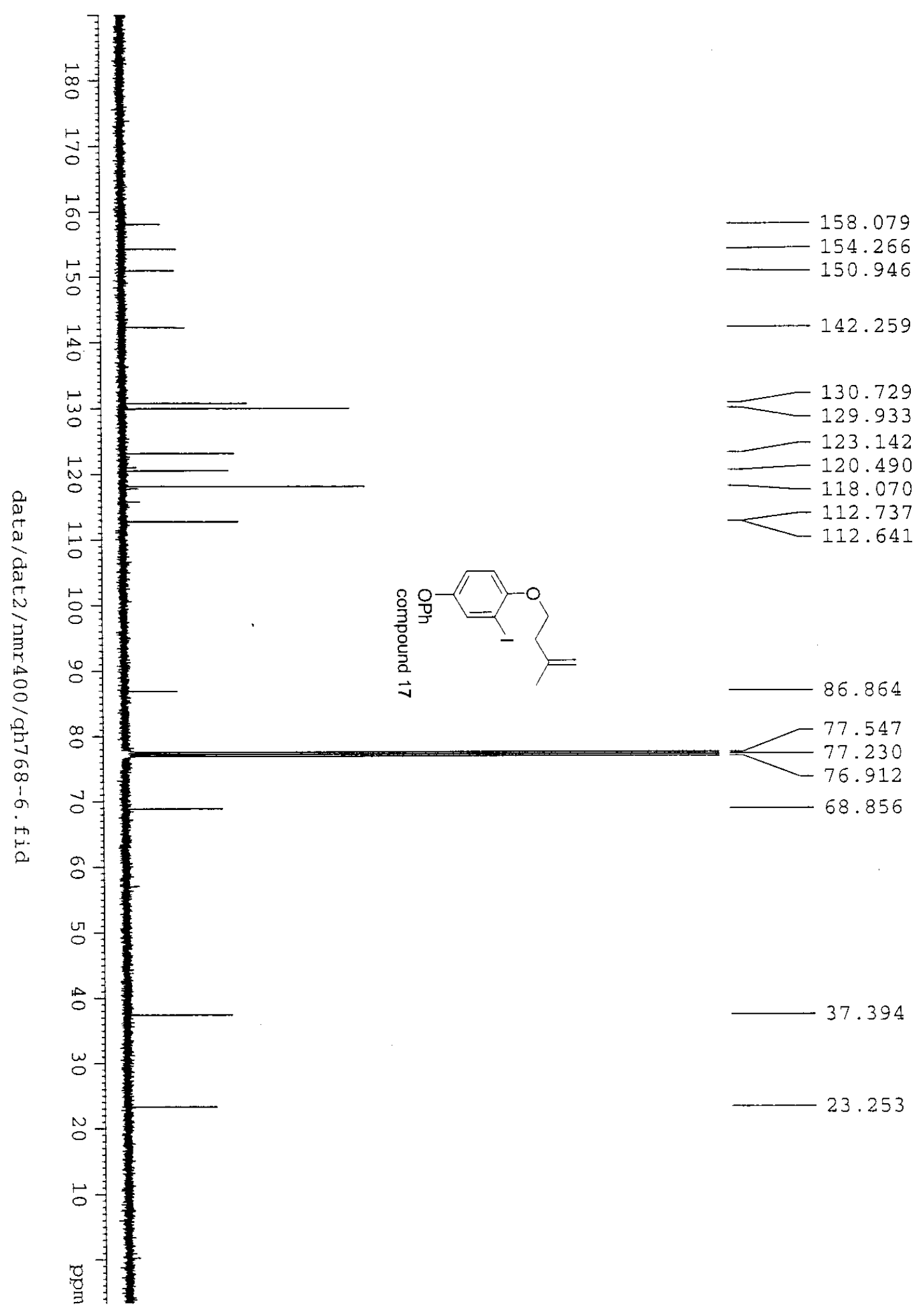



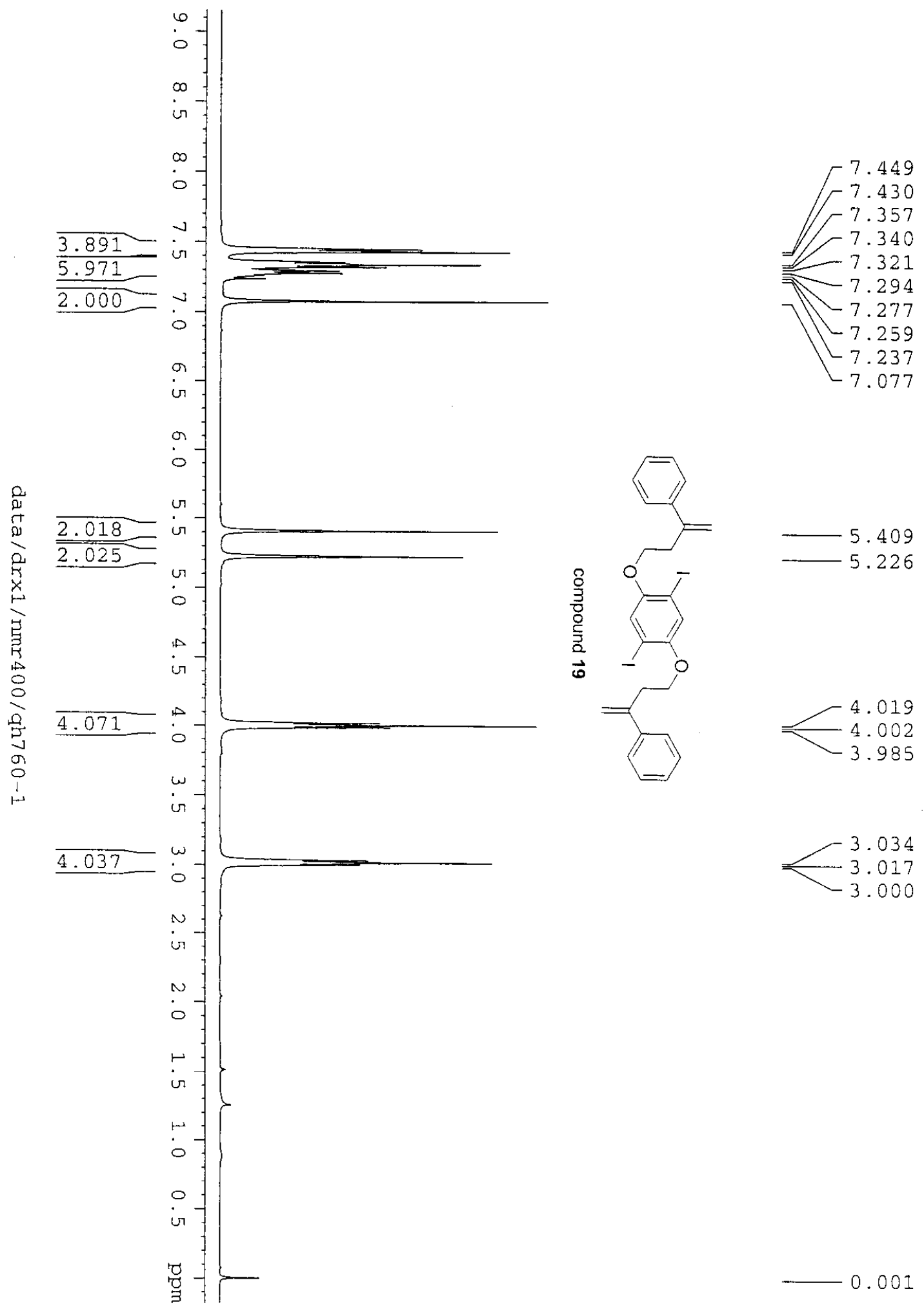


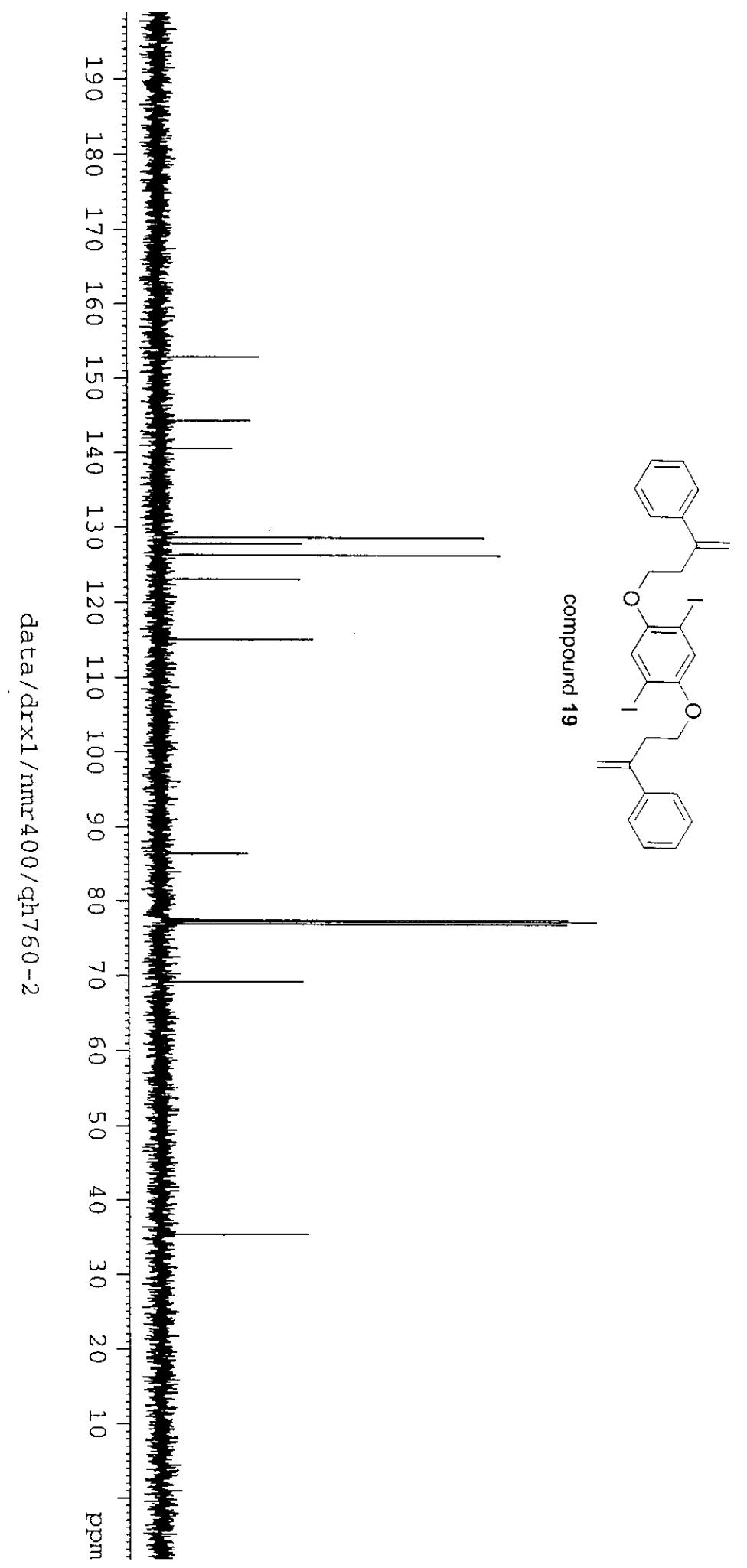

152.906

144.300

140.595
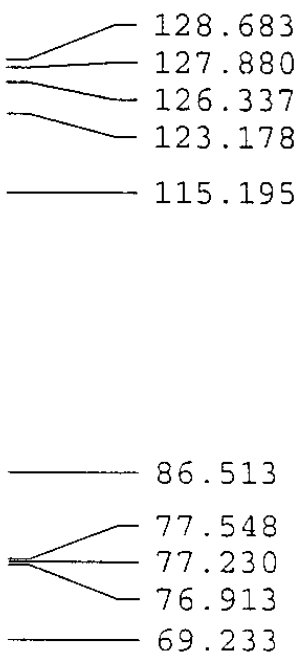

35.334 


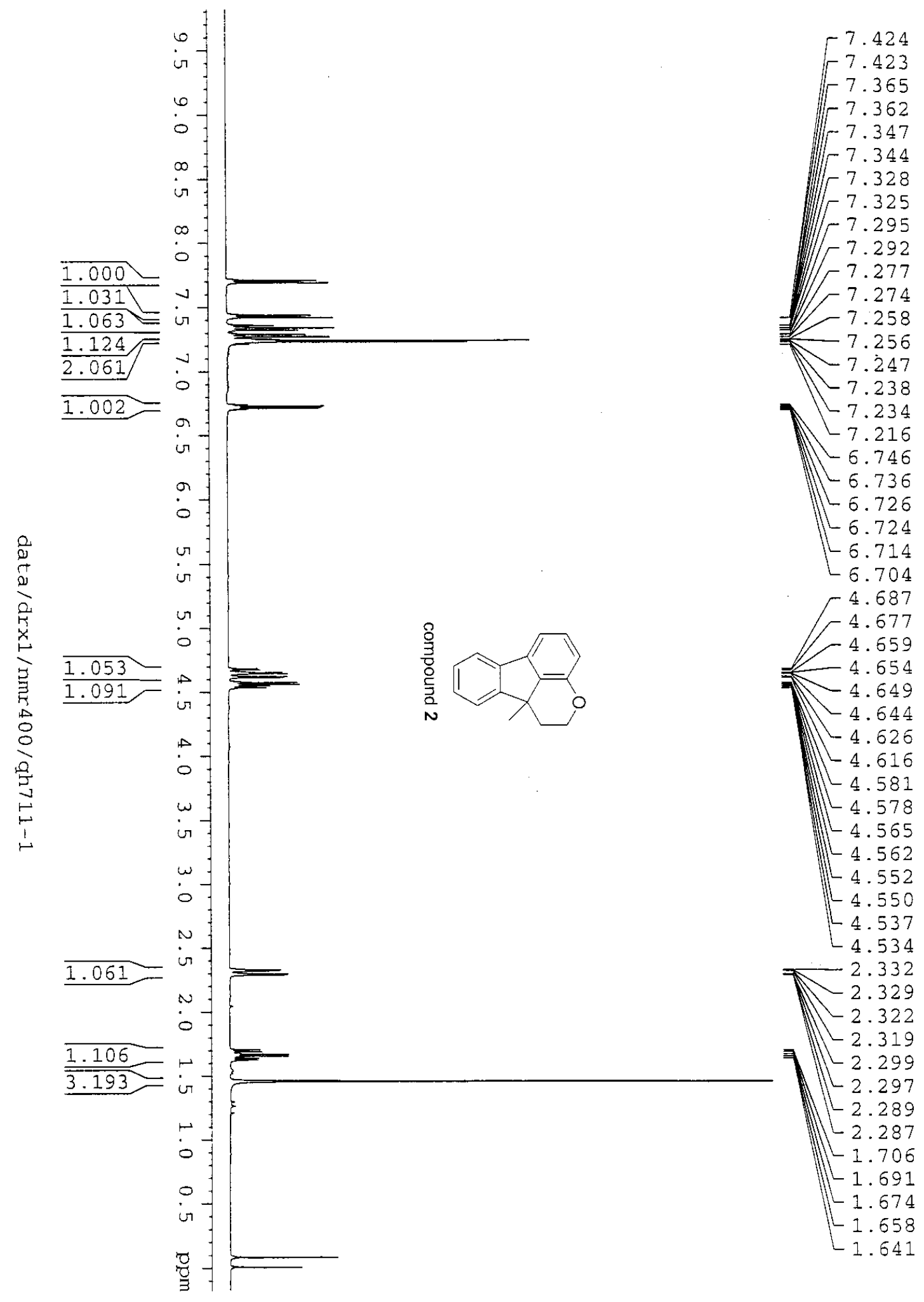



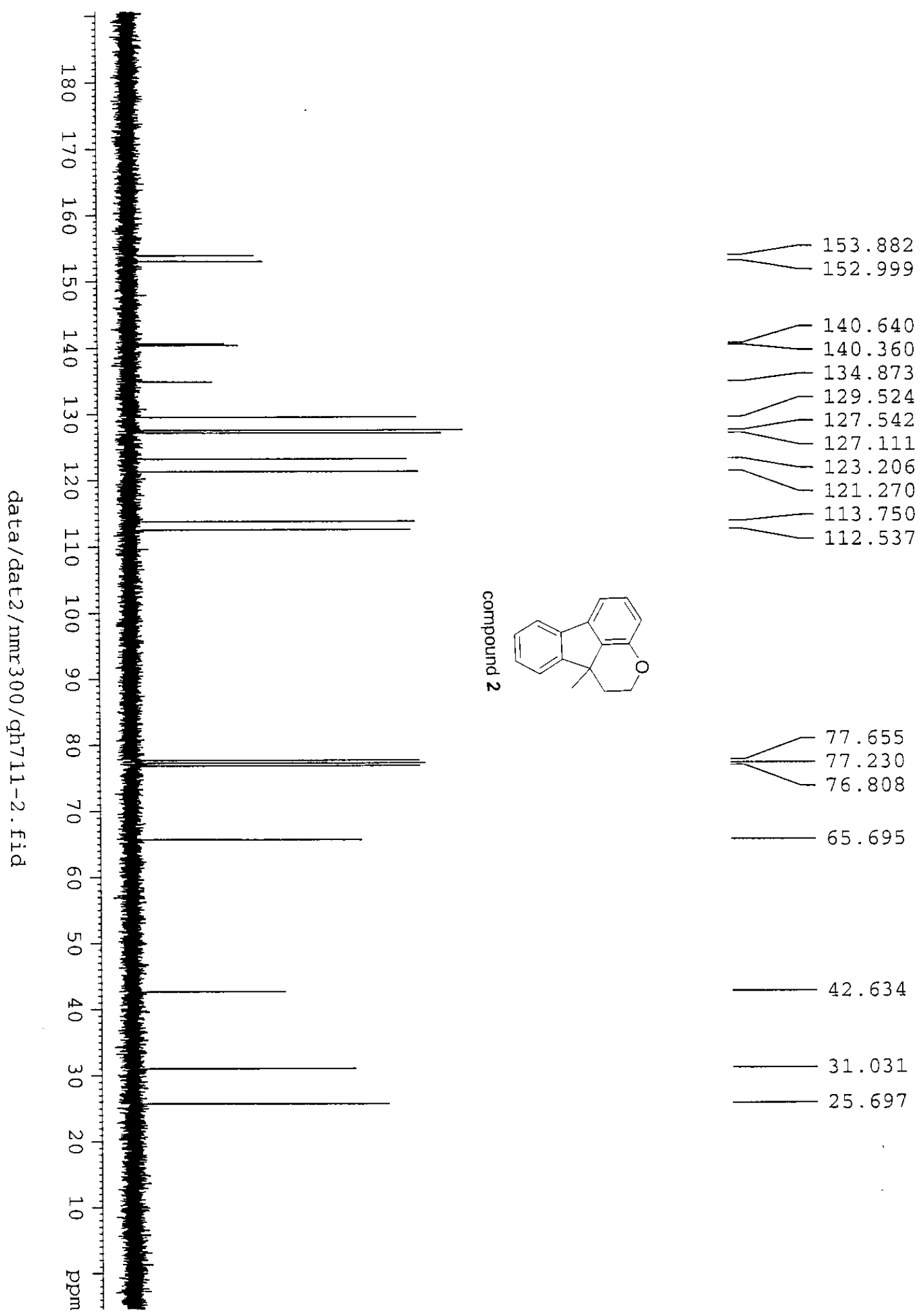

$-65.695$

42.634

31.031

25.697 


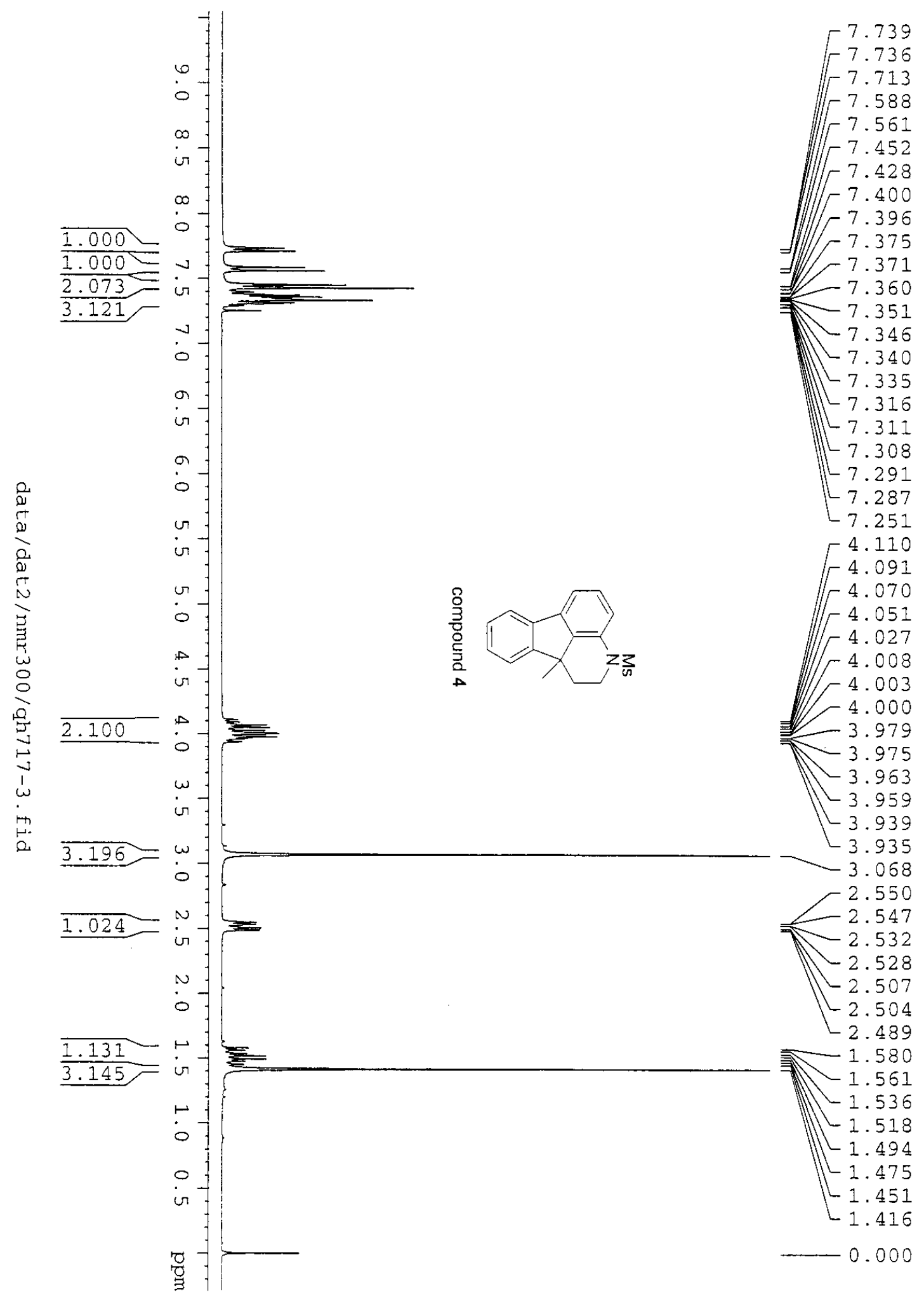



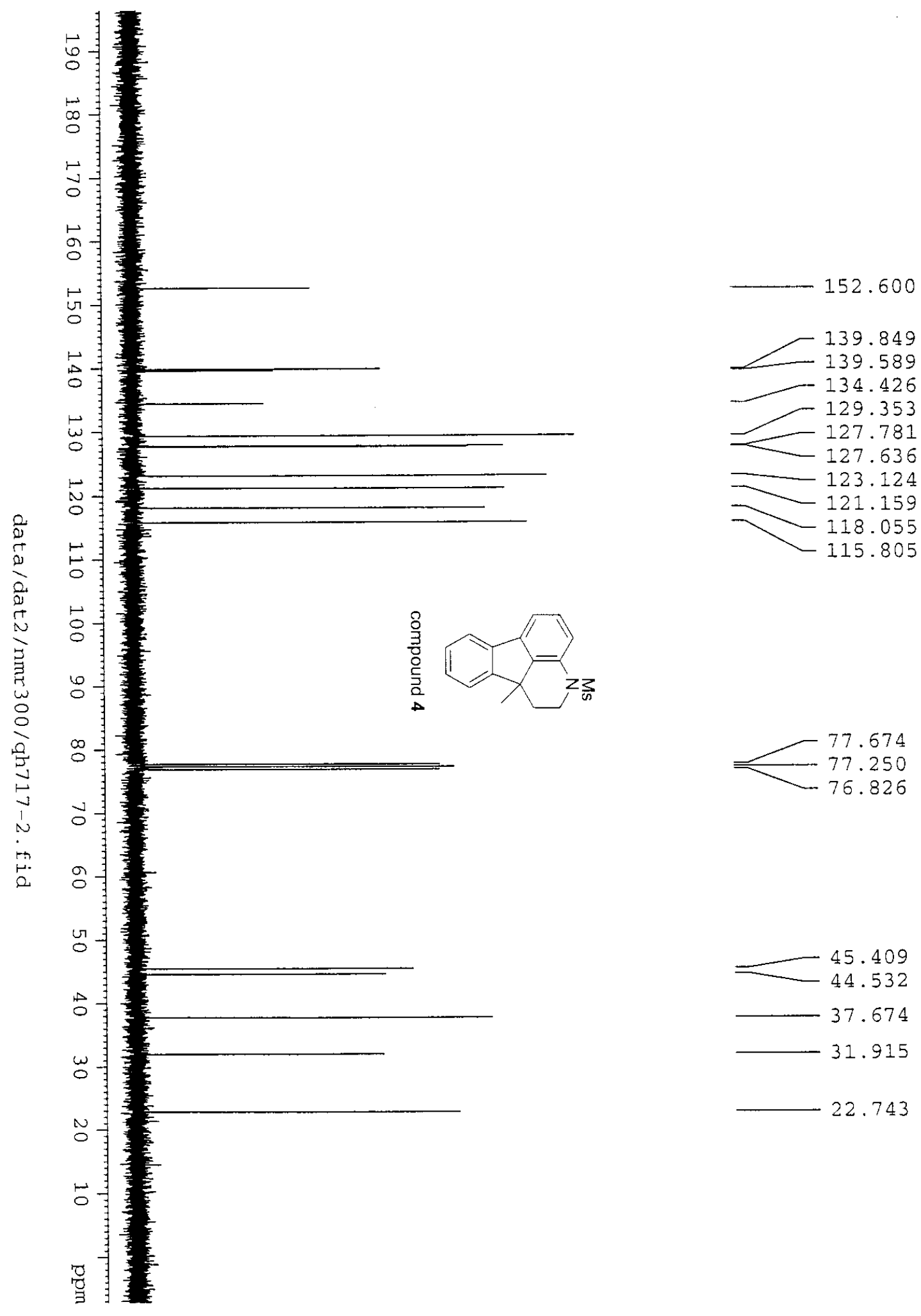

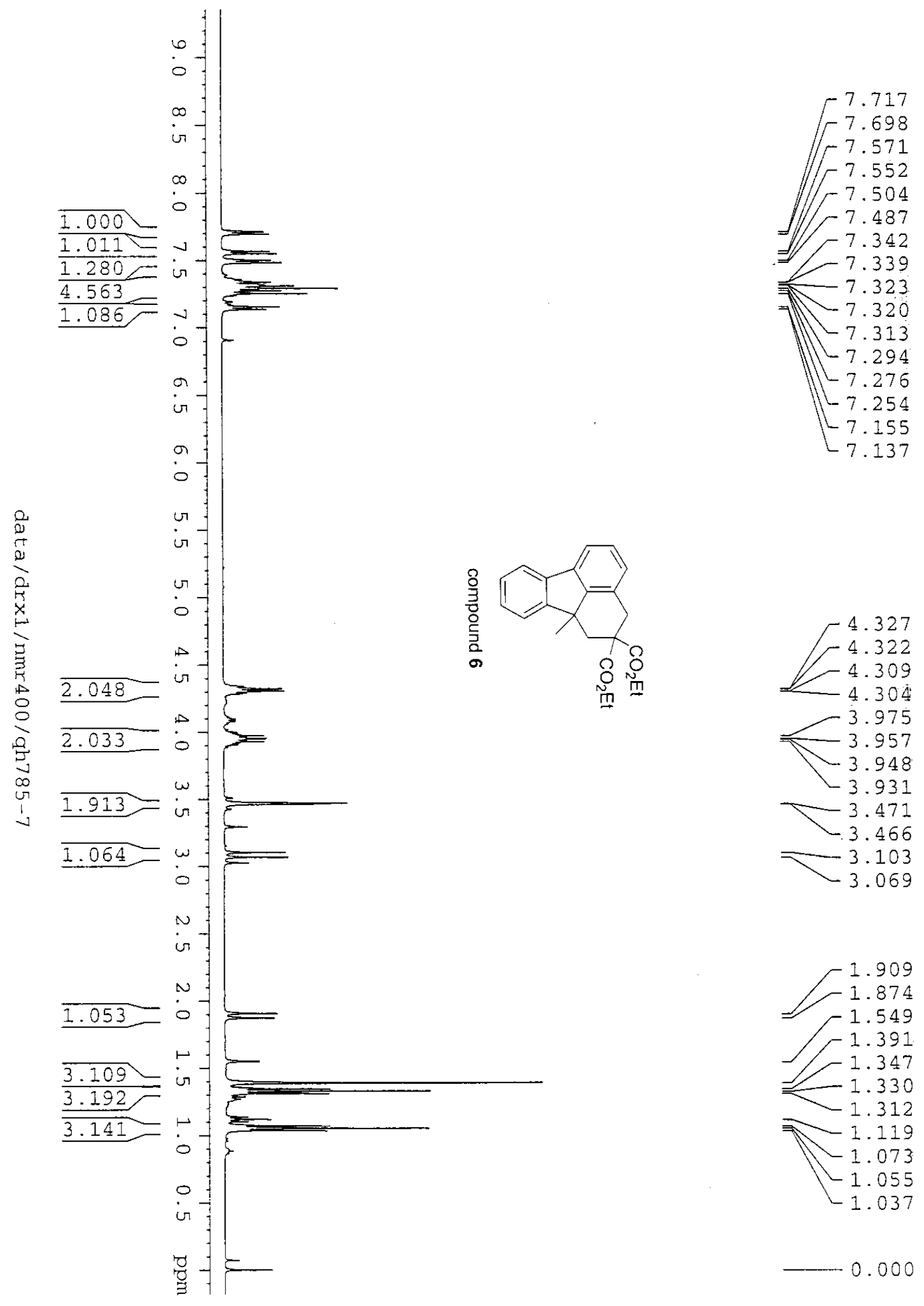

1.909
-1.874
1.549

$-1.391$

$-1.347$

$-1.330$

$-1.312$

$\approx-1.119$

$-1.073$

$-1.055$

$-1.037$

0.000 


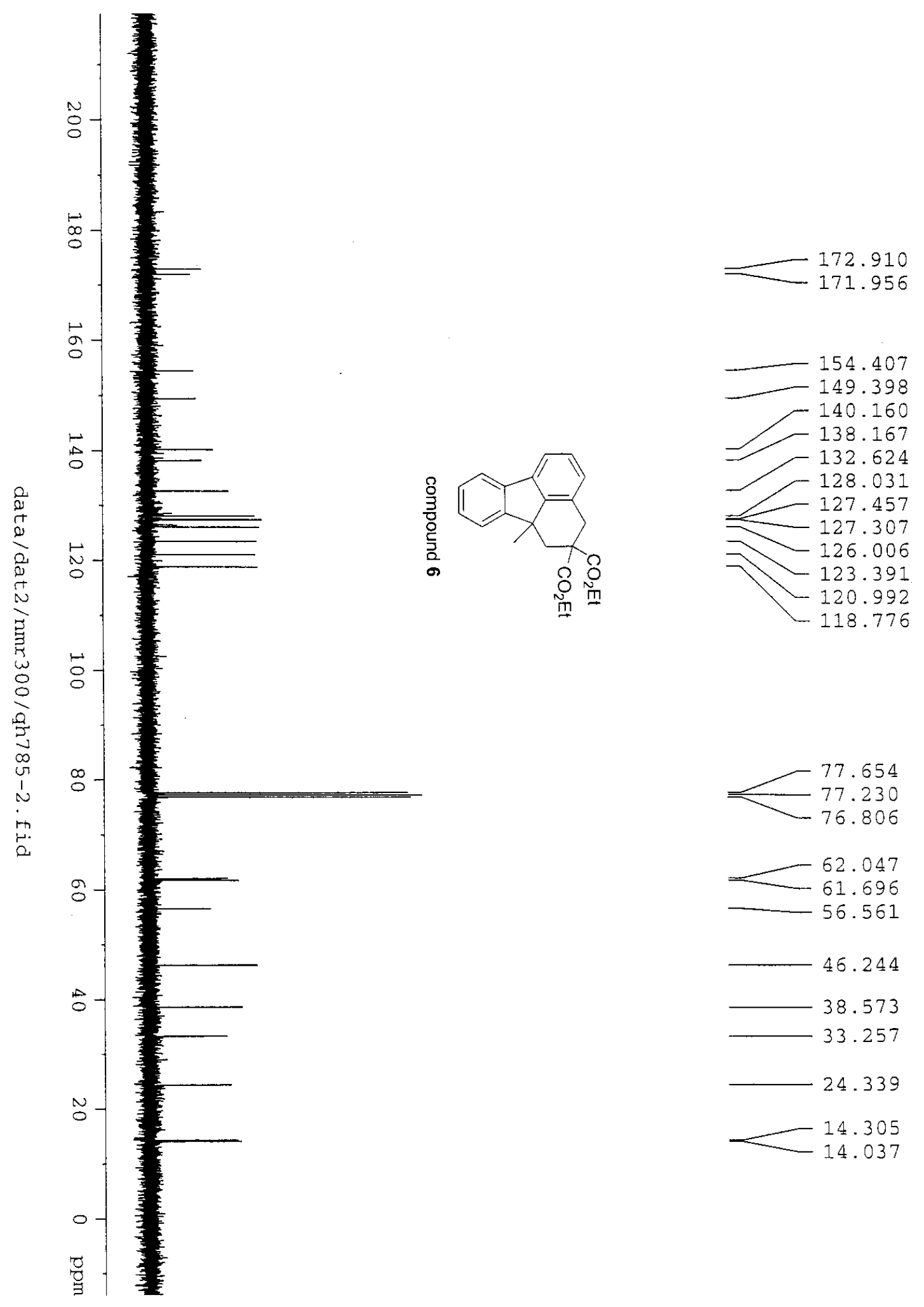




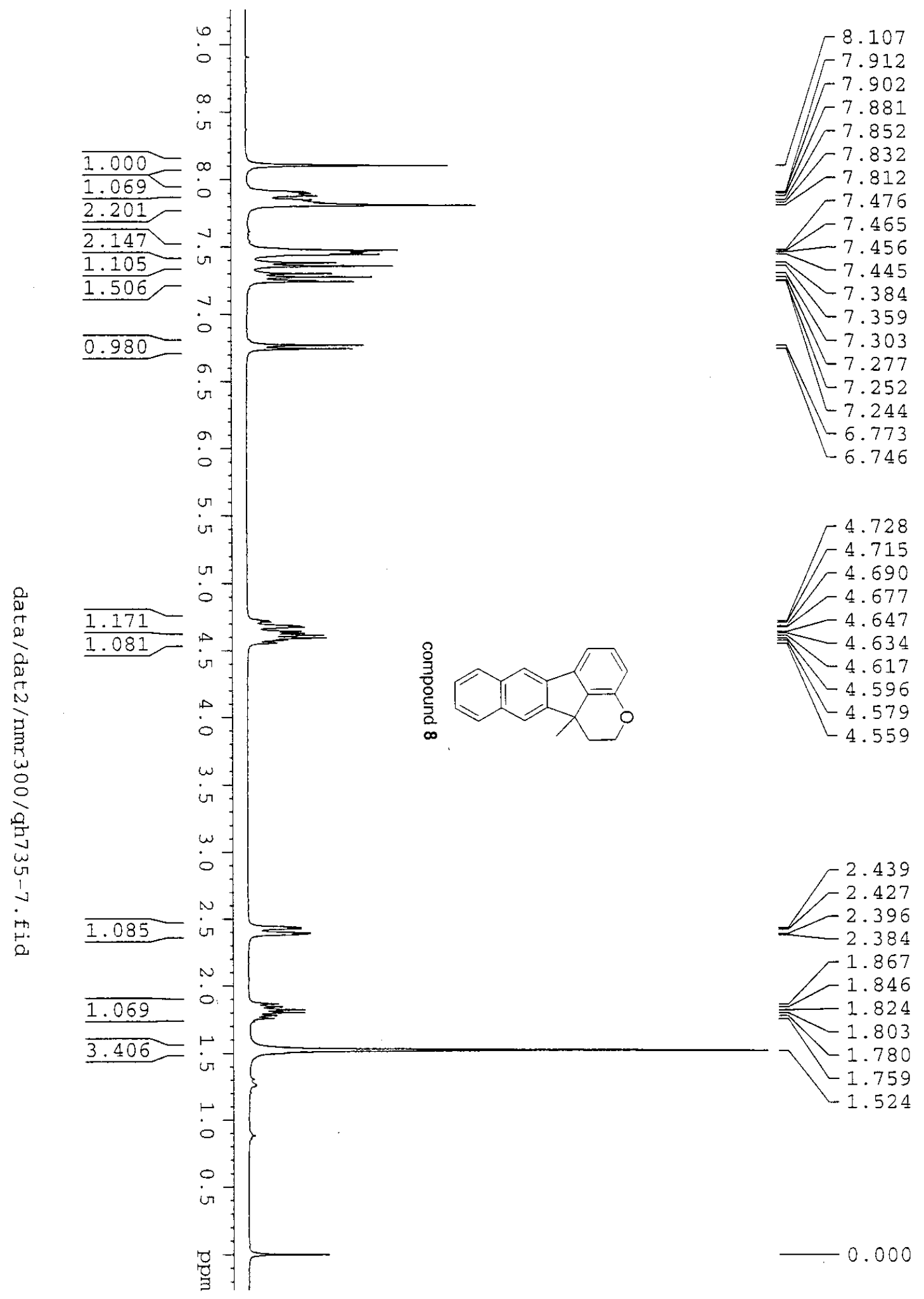




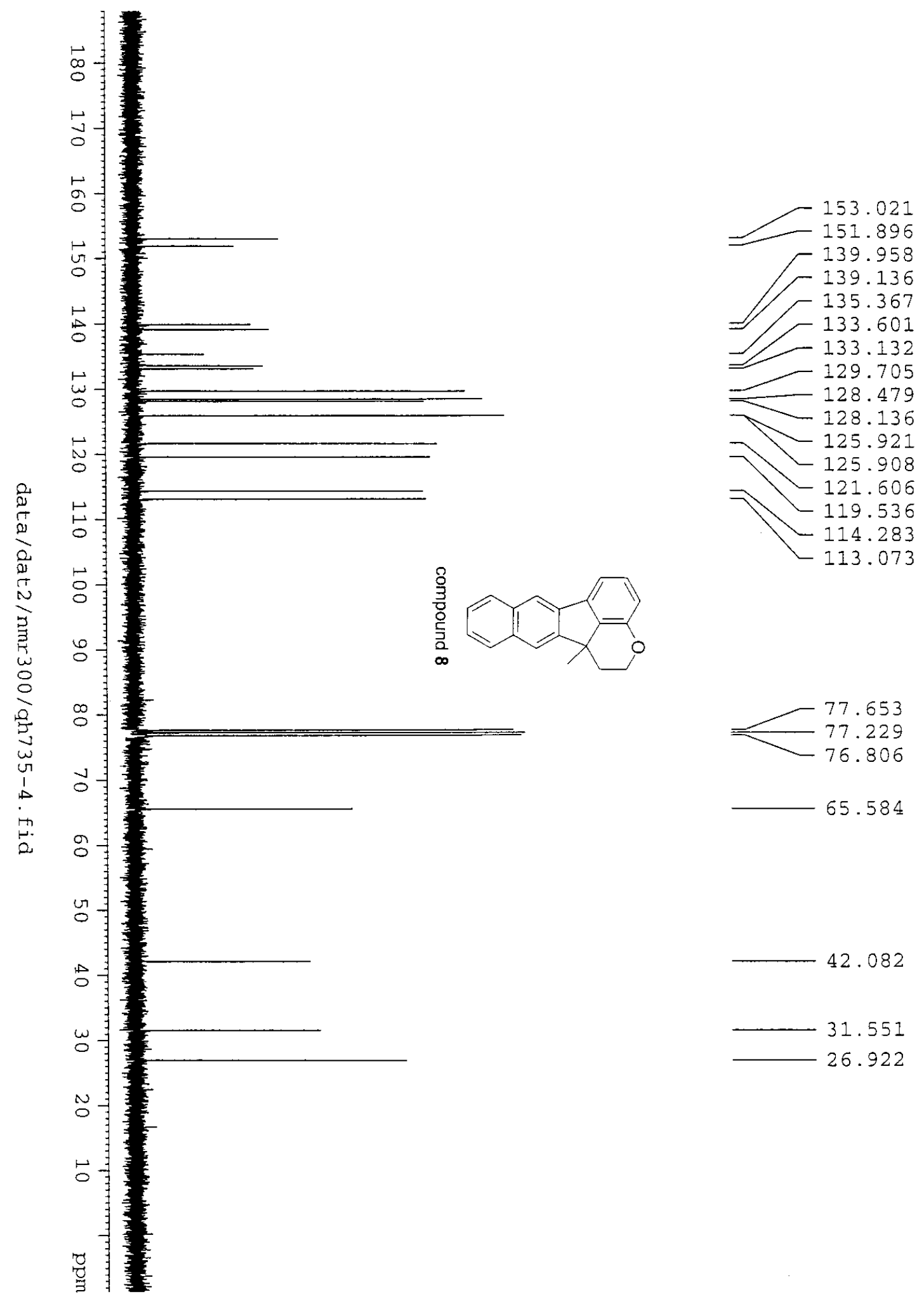




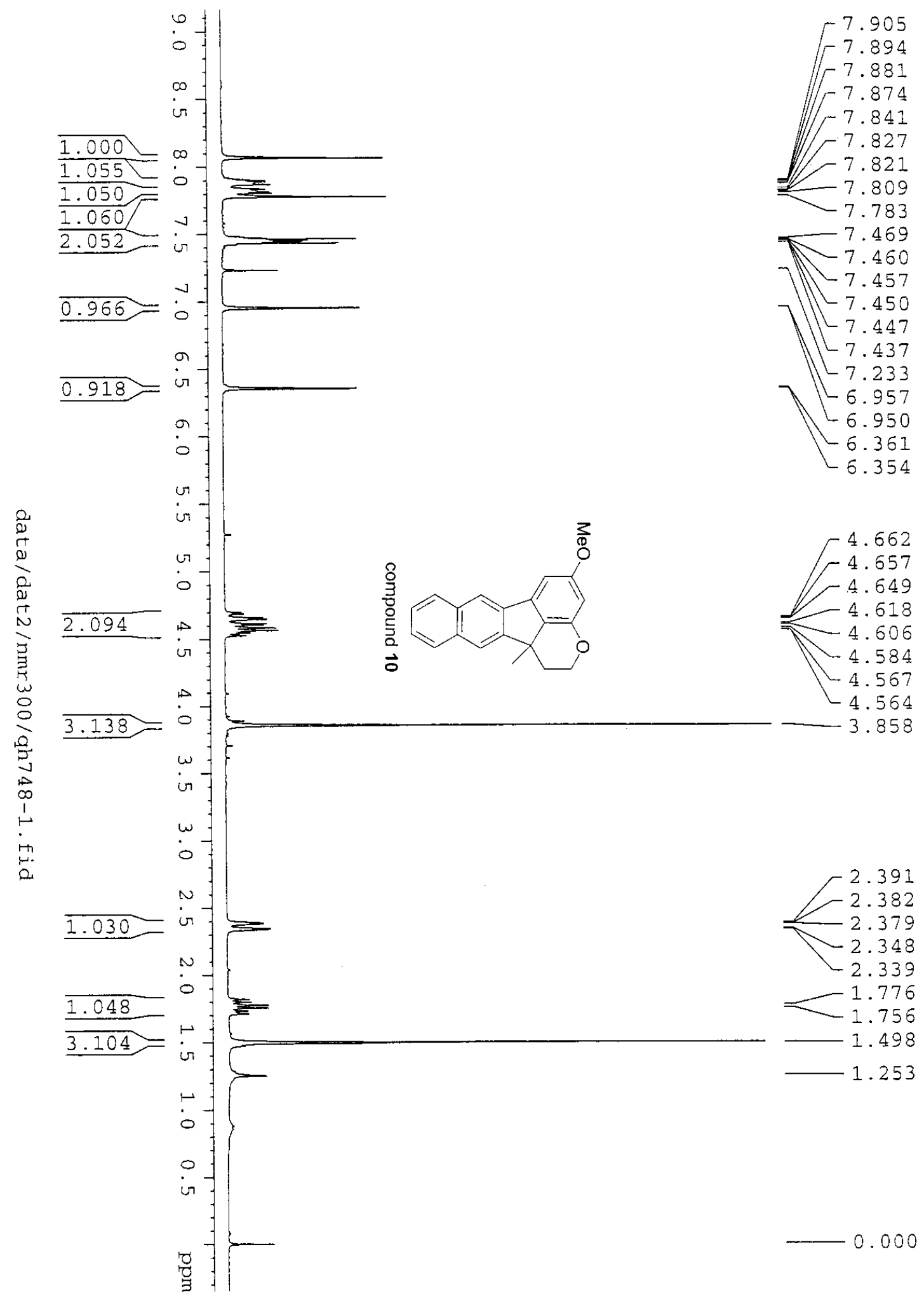




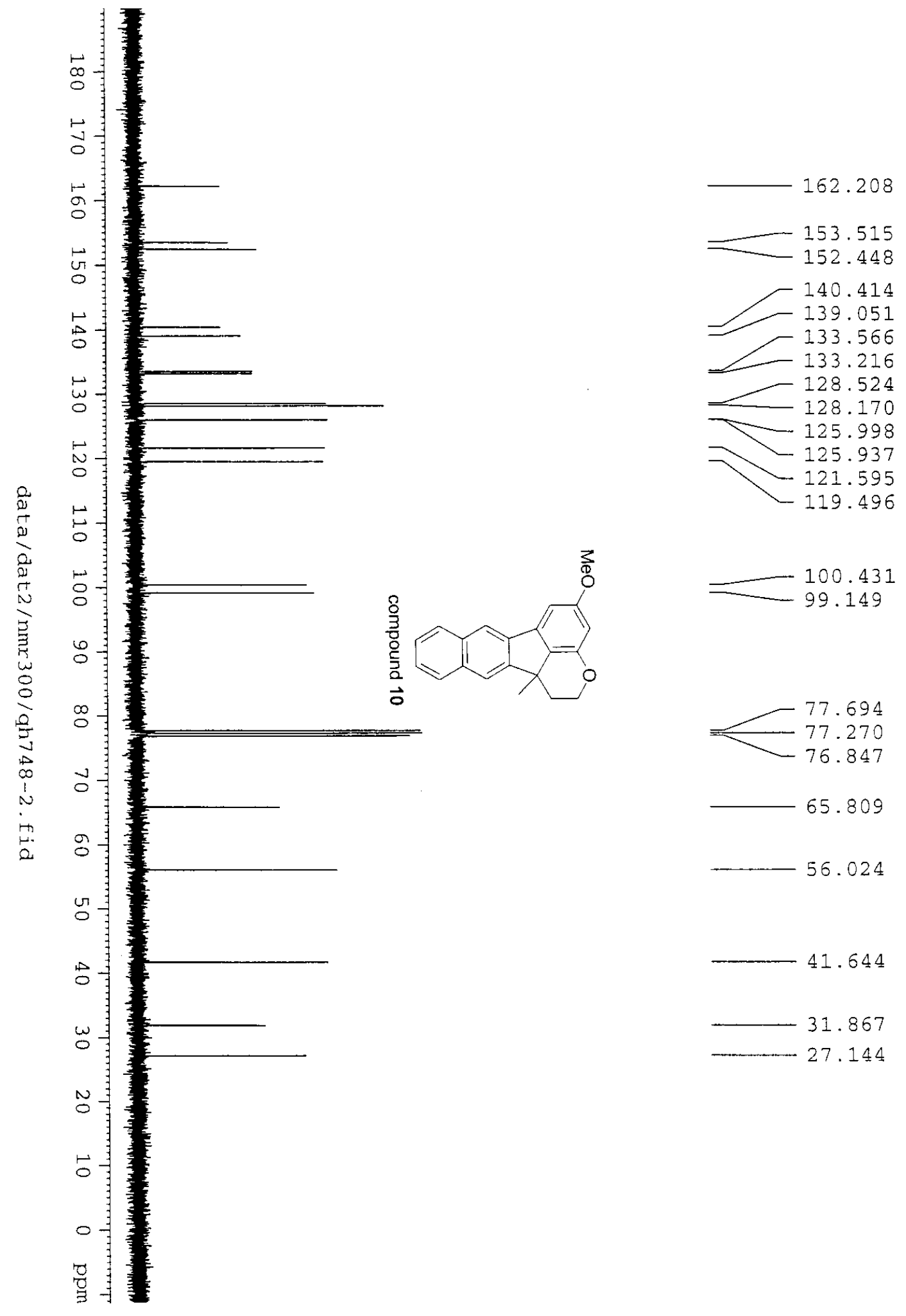



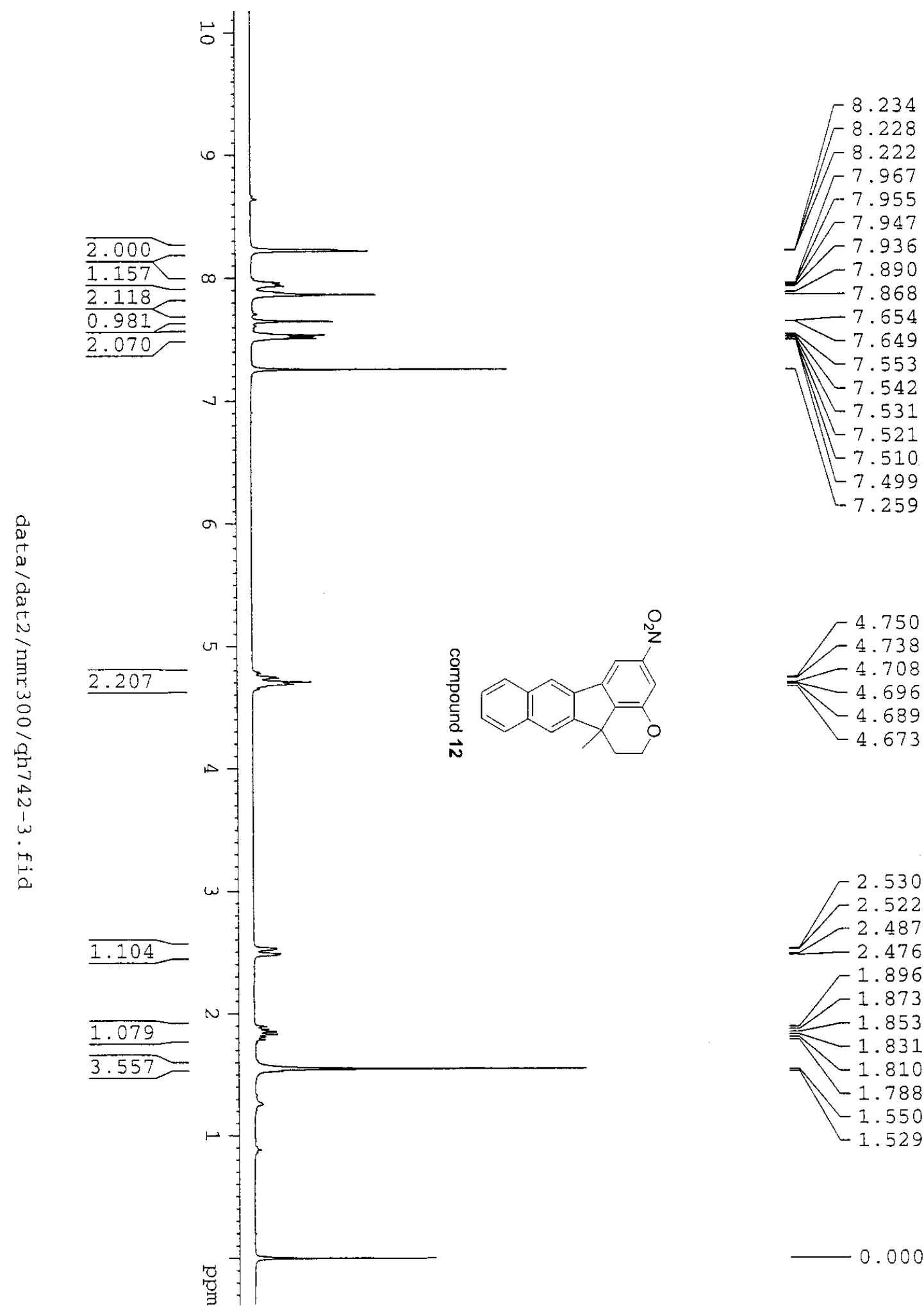
1.896 1.873 1.853

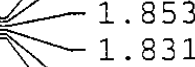

$-1.810$

$-1.788$

$-1.550$

$-1.529$

0.000 


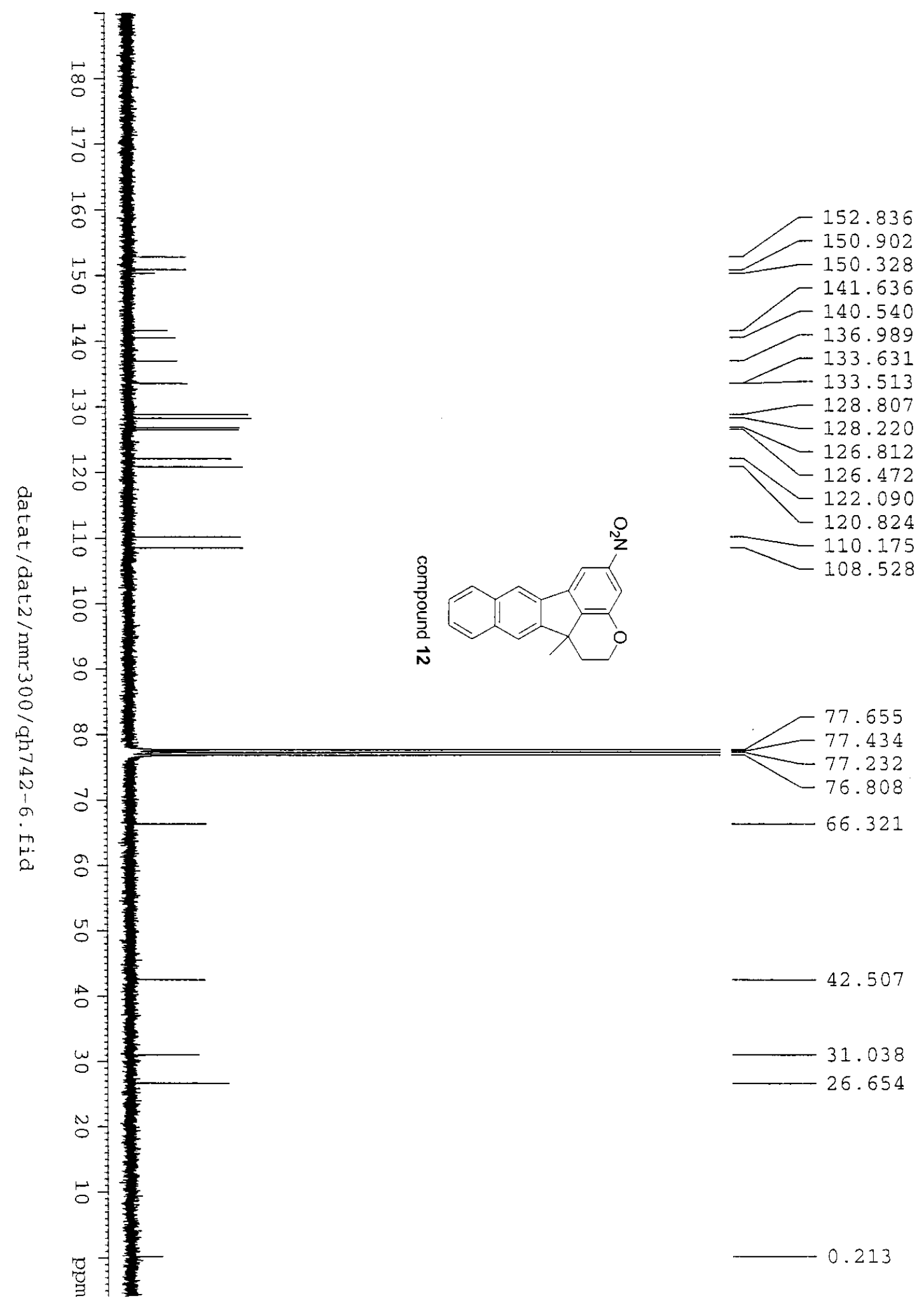




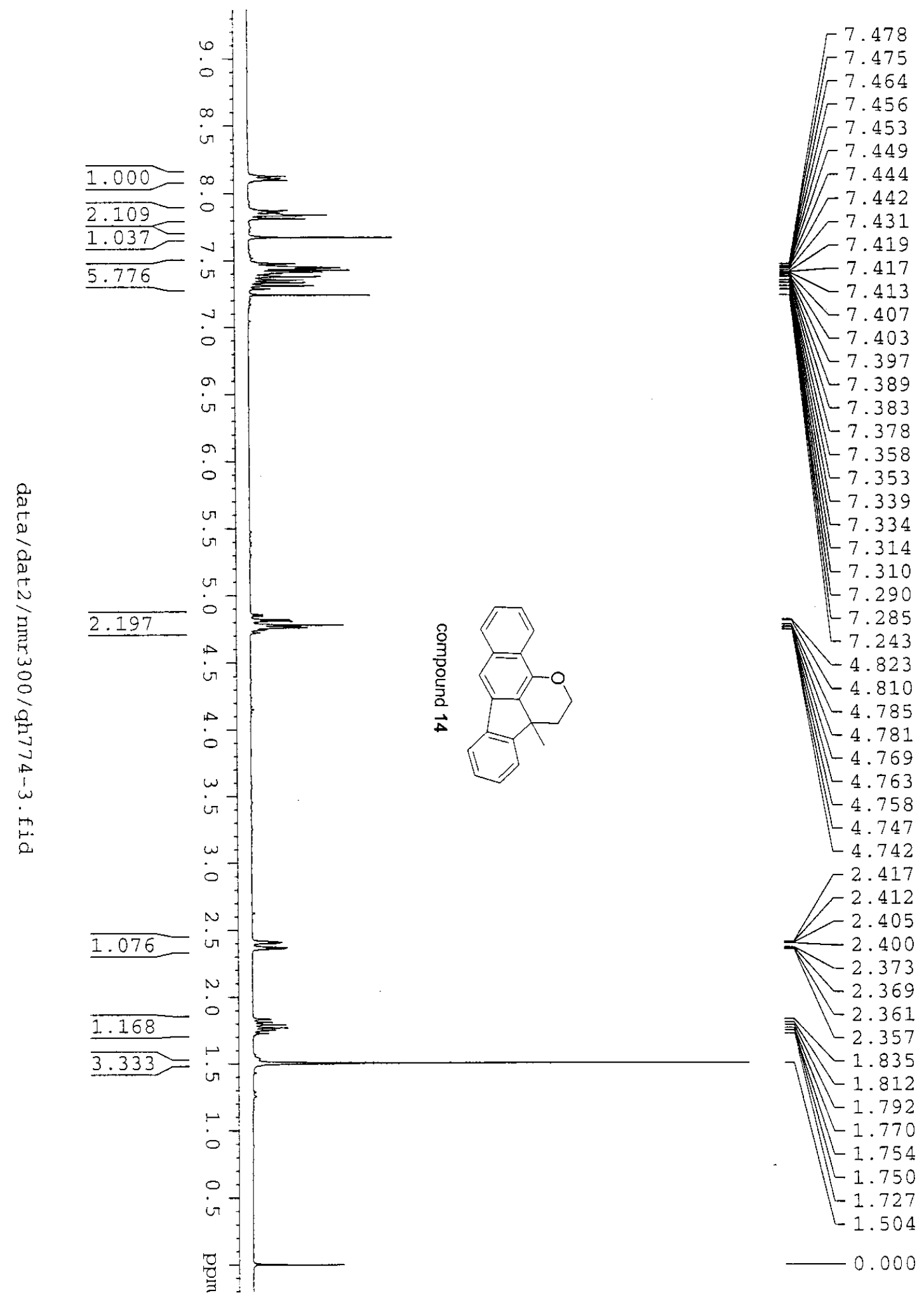




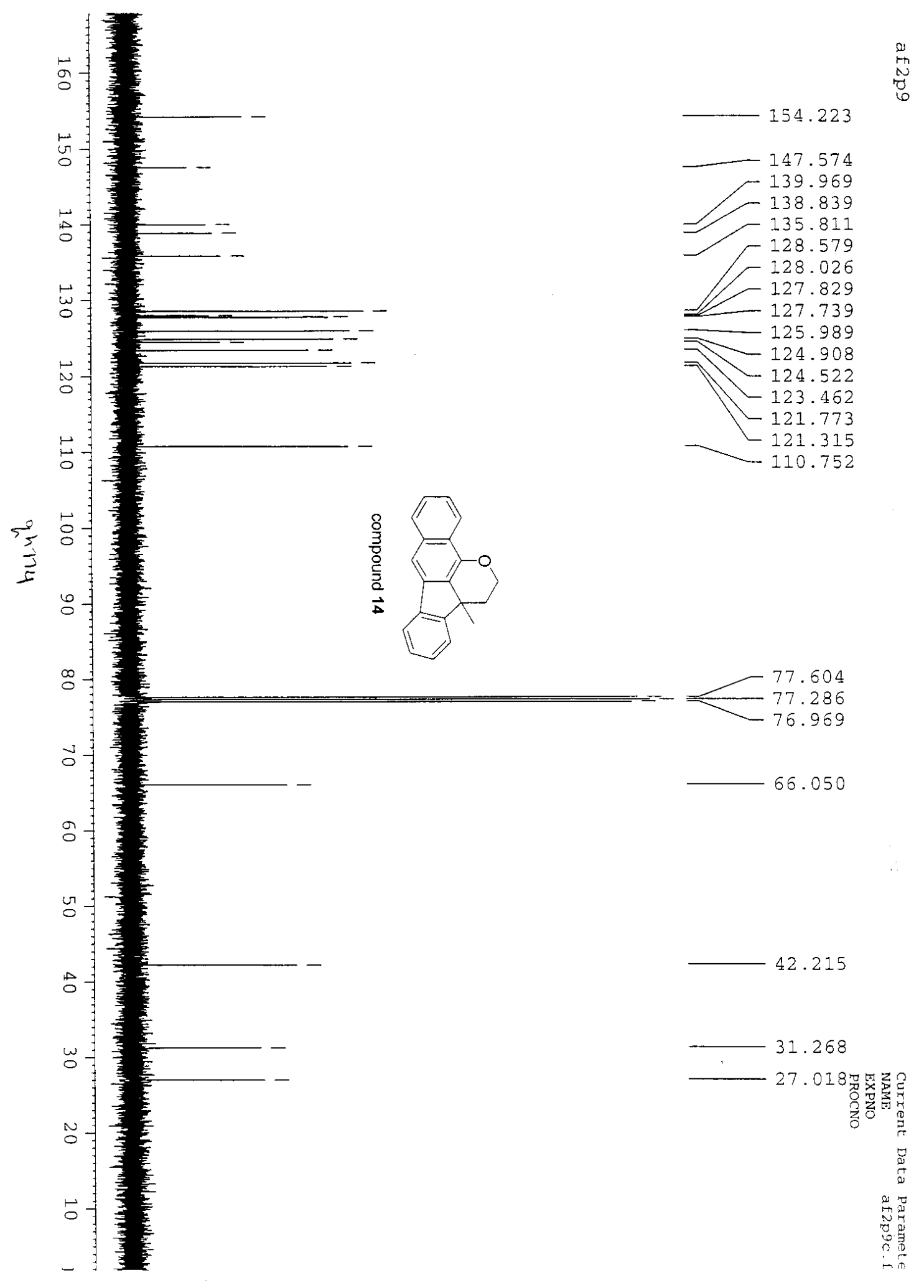



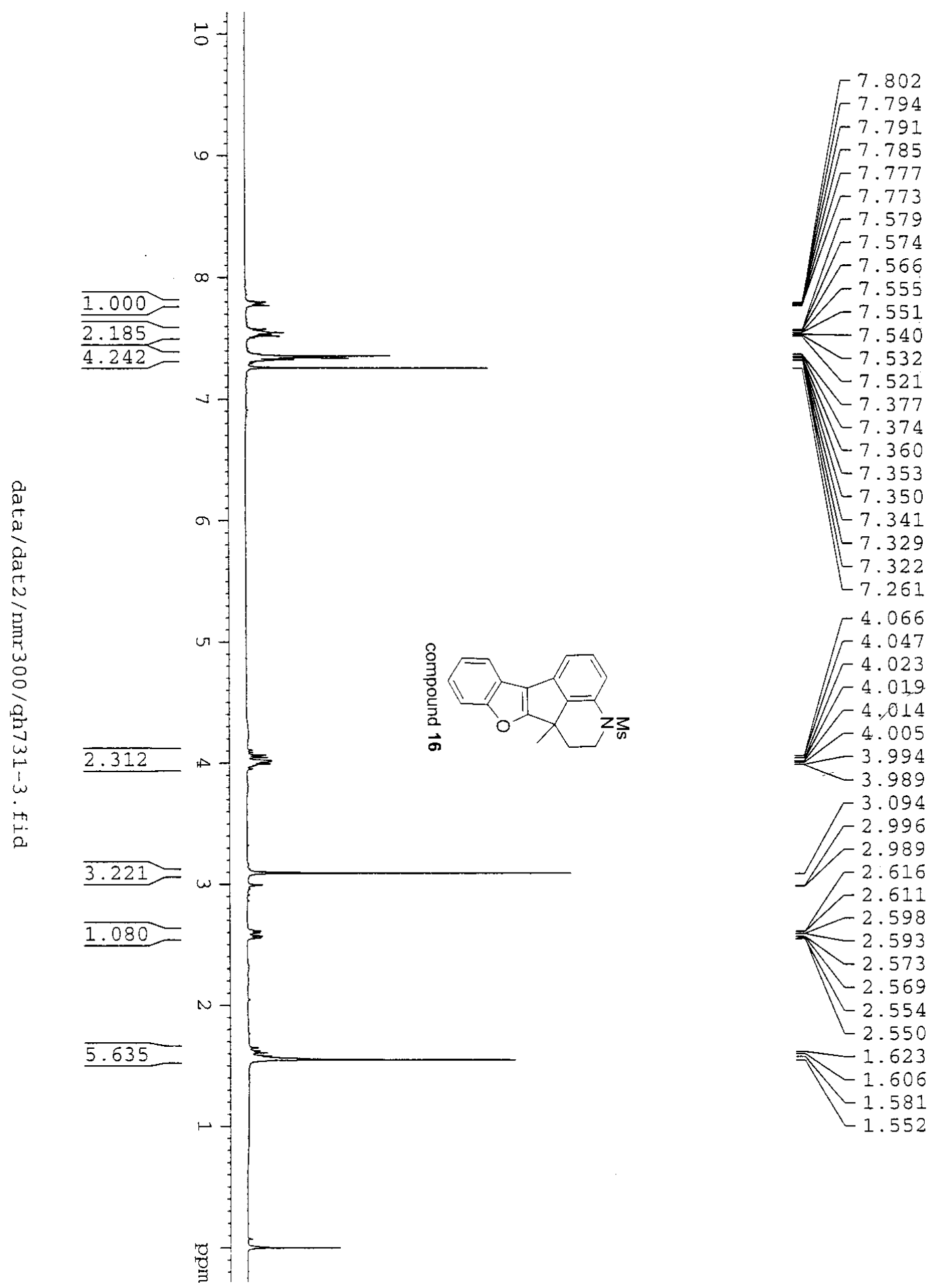


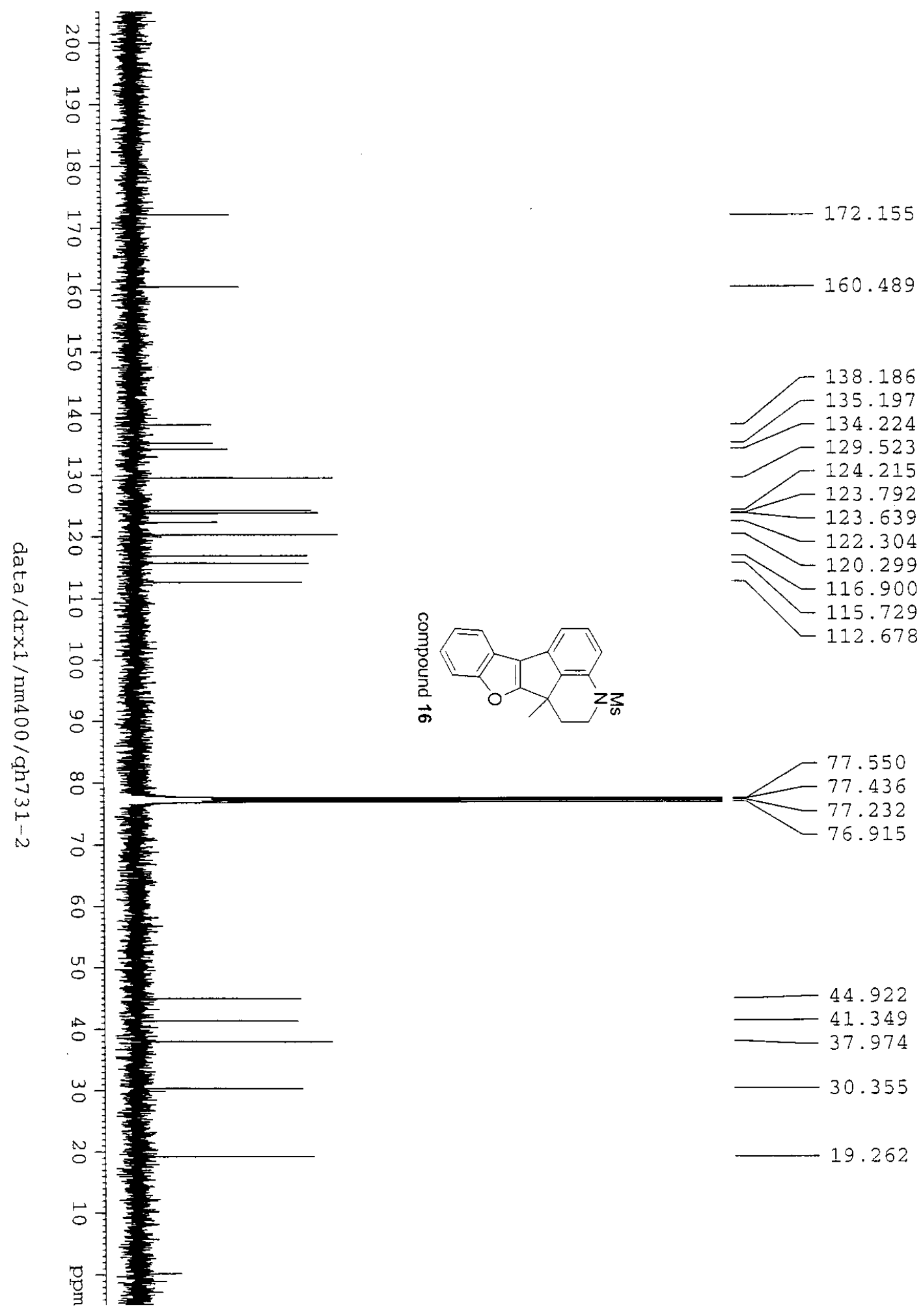




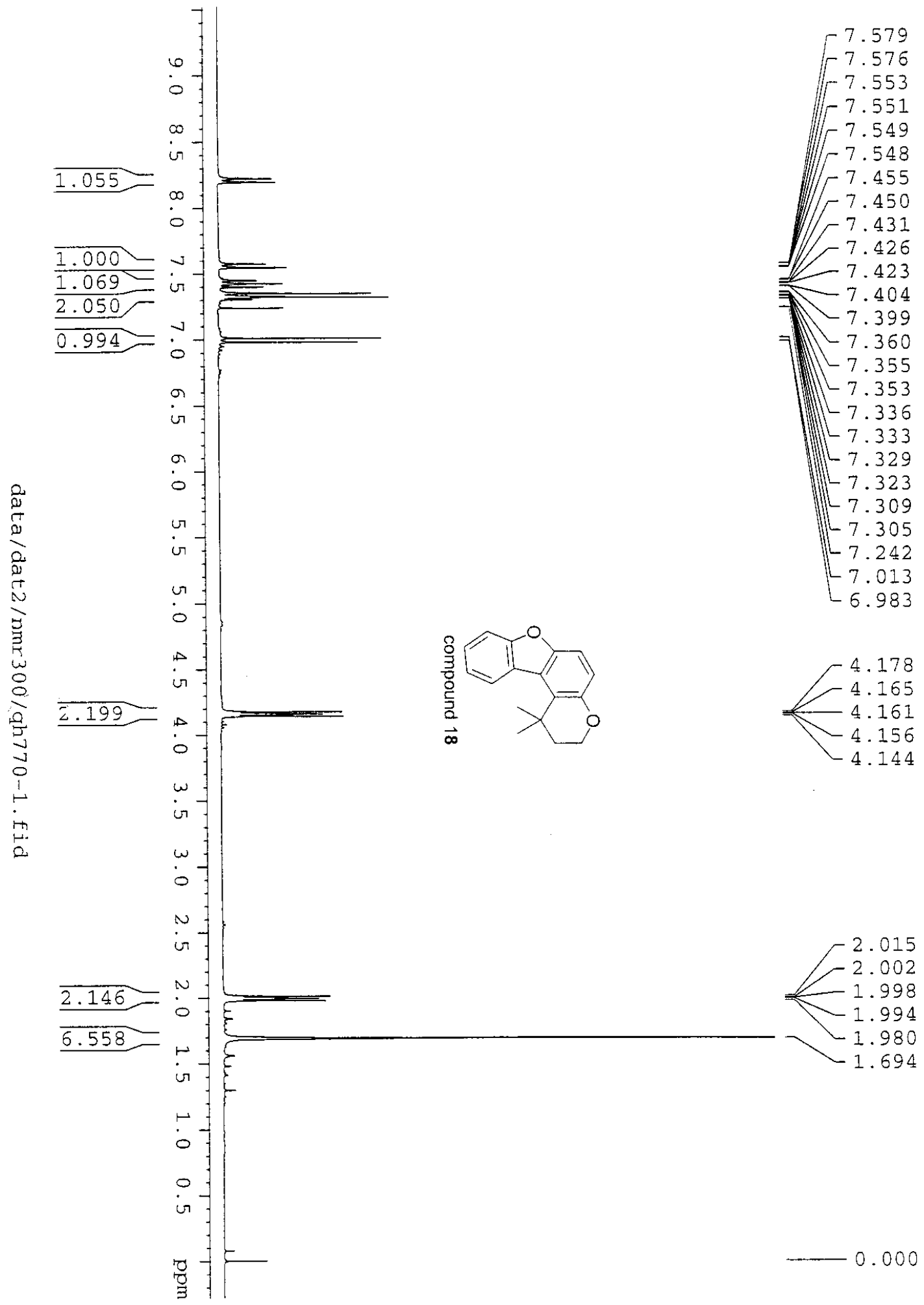



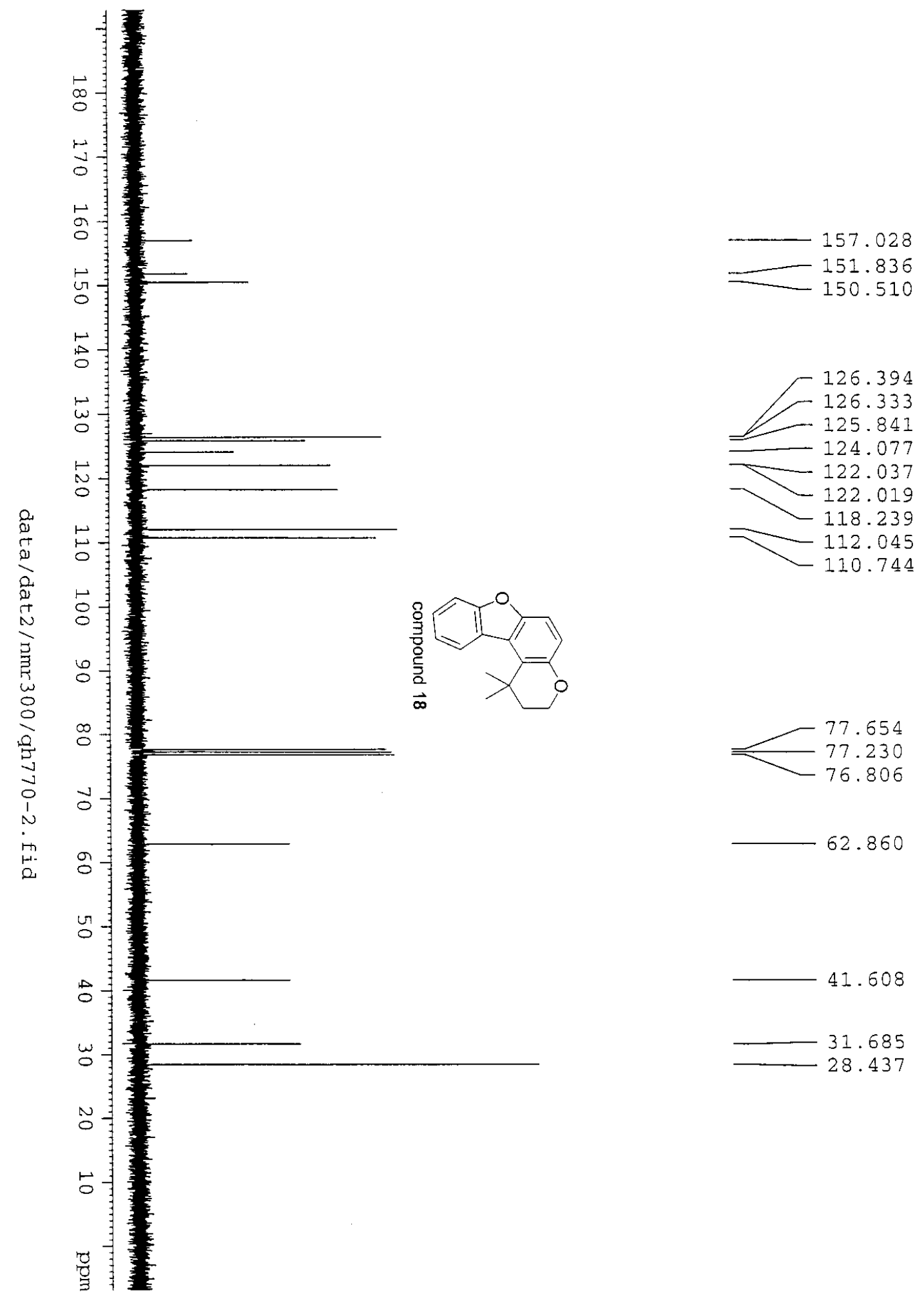

62.860

41.608

31.685 28.437 

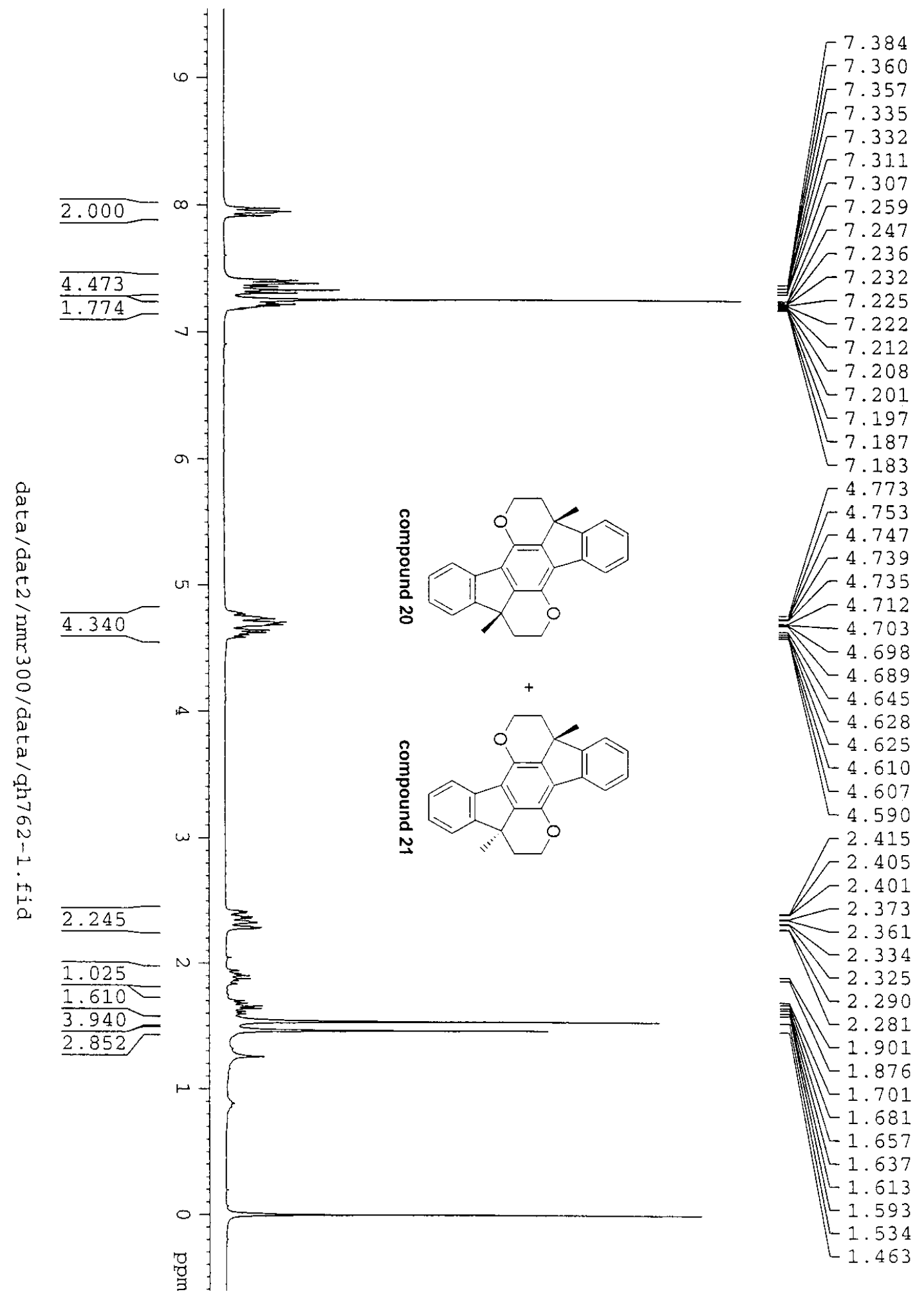


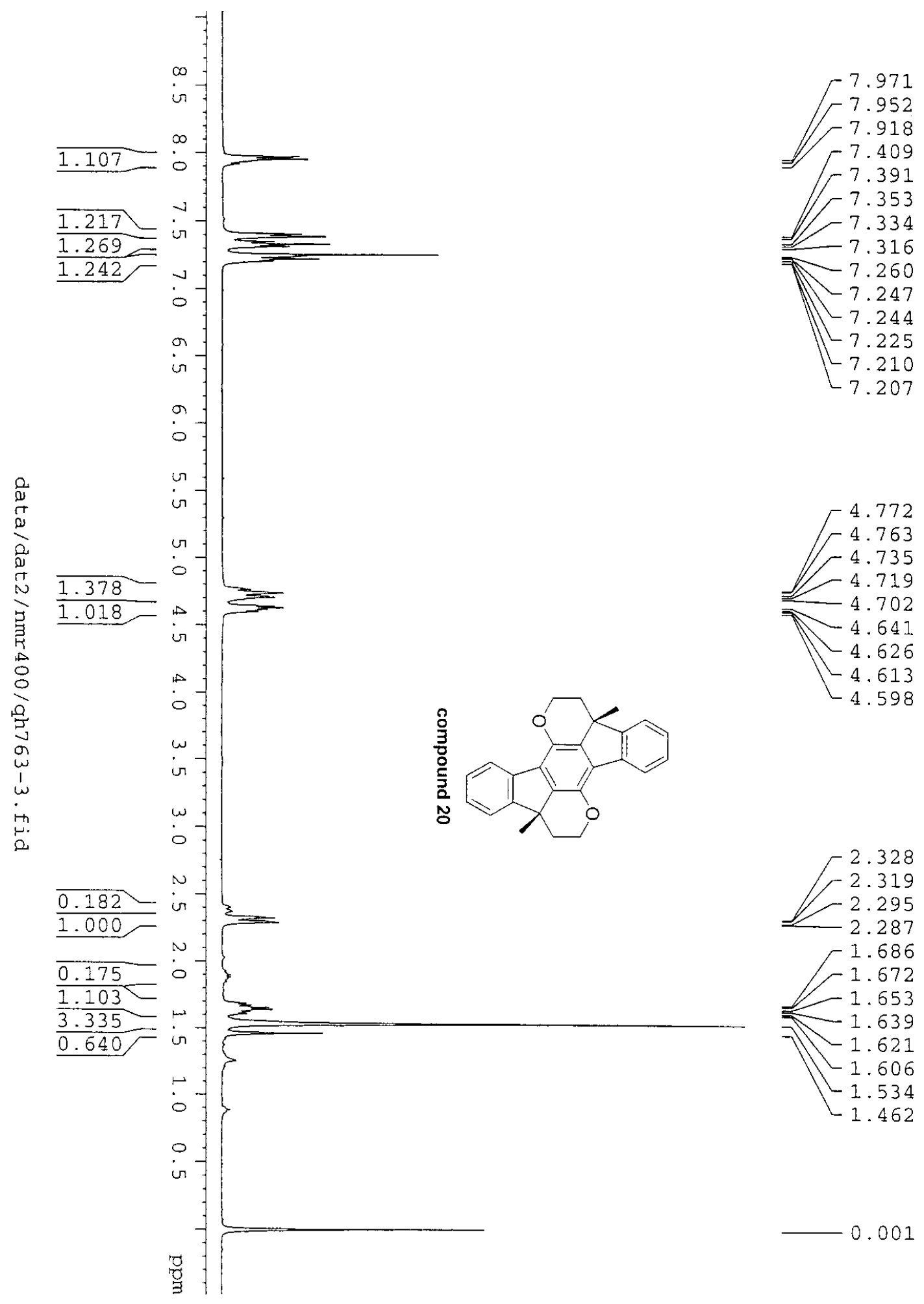




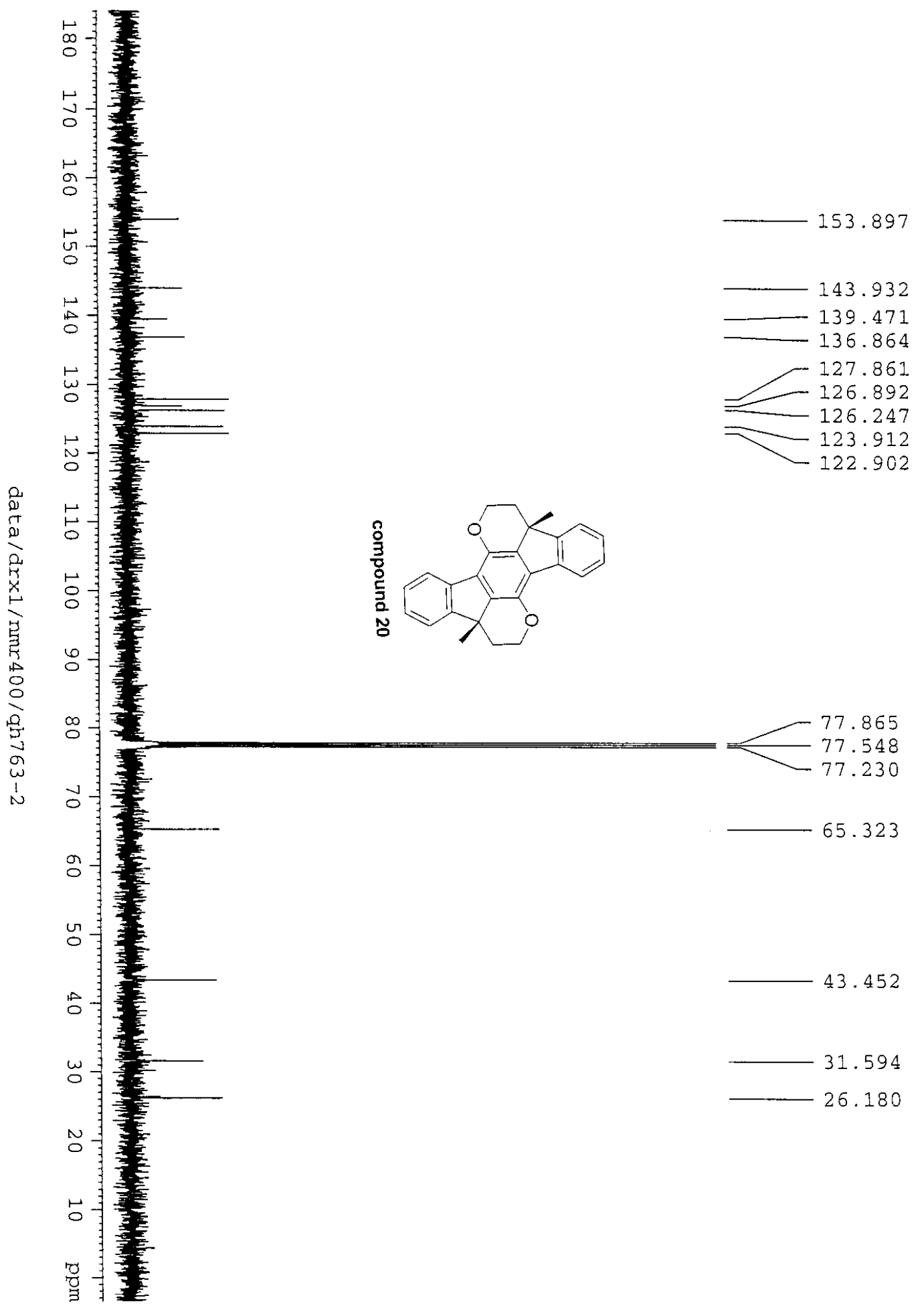



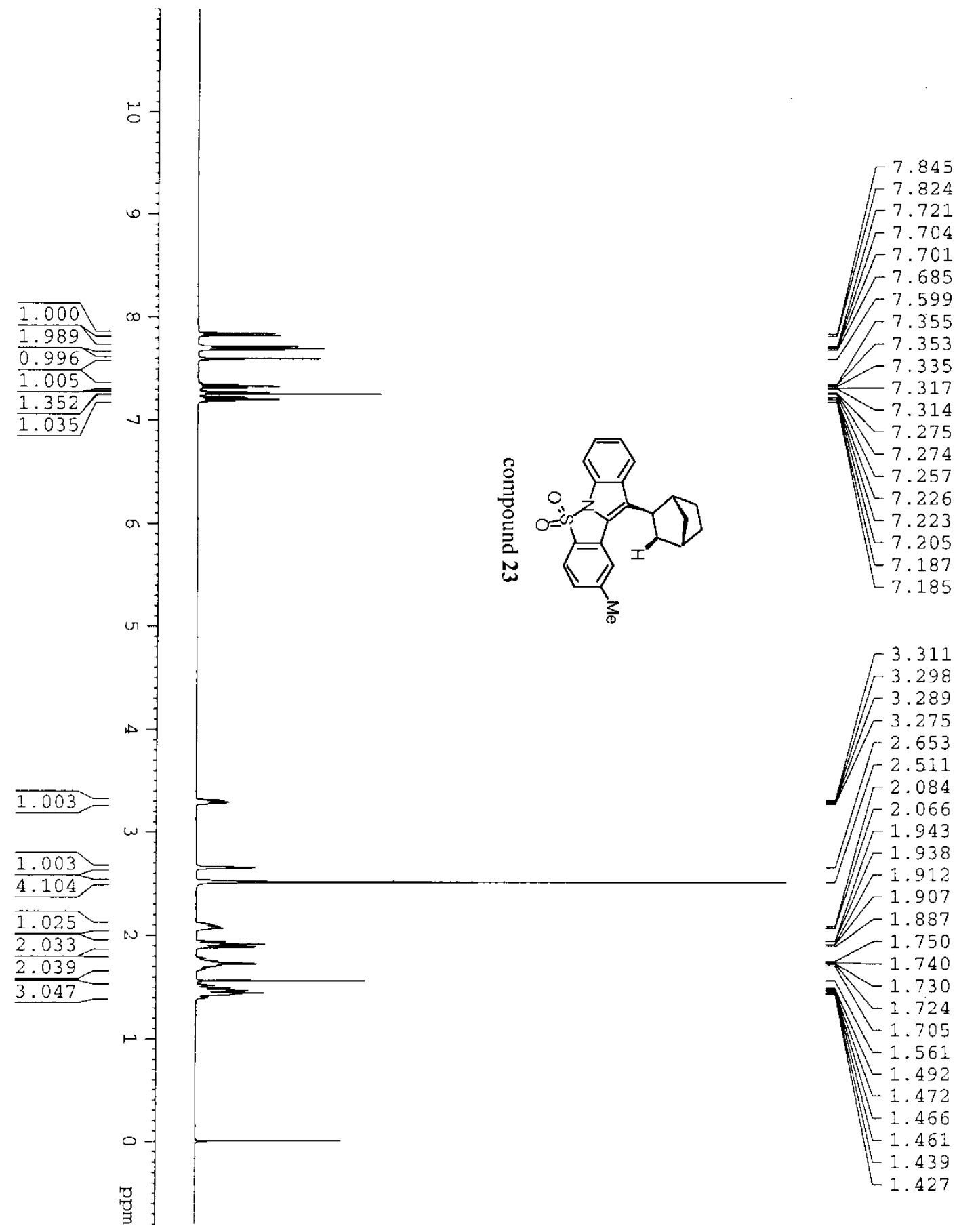

III 2.653

/II 2.511

$\int \Gamma^{2} .084$

/ -2.066

II 1.943

$-1.938$

]$/ 1.912$

(t -1.907

III -1.887

$-1.750$

rom 1.740

$7-1.730$

$M-1.724$

$-1.705$

$-1.561$

$-1.492$

$-1.466$

1.461

1.439

$-1.427$ 


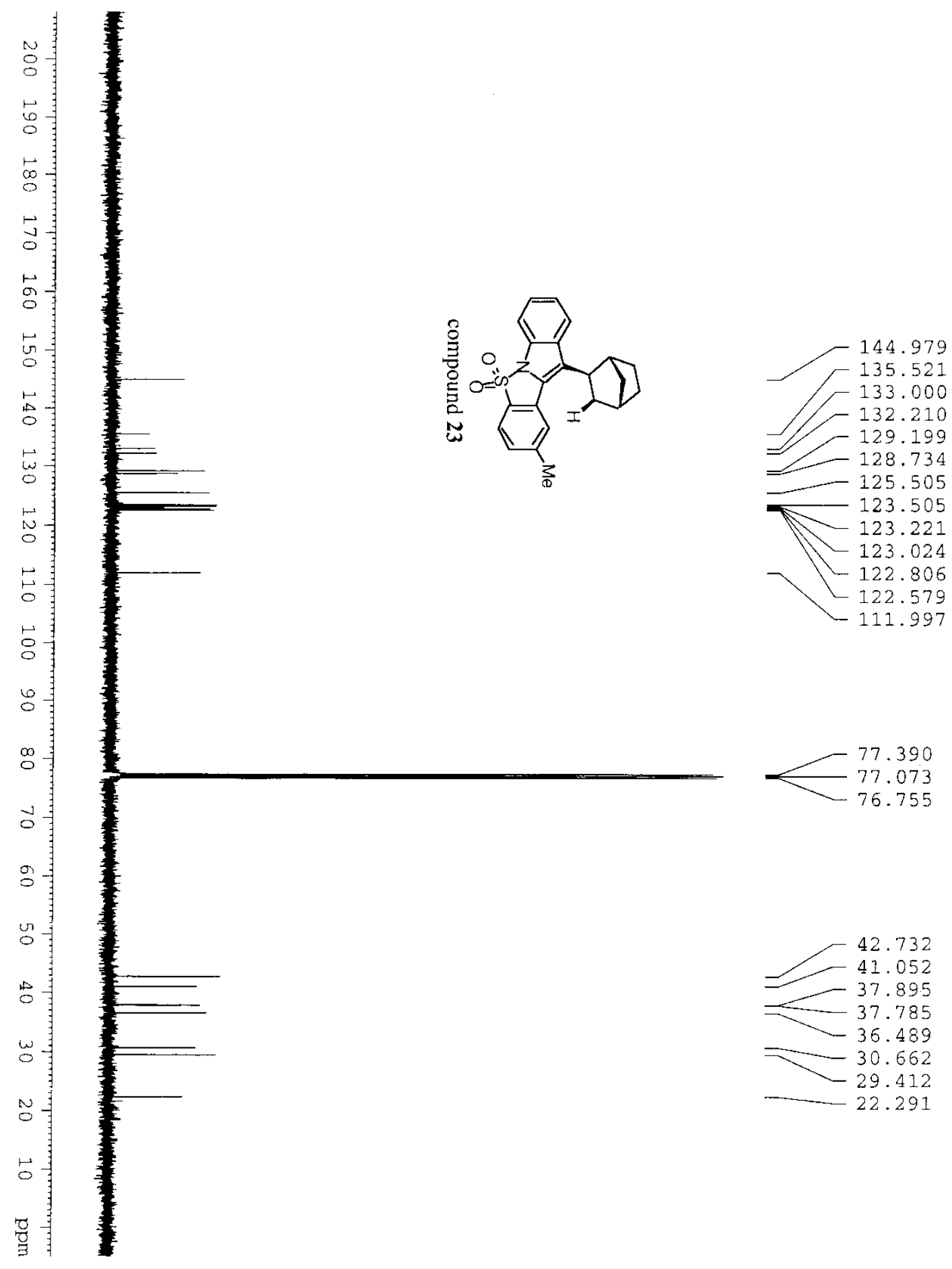



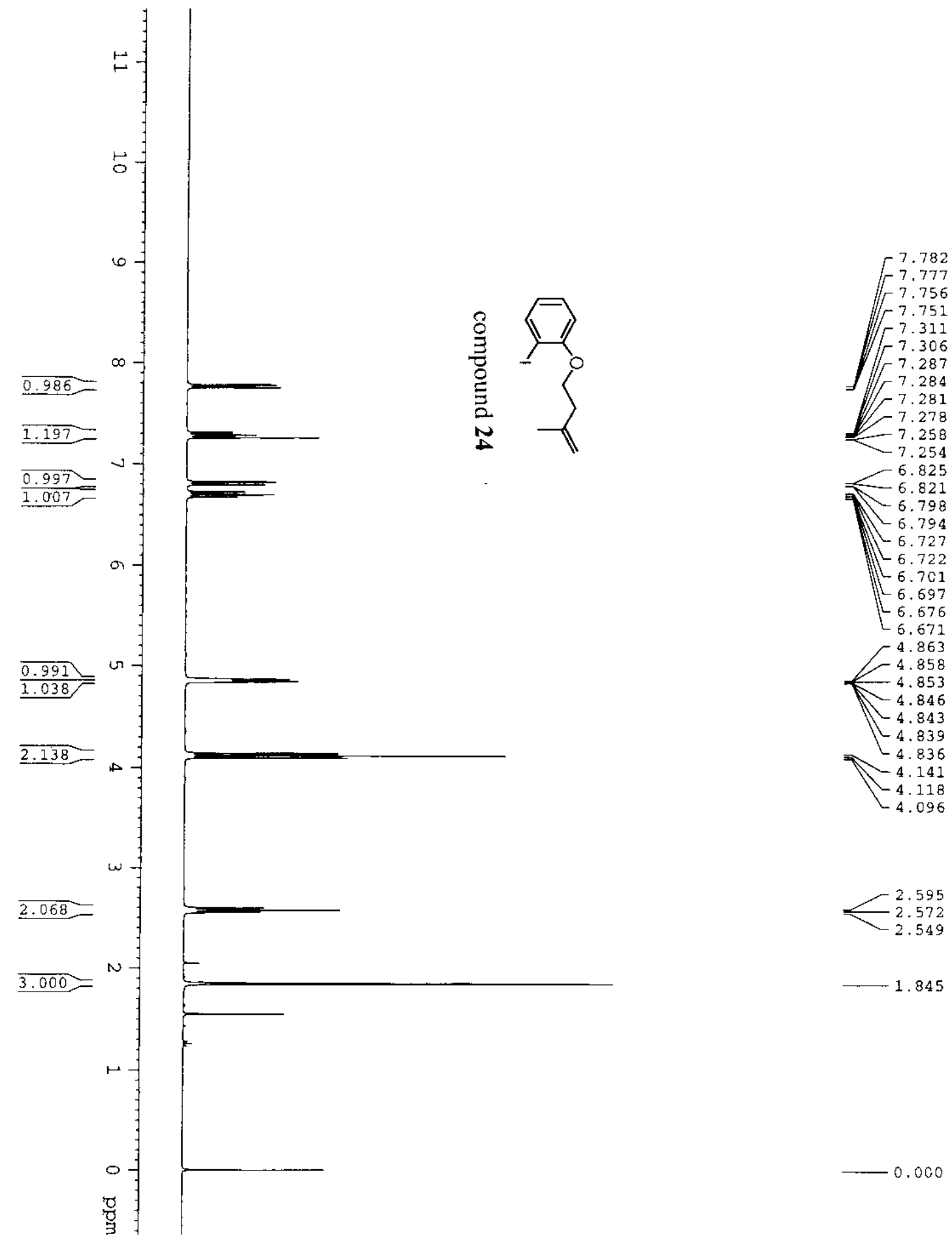

$-0.000$ 

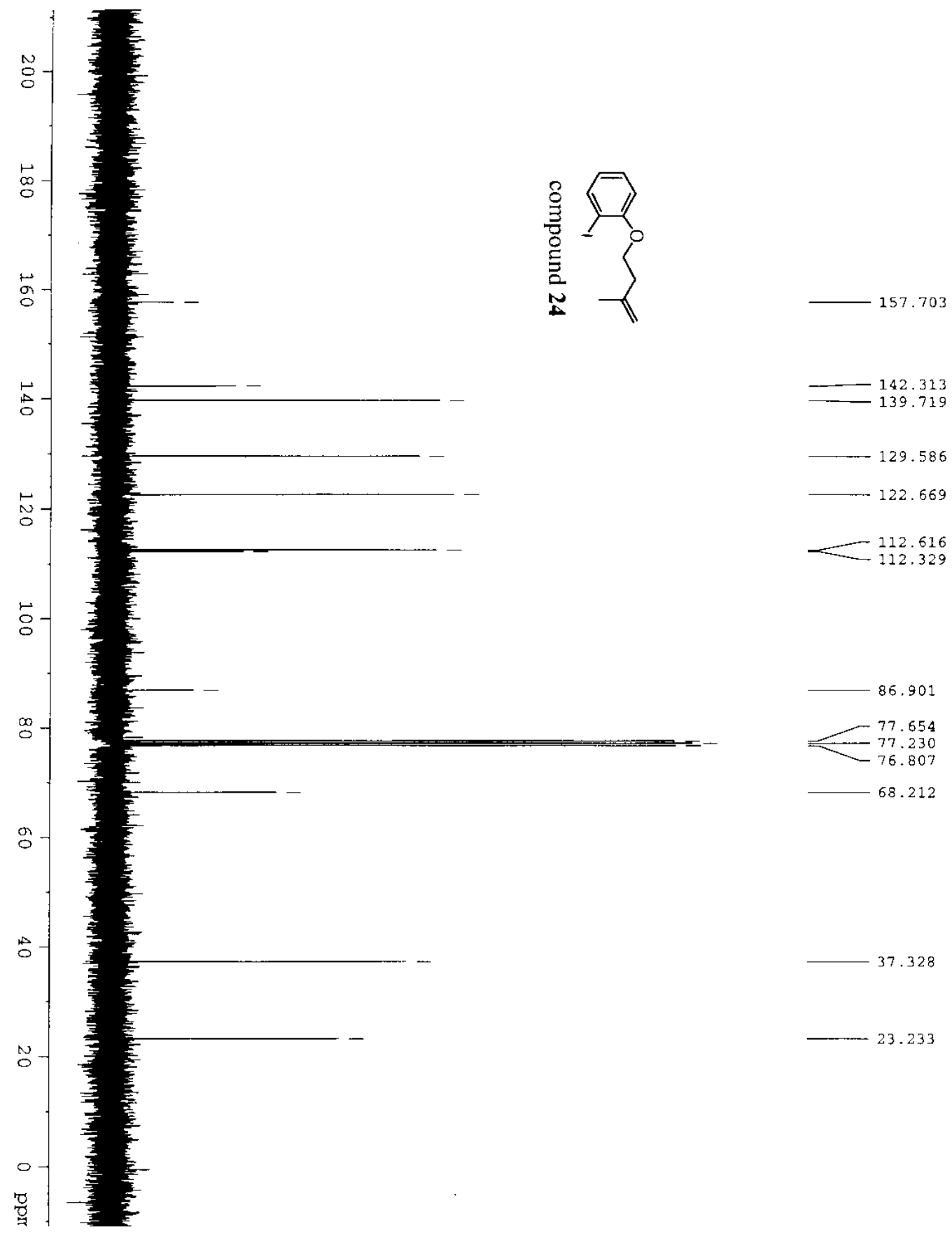

-112.616
$\square$
$\square$

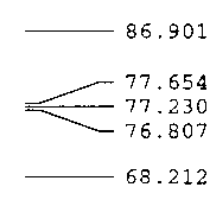

$-37.328$

$-23.233$ 


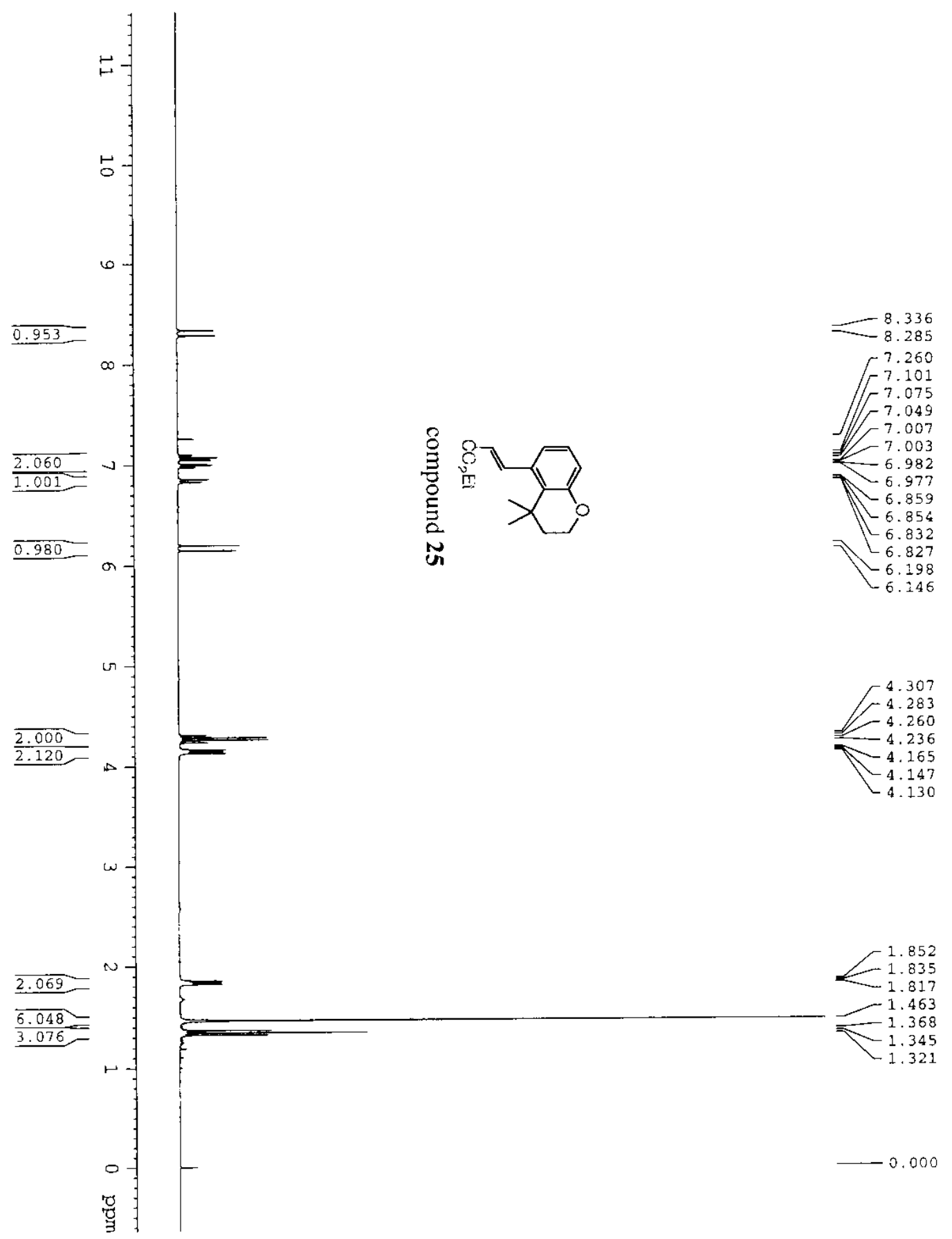




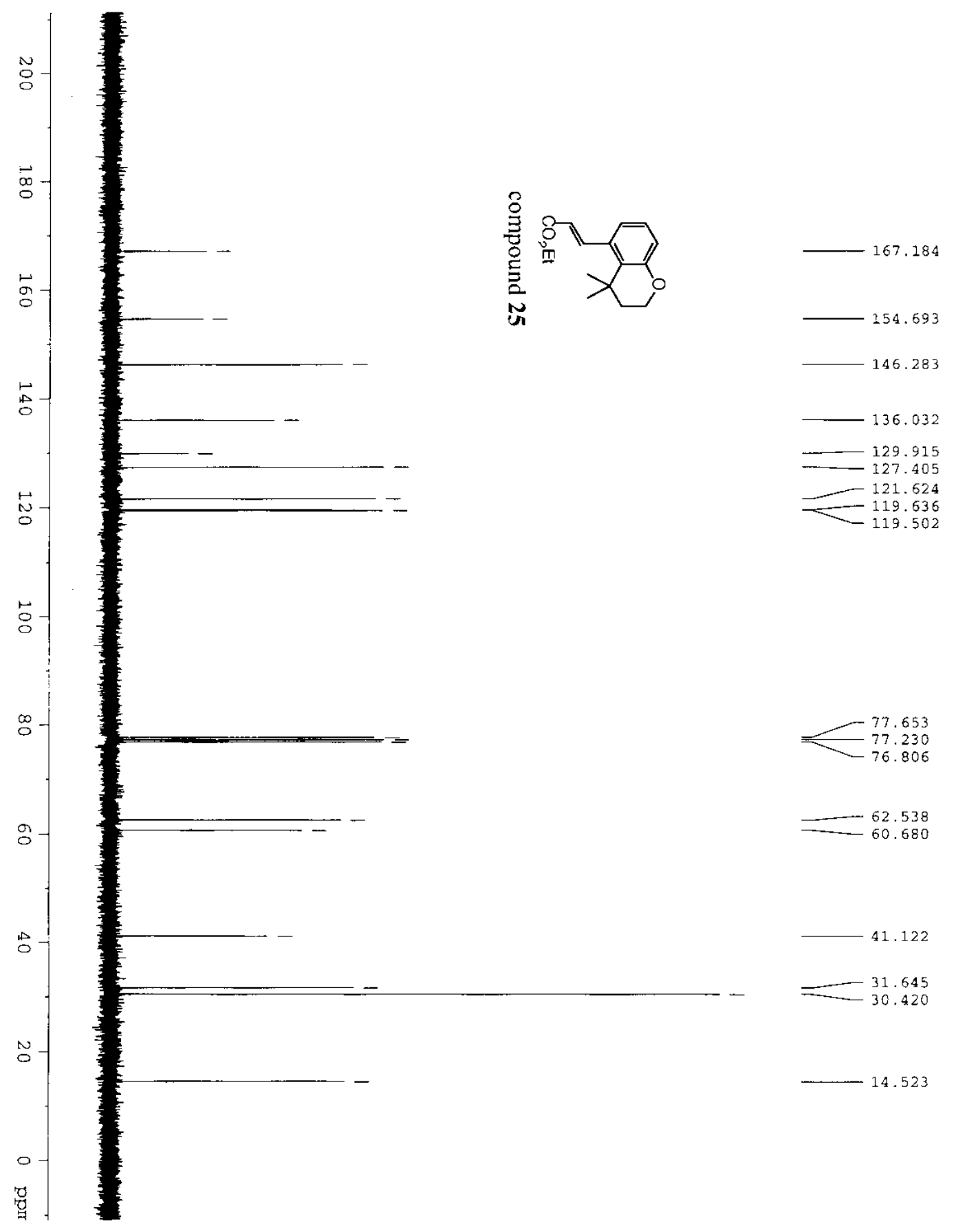




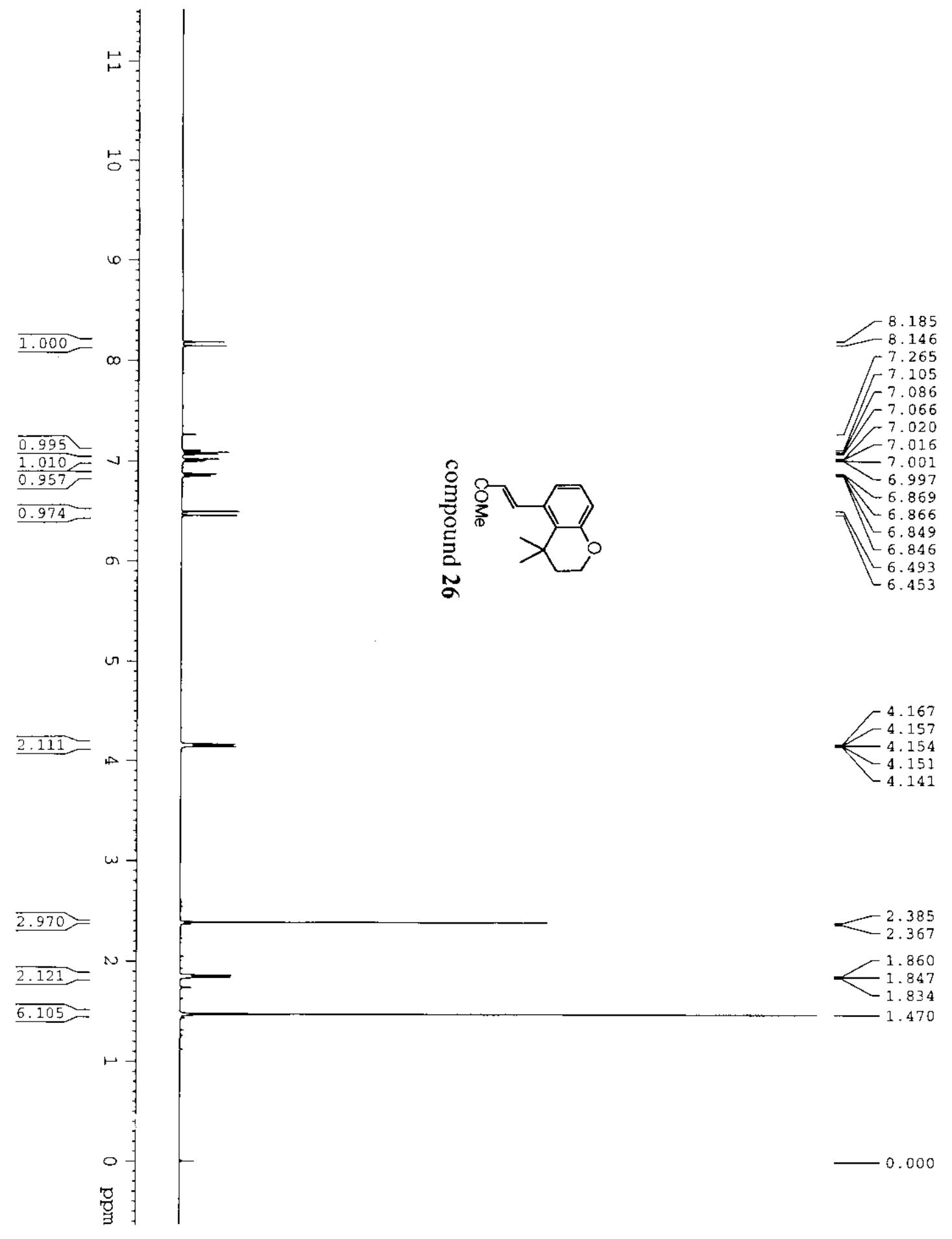



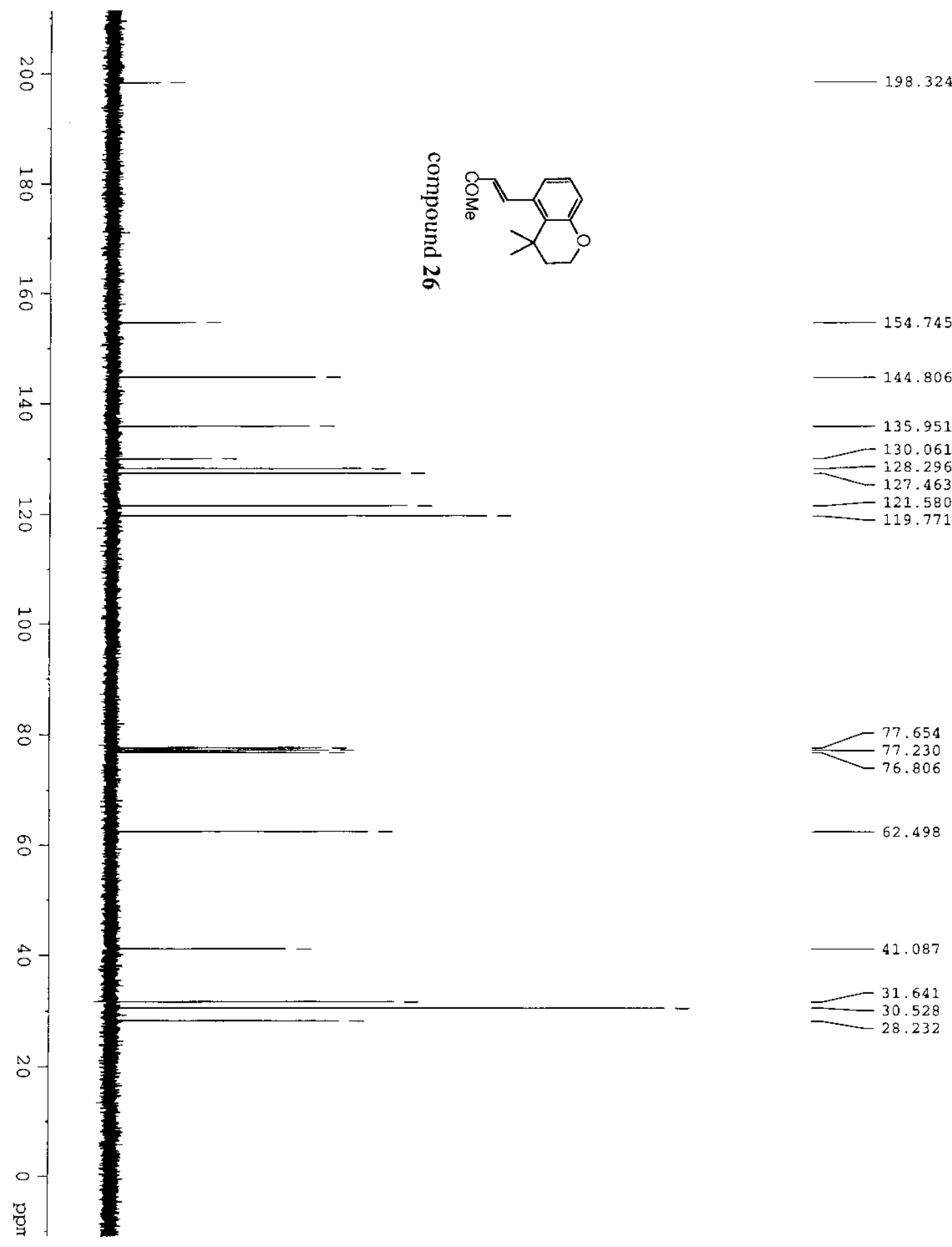

$-144.806$
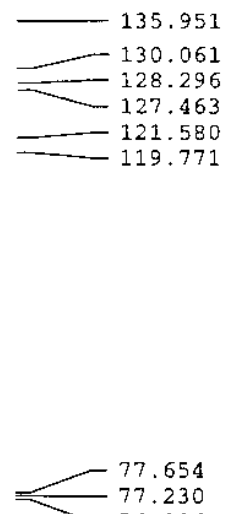

$-76.806$

62.498

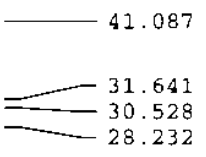

